SLAC-TN-04-018

SLAC-PROPOSAL-E-166(bis)

Revision Date: June 27, 2003

\title{
Undulator-Based Production of Polarized Positrons
}

\section{A Proposal for the 50-GeV Beam in the FFTB}

Gideon Alexander ${ }^{D E T A}$, Perry Anthony ${ }^{S L}$, Vinod Bharadwaj ${ }^{S L}$, Yuri K. Batygin ${ }^{S L}$, Ties Behnke ${ }^{D E, S L}$, Steve Berridge ${ }^{U T}$, Gary R. Bower ${ }^{S L}$, William Bugg ${ }^{U T}$, Roger $\operatorname{Carr}^{S L}$, Eugene Chudakov $^{J L}$, James E. Clendenin ${ }^{S L}$, Franz-Josef Decker ${ }^{S L}$, Yuri Efremenko ${ }^{U T}$,

Ted Fieguth $^{S L}$, Klaus Flöttmann ${ }^{D E}$, Masafumi Fukuda ${ }^{T O}$, Vahagn Gharibyan ${ }^{D E}$, Thomas Handler $^{U T}$, Tachishige Hirose ${ }^{W A}$, Richard H. Iverson ${ }^{S L}$, Yuri Kamychkov ${ }^{U T}$, Hermann Kolanoski ${ }^{H U}$, Thomas Lohse ${ }^{H U}$, Changguo Lu ${ }^{P R}$, Kirk T. McDonald ${ }^{P R, 1}$ Norbert Meyners $^{D E}$, Robert Michaels ${ }^{J L}$, Alexandre A. Mikhailichenko ${ }^{C O}$, Klaus Mönig ${ }^{D E}$, Gudrid Moortgat-Pick ${ }^{D U}$, Michael Olson ${ }^{S L}$, Tsunehiko Omori ${ }^{K E}$, Dimitry Onoprienko ${ }^{B R}$, Nikolaj Pavel ${ }^{H U}$, Rainer Pitthan ${ }^{S L}$, Milind Purohit ${ }^{S C}$, Louis Rinolfi ${ }^{C E}$,

K.-Peter Schüler ${ }^{D E}$, John C. Sheppard ${ }^{S L, 1}{ }^{S t e f a n}$ Spanier $^{U T}$, Achim Stahl ${ }^{D E}$, Zen M. Szalata ${ }^{S L}$, James Turner ${ }^{S L}$, Dieter Walz ${ }^{S L}$, Achim Weidemann ${ }^{S C}$, John Weisend ${ }^{S L}$

${ }^{B R}$ Brunel University, Uxbridge, Middlesex UB8 3PH, United Kingdom CE CERN, CH-1211 Geneva 23, Switzerland $C O$ Cornell University, Ithaca, NY 14853 DE DESY, D-22603 Hamburg, Germany

$D U$ University of Durham, Durham DH1 3HP, United Kingdom

$J L$ Thomas Jefferson National Accelerator Facility, Newport News, VA 23606

$H U$ Humboldt University, Berlin, Germany KE KEK, Tsukuba-shi, Ibaraki, Japan

$P R$ Joseph Henry Laboratory, Princeton University, Princeton, NJ 08544

${ }^{S C}$ University of South Carolina, Columbia, SC 29208 SL SLAC, Stanford, CA 94309

${ }^{T A}$ University of Tel Aviv, Tel Aviv 69978, Israel

TO Tokyo Metropolitan University, Hachioji-shi, Tokyo, Japan

UT University of Tennessee, Knoxville, TN 37996

WA Waseda University, 389-5 Shimooyamada-machi,Machida,Tokyo 194-0202

Updates of this living document are available here:

http://www-project.slac.stanford.edu/lc/local/PolarizedPositrons/E-166bis.pdf

The E-166 Project Website:

http://www-project.slac.stanford.edu/lc/local/PolarizedPositrons/index.htm

$1 \quad$ Co-Spokesperson

Work supported in part by the Department of Energy contract DE-AC03-76SF00515. 


\section{Executive Summary}

The full exploitation of the physics potential of future linear colliders such as the JLC, NLC, and TESLA will require the development of polarized positron beams. In the proposed scheme of Balakin and Mikhailichenko [1] a helical undulator is employed to generate photons of several $\mathrm{MeV}$ with circular polarization which are then converted in a relatively thin target to generate longitudinally polarized positrons.

This experiment, E-166, proposes to test this scheme to determine whether such a technique can produce polarized positron beams of sufficient quality for use in future linear colliders. The experiment will install a meter-long, short-period, pulsed helical undulator in the Final Focus Test Beam (FFTB) at SLAC. A low-emittance 50-GeV electron beam passing through this undulator will generate circularly polarized photons with energies up to $10 \mathrm{MeV}$. These polarized photons are then converted to polarized positrons via pair production in thin targets. Titanium and tungsten targets, which are both candidates for use in linear colliders, will be tested. The experiment will measure the flux and polarization of the undulator photons, and the spectrum and polarization of the positrons produced in the conversion target, and compare the measurement results to simulations. Thus the proposed experiment directly tests for the first time the validity of the simulation programs used for the physics of polarized pair production in finite matter, in particular the effects of multiple scattering on polarization.

Successful comparison of the experimental results to the simulations will lead to greater confidence in the proposed designs of polarized positrons sources for the next generation of linear colliders.

This experiment requests six-weeks of time in the FFTB beam line: three weeks for installation and setup and three weeks of beam for data taking. A 50-GeV beam with about twice the SLC emittance at a repetition rate of $30 \mathrm{~Hz}$ is required. 


\section{Contents}

1 Introduction $\quad 2$

1.1 Undulator Based Production of Polarized Positrons . . . . . . . . . . . . . 2

1.2 The Need for a Demonstration Experiment . . . . . . . . . . . . . . . 3

1.3 Comparison with Positron Sources for Linear Colliders . . . . . . . . . . . . 4

1.4 Physics Opportunities at a Linear Collider with Polarized Electrons and Polarized Positrons ........................ . . . 4

1.4.1 Effective Polarization . . . . . . . . . . . . . . . . . . 6

1.4.2 Standard Model Physics . . . . . . . . . . . . . . . . . 7

1.4.3 Enhancement of Effective Luminosity . . . . . . . . . . . . . . . 7

1.4.4 Physics beyond the Standard Model . . . . . . . . . . . . . . . 8

1.4.5 Transversely Polarized Beams . . . . . . . . . . . . . . . . . 9

1.4.6 Precision Measurement of Beam Polarization . . . . . . . . . . . . . . 10

1.4.7 Summary of the Physics Potential . . . . . . . . . . . . . . 11

2 Principles of the Experiment 12

2.1 Overview . . . . . . . . . . . . . . . . . . 12

2.2 Production of Circularly Polarized $\gamma$-Rays . . . . . . . . . . . . . . 12

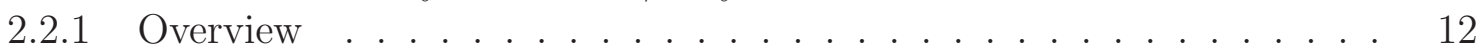

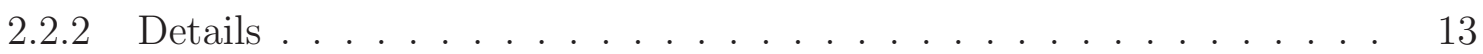

2.3 Production of Polarized Positrons . . . . . . . . . . . . . . . . . . . . 14

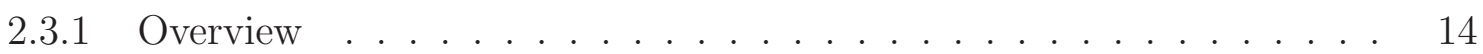

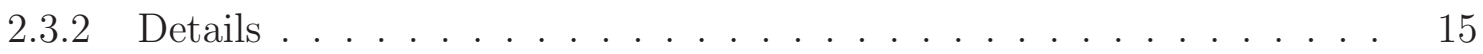

2.4 Polarimetry of $\mathrm{MeV} \gamma$-Rays . . . . . . . . . . . . . . . . . . . . . 18

2.5 Polarimetry of $\mathrm{MeV}$ Positrons . . . . . . . . . . . . . . . . 21

3 The Apparatus $\quad 24$

3.1 Overview . . . . . . . . . . . . . . . . . . . . . 24

3.2 The Beamline . . . . . . . . . . . . . . . . . . 26

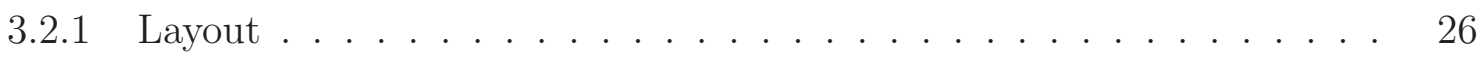

3.2.2 Beam Parameters . . . . . . . . . . . . . . . . . 26

3.2.3 Synchrotron Radiation Background . . . . . . . . . . . . . . . . 28

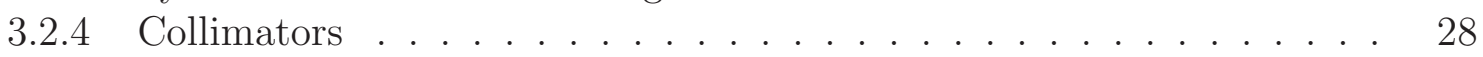

3.2.5 Alignment . . . . . . . . . . . . . . . . . . . . . . . . . . . . . . . . . . . . . . .

3.2 .6 Instrumentation . . . . . . . . . . . . . . . . . . . . . . 30

3.3 The Undulator . . . . . . . . . . . . . . . . . . . . . 30

3.4 The Photon Polarimeter . . . . . . . . . . . . . . . . . . 31

3.4.1 Magnetized Iron Absorber . . . . . . . . . . . . . . . . . 33

3.4.2 Silicon-Tungsten Calorimeter . . . . . . . . . . . . . . . . . . 33

3.4.3 Aerogel Flux Counters . . . . . . . . . . . . . . . . . . . . 34

3.5 The Positron Production Target . . . . . . . . . . . . . . . . 35

3.6 The Positron Polarimeter . . . . . . . . . . . . . . . . . 36

3.6.1 The Positron Transport System . . . . . . . . . . . . . . . . 37

3.6.2 The Magnetized Iron Absorber . . . . . . . . . . . . . . . . . . 38 
3.6.3 The CsI Calorimeter . . . . . . . . . . . . . . . . . . . 40

3.7 Data-Acquisition System . . . . . . . . . . . . . . . . . . . . . . 41

4 Measurements of Photon and Positron Polarization 44

4.1 Undulator Photons . . . . . . . . . . . . . . . . . . . . . . . . 44

$4.1 .1 \quad$ Flux . . . . . . . . . . . . . . . . . . . . . . . . . 44

4.1 .2 Polarization . . . . . . . . . . . . . . . . . . 44

4.2 Positrons . . . . . . . . . . . . . . . . . . . . . . . . . . . . . . . 47

$4.2 .1 \quad$ Flux . . . . . . . . . . . . . . . . . . . . . . . . 47

4.2 .2 Polarization . . . . . . . . . . . . . . . . . . . . . 48

5 Experimental Measurements and Setup 51

5.1 Overview . . . . . . . . . . . . . . . . . . . . 51

5.2 Experimental Measurements . . . . . . . . . . . . . . . . . . . 51

5.3 Experiment Setup . . . . . . . . . . . . . . . . . . . . 52

5.3.1 Status of Required Beamline Equipment . . . . . . . . . . . 52

5.3.2 Installation and Check Out Requirements . . . . . . . . . . . . 53

5.3.3 Beam Tuning and System Integration . . . . . . . . . . . . . . 57

6 References $\quad 58$ 


\section{List of Figures}

1 Conceptual scheme for undulator-based production of polarized positrons. . 3

2 Conceptual layout of the experiment. . . . . . . . . . . . . . . . 4

3 Effective polarization vs.positron polarization. . . . . . . . . . . . 7

4 Selectron pair production with longitudinally polarized beams. . . . . . . 9

5 Search for extra dimension with transversely polarized beams. . . . . . . . . 10

6 Photon number spectrum and helicity spectrum. . . . . . . . . . . . . . . 14

7 Photon number spectrum and helicity spectrum. . . . . . . . . . . . . 15

8 Longitudinal polarization of positrons from a circularly polarized photon. $\quad 16$

9 Longitudinal polarization of positrons from a thin target. . . . . . . . . 16

10 Positron energy and polarization. . . . . . . . . . . . . . . . 17

11 Positron energy spectrum for Ti targets. . . . . . . . . . . . . . . 18

12 Concept of transmission polarimetry. . . . . . . . . . . . . . . . . . . 18

13 Compton scattering cross sections. . . . . . . . . . . . . . . 19

14 Photon attenuation length and transmission in iron. . . . . . . . . . . . 20

15 Figure of merit for transmission polarimetry. . . . . . . . . . . . . . . . . 21

16 Polarization transfer from positrons to photons. . . . . . . . . . . . . . . 22

17 Analyzing power for positron polarimetry. . . . . . . . . . . . . . . 23

18 Conceptual layout of the experiment. . . . . . . . . . . . . . . . . . 24

19 Conceptual layout of positron production and polarimetry. . . . . . . . . . 25

20 Prototype of the helical undulator. . . . . . . . . . . . . . . . 31

21 Schematic representation of the undulator with pulsing circuit. . . . . . . . 31

22 Mechanical drawing of the undulator. . . . . . . . . . . . . . . . . . 32

23 Photon transmission probability through $15 \mathrm{~cm}$ of iron . . . . . . . . . 33

24 The silicon tungsten calorimeter. . . . . . . . . . . . . . . . . 34

25 The aerogel flux counter. . . . . . . . . . . . . . . . 35

26 Layout of the positron polarimeter. . . . . . . . . . . . . . . . . . 37

27 Momentum acceptance of the positron polarimeter. . . . . . . . . . . 38

28 Photons in the upstream part of the positron polarimeter. . . . . . . . . . 39

29 Positrons in the upstream part of the positron polarimeter. . . . . . . . . . 39

30 Probability that a positron reaches the reconversion target. . . . . . . . . . 40

31 Probability that a background photon reaches the CsI array. . . . . . . . . . 40

32 Field map along the axis of the magnetized iron absorber. . . . . . . . . . . 41

33 CsI readout electronics. . . . . . . . . . . . . . . . . . . . 42

34 Photon spectra after the iron absorber. . . . . . . . . . . . . . . . . . . . . . . . . . . . . . . . 45

35 Analyzing power vs. reconversion target thickness. . . . . . . . . . . . . . 48

36 Analyzing power vs. momentum acceptance. . . . . . . . . . . . . . . . 49

\section{List of Tables}

1 TESLA, NLC, E-166 polarized positron parameters. . . . . . . . . . 5

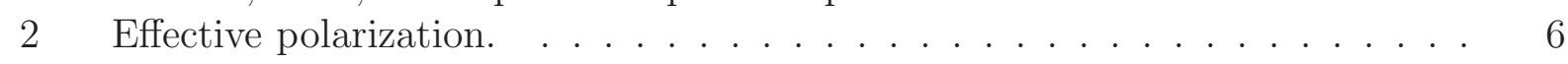

3 Scaling factors for $W W$ and $Z Z$ production. . . . . . . . . . . . 8 
4 Fraction of colliding particles for different beam polarizations. . . . . . . 8

$5 \quad$ E-166 beam parameters. . . . . . . . . . . . . . . 26

$6 \quad$ E-166 photon beam parameters. . . . . . . . . . . . . . . 27

7 Photon flux from the helical undulator. . . . . . . . . . . . . . . . . . . . . . 29

8 Parameters of the helical undulator system. . . . . . . . . . . . . . . 32

9 Positron yields from Ti and W-Re targets. . . . . . . . . . . . . . . . . 36

10 Parameters of the positron polarimeter. . . . . . . . . . . . . . 37

11 Data-acquisition system. . . . . . . . . . . . . . . . . 43

12 Photon polarization measurements using the aerogel detector. . . . . . . . . 47

13 Simulation results: positron energy scan. . . . . . . . . . . . . . . 50

14 E-166 beam parameter request. . . . . . . . . . . . . . . . . 51

15 Status of E-166 beamline equipment. . . . . . . . . . . . . . . 54

16 Photon and positron polarimetry devices and detectors. . . . . . . . . . 55

17 Detector installation and test plan. . . . . . . . . . . . . . 56 


\section{Introduction}

The full exploitation of the physics potential of future linear colliders such as the JLC, NLC, and TESLA will require the development of polarized positron beams.

In the proposed scheme of Balakin and Mikhailichenko [1] a helical undulator is employed to generate photons of several $\mathrm{MeV}$ with circular polarization which are then converted in a relatively thin target to generate longitudinally polarized positrons.

To advance progress in this field, this experiment, E-166, proposes to test this scheme to determine whether such a technique can produce polarized positron beams of sufficient quality for use in future Linear Colliders. The experiment will install a 1-meter-long, shortperiod $\left(\lambda_{u}=2.4 \mathrm{~mm}, K=0.17\right)$, pulsed helical undulator in the Final Focus Test Beam (FFTB) at SLAC. A low-emittance 50-GeV electron beam passing through this undulator will generate circularly polarized photons with energies up to a cutoff energy of about 10 $\mathrm{MeV}$. These polarized photons are then converted to polarized positrons via pair production in thin targets.

This section describes the concept of polarized positron production, the need for experimental verification, and some of the more important physics justifications for the need for polarized positrons in Linear Colliders.

\subsection{Undulator Based Production of Polarized Positrons}

A polarized positron source for a Linear Collider was first proposed by Balakin and Mikhaili-chenko in 1979 in the framework of the VLEPP project [1]. The concept, schematically sketched in Fig. 1, sends the high energy ( $\geq 150 \mathrm{GeV})$ electron beam of a Linear Collider through a $(\sim 200$ m-long $)$ helical undulator to produce circularly polarized photons with energies of about $11 \mathrm{MeV} .^{1}$ While the electrons are further accelerated and brought into collision after passing through the undulator, the photons are converted in a thin target into electron-positron pairs. Here the polarization state of the photons is transferred to the positrons and electrons (see below for details). Only the on-axis photons of the helical undulator radiation are completely circularly polarized; the degree of polarization is decreasing with increasing emission angle. Hence, the polarization of the photons and of the generated positrons can be increased at the expense of the total number of positrons by collimation. The positrons are captured behind the target similarly to the case of a conventional positron source $[2,3]$, and fed into a linac.

This undulator-based positron source concept offers the additional advantage that the heat load on the target is less than that of a conventional source, and so the former is very well suited for the production of high intensity positron beams [4]. An undulator-based polarized positron sources can in principle be realized independently of the linac technology, i.e., independently of the details of the required pulse structure, because the number of produced positrons scales with the number of the electrons in the drive linac, and the pulse

\footnotetext{
${ }^{1}$ Alternatively, the undulator could be placed in the electron beam beyond the $e^{+} e^{-}$interaction point, using the "spent" electron beam. The beam quality of the disrupted electron beam is, however, poor due to the strong beam-beam interaction (beamstrahlung). Moreover, the electron beam quality, and hence the positron production efficiency, depend on the details of the collision (offsets etc.), which makes this option even more problematic.
} 


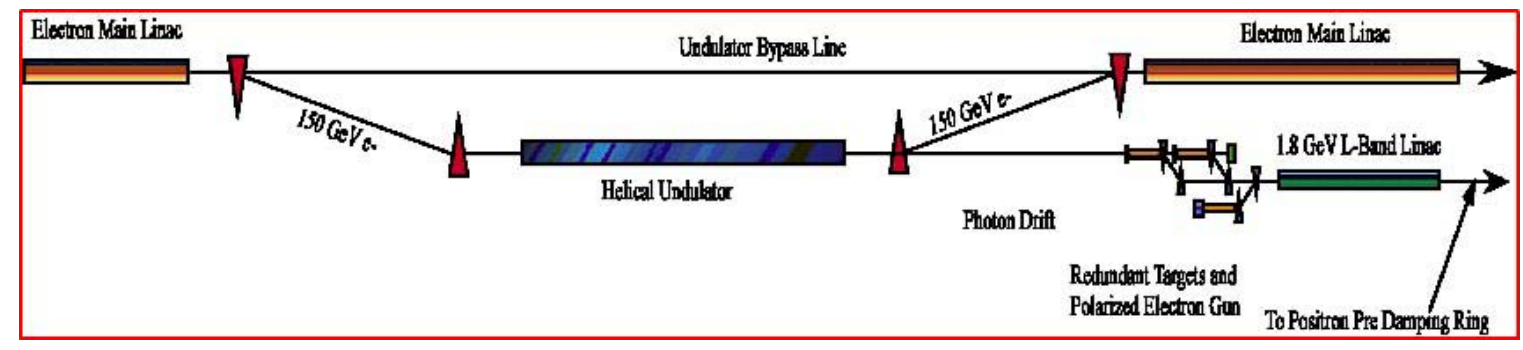

Figure 1: Conceptual scheme for undulator-based production of polarized positrons.

structure of the electrons is directly copied to that of the positrons. In this sense it is an option for all Linear Collider projects.

A related approach for the production of polarized positrons is to create circularly polarized photons by means of Compton backscattering of laser light off a high-intensity electron beam $[5,6,7]$. Undulator radiation can be thought of as Compton backscattering of the virtual photons of the undulator, and hence the photon spectrum and the polarization characteristics of Compton backscattered photons are very similar to those of undulator radiation. The requirements for the pulsed laser system to implement positron production are extremely demanding, so the use of the electron beam plus undulator offers significant technical advantages compared to Compton backscattering of real photons.

\subsection{The Need for a Demonstration Experiment}

The aim of the proposed experiment E-166 is to test the fundamental process of polarization transfer in an electromagnetic cascade. For this, a simplified version of the scheme shown in Fig. 1 will be used, in which a 50-GeV electron beam passes through a 1-m-long undulator as shown in Fig. 2. The resulting photon beam of MeV energy is converted to positrons (and electrons) in a thin target, after which the polarization of the positrons (and photons) is analyzed.

While the basic cross sections for the QED processes relevant to polarization transfer were derived in the late 1950's, experimental verification of the polarization development in an electromagnetic cascade is still missing. From this point of view, the proposed experiment has some general scientific aspects in addition to its importance for Linear Colliders.

Each approximation in the modeling of electromagnetic cascades seems to be well justified in itself, but the complexity of polarization transfer in cascades makes the comparison with an experiment desirable, so that the decision whether a Linear Collider should be built with or without a polarized positron source can be based on solid grounds. The achievable precision of the proposed transmission polarimetry of $5-10 \%$ is sufficient for this purpose. This experiment, however, will not address detailed systems issues related to polarized positron production for a Collider, such as capture efficiency, target thermal hydrodynamics, radiation damage in the target, or an undulator prototype suitable for use at a Linear Collider; such issues are well within the scope of $R \& D$ by a Linear Collider project that chooses to implement a polarized positron source based on a helical undulator. 


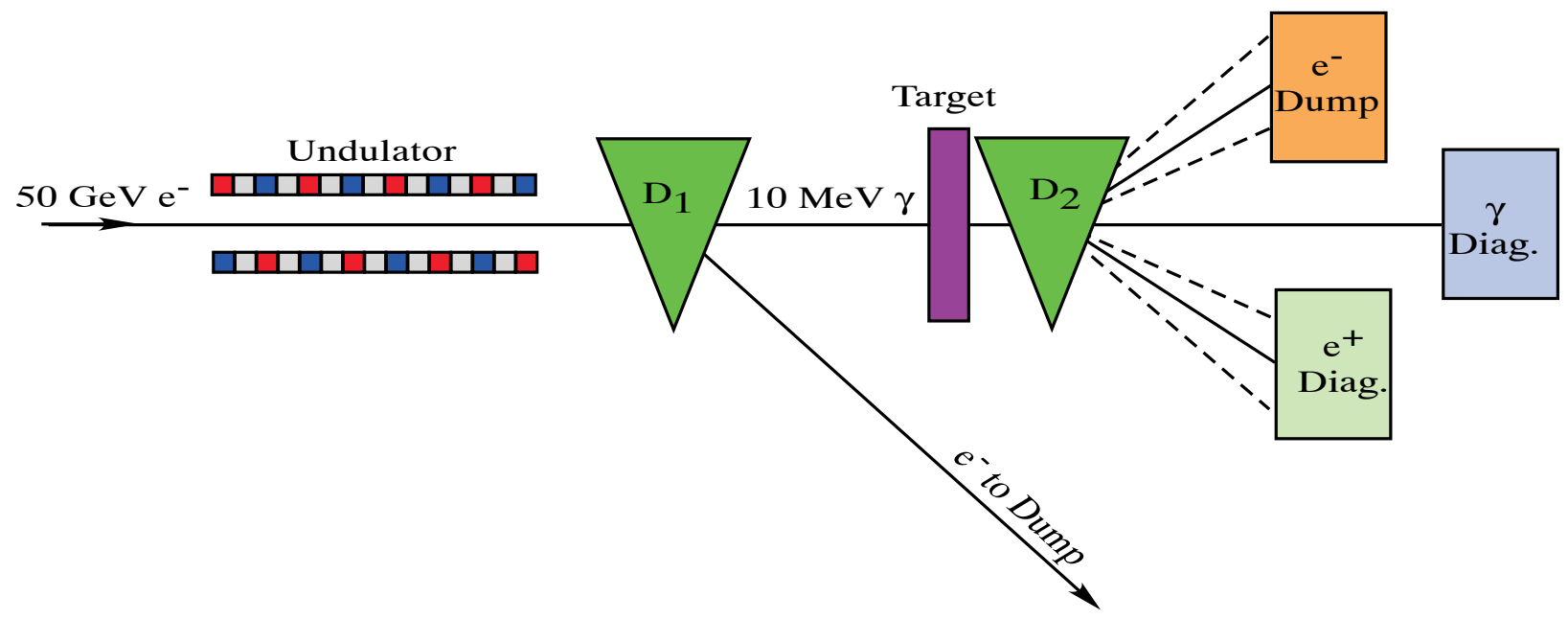

Figure 2: Conceptual layout (not to scale) of the experiment to demonstrate the production of polarized positrons in the SLAC FFTB. 50-GeV electrons enter from the left and pass through an undulator to produce a beam of circularly polarized photons of MeV energy. The electrons are deflected away from the photons by the $\mathrm{D}_{1}$ magnet. The photons are converted to electrons and positrons in a thin target. The polarization of the positrons, and of the photons, are measured in polarimeters based on Compton scattering of electrons in magnetized iron.

\subsection{Comparison with Positron Sources for Linear Colliders}

Table 1 shows a comparison between the parameters of the TESLA positron source system [8], the NLC undulator-based positron source option [9], and those of the proposed experiment. The TESLA baseline design uses a planar undulator for unpolarized positron production; the NLC design and FFTB experiment use helical undulators. As seen in Table 1 , the characteristic photon energy, $E_{c 10}$, for E-166 is very similar to that of both TESLA and NLC. Thus, the positron yield and polarization are also similar to what is expected in the Linear Collider designs. This is accomplished with the use of a much lower energy electron beam by decreasing the undulator period appropriately.

\subsection{Physics Opportunities at a Linear Collider with Polarized Electrons and Polarized Positrons}

Polarized electrons have been a part of each of the different Linear Collider proposals for a long time. Recently much scrutiny has been given to the case for polarized positrons in addition to polarized electrons. A consensus has emerged that polarized positrons are a highly desirable option for a Linear Collider.

The importance of beam polarization in general was demonstrated e.g., at the SLAC Linear Collider (SLC). Because of the high degree of electron polarization ( during its last run in 1997/98, an average longitudinal beam polarization $P_{e^{-}}=74 \%$ was reached [10]) one 
Table 1: Parameters of polarized positrons beams at TESLA, NLC and the present experiment.

\begin{tabular}{|c|c|c|c|c|}
\hline Parameter & Units & TESLA* & NLC & FFTB \\
\hline Beam energy $E_{e}$ & $\mathrm{GeV}$ & $150-250$ & 150 & 50 \\
\hline$N_{e} /$ bunch & - & $3 \times 10^{10}$ & $8 \times 10^{9}$ & $1 \times 10^{10}$ \\
\hline$N_{\text {bunch }} /$ pulse & - & 2820 & 190 & 1 \\
\hline Pulses/s & $\mathrm{Hz}$ & 5 & 120 & 30 \\
\hline Undulator type & - & planar & helical & helical \\
\hline Undulator strength $K$ & - & 1 & 1 & 0.17 \\
\hline Undulator period $\lambda_{u}$ & $\mathrm{~cm}$ & 1.4 & 1.0 & 0.24 \\
\hline $1^{s t}$ Harmonic cutoff, $E_{\mathrm{c} 10}$ & $\mathrm{Mev}$ & $9-25$ & 11 & 9.6 \\
\hline$d N_{\gamma} / d L$ & photons $/ \mathrm{m} / e^{-}$ & 1 & 2.6 & 0.37 \\
\hline Undulator length $L$ & $\mathrm{~m}$ & 135 & $132^{\dagger}$ & 1 \\
\hline Target material & - & Ti-alloy & Ti-alloy & Ti-alloy \\
\hline Target thickness & rad. len.' & 0.4 & 0.5 & 0.5 \\
\hline Yield & $e^{+} /$photon $(\%)$ & $1-5$ & $1.5^{\ddagger}$ & 0.5 \\
\hline Capture efficiency & $\%$ & 25 & 20 & - \\
\hline$N_{+} /$pulse & - & $8.5 \times 10^{12}$ & $1.5 \times 10^{12}$ & $2 \times 10^{7}$ \\
\hline$N_{+} /$bunch & - & $3 \times 10^{10}$ & $8 \times 10^{9}$ & $2 \times 10^{7}$ \\
\hline Polarization $P^{+}$ & $\%$ & - & $40-70$ & $40-70$ \\
\hline
\end{tabular}

* TESLA baseline design; TESLA polarized $e^{+}$parameters (undulator and polarization) are the same as for the NLC.

$\dagger$ A length of $132 \mathrm{~m}$ is required for a unity gain $e^{-} \rightarrow e^{+}$system. An undulator length of $200 \mathrm{~m}$ is under consideration in order to provide $50 \%$ overhead in positron production.

$\ddagger$ Includes the effect of photon collimation at $\gamma \theta_{\text {cut }}=1.414$. 
of the world's most precise measurements of the weak mixing angle at $Z$-pole energies was performed.

Having both beams polarized offers a number of advantages:

- Higher effective polarization.

- Increased signal to background in studies of Standard Model Physics.

- Enhancement of the effective luminosity.

- Precise analysis of many kinds of non-standard couplings.

- The option to use transversely polarized beams.

- Improved accuracy in measuring the polarization.

Details and examples of these effects are summarized in [13] and some key points are highlighted here.

\subsubsection{Effective Polarization}

If both electron and positron polarization are available it is often useful to express the polarization in terms of an effective polarization, $P_{\text {eff }}$. As an example in the study of the left-right asymmetry of s-channel vector particle exchange the effective polarization is defined as

$$
P_{\text {eff }}=\frac{P_{e^{-}}-P_{e^{+}}}{1-P_{e^{-}} P_{e^{+}}} .
$$

The error of the measurement of the left-right asymmetry scales roughly with $1-P_{\text {eff }}$, favoring a high effective polarization. The final error on the asymmetries measured will in many cases be limited by the error on the polarization. Having both beams polarized significantly decreases the error on the effective polarization. This is illustrated in Fig. 3 and in Table 2.

Table 2: The effective polarization (1) for various $e^{-}$and $e^{+}$polarizations.

\begin{tabular}{|l|c|c|c|c|c|c|}
\hline \hline & \multicolumn{3}{|c|}{$P_{e^{-}}= \pm 0.8$} & \multicolumn{3}{|c|}{$P_{e^{-}}= \pm 0.9$} \\
\hline \hline$P_{e^{+}}$ & 0 & $\mp 0.4$ & $\mp 0.6$ & 0 & $\mp 0.4$ & $\mp 0.6$ \\
\hline$\left|P_{\text {eff }}\right|$ & 0.80 & 0.91 & 0.95 & 0.90 & 0.95 & 0.97 \\
\hline $1-\left|P_{\text {eff }}\right|$ & 0.20 & 0.09 & 0.05 & 0.10 & 0.05 & 0.03 \\
\hline \hline
\end{tabular}




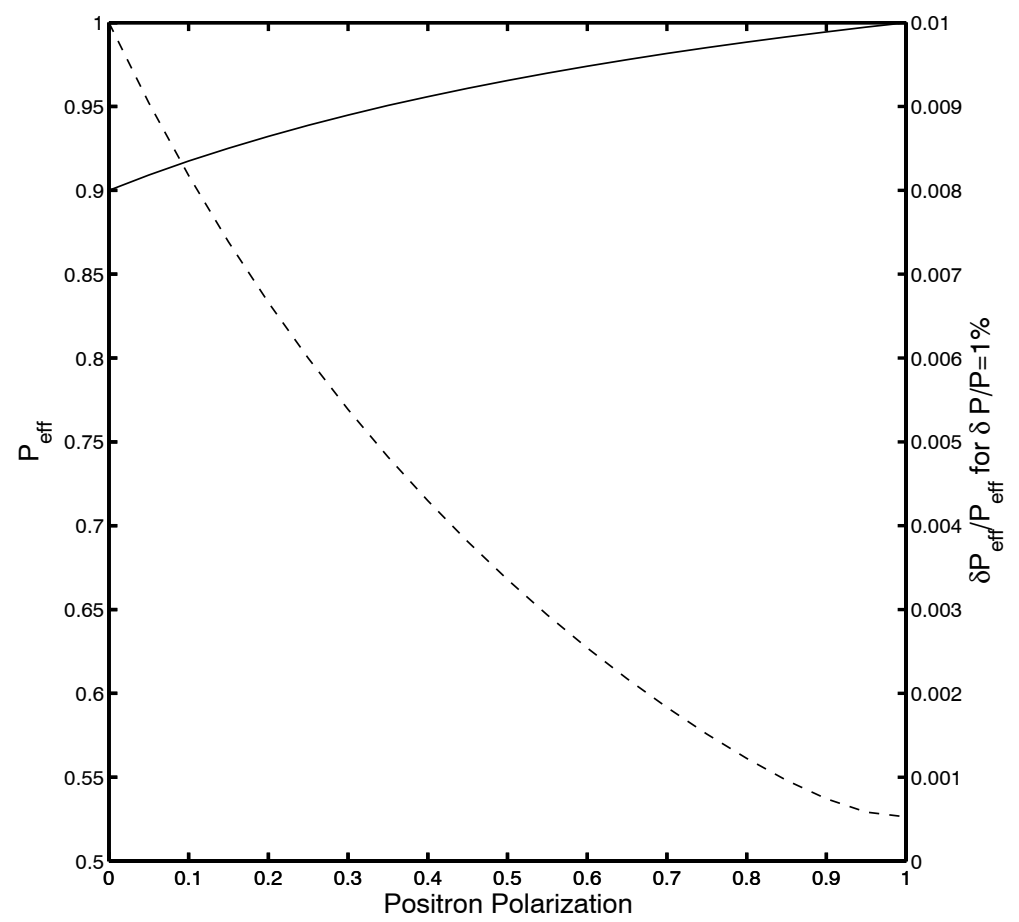

Figure 3: Solid curve: the effective polarization (1) at a Linear Collider as a function of positron polarization, assuming an electron polarization of $90 \%$. Dashed curve: the relative error in the effective polarization. From [11].

\subsubsection{Standard Model Physics}

Standard Model physics based on $W W$ or $Z Z$ production depends on the effective polarization of the two lepton beams. This can be used to either enhance or suppress the standard model processes (see Table 3 for some examples). As the dominant background processes for many new physics searches are $W W$ and $Z Z$ production, suppressing their contributions can enhance the search potential for new physics. A positron polarization of about $P_{e^{+}}=60 \%$ would double the suppression of the $W W$ background. Similarly the background from single $W^{ \pm}$production depends on the polarization of the positron beam, as well as on the electron beam polarization.

\subsubsection{Enhancement of Effective Luminosity}

The chiral structure of Standard Model $s$-channel processes is given by (V-A) couplings. This means that only the $(L R)$ and $(R L)$ configurations of the initial $e^{ \pm}$contribute. The fraction of colliding particles is therefore

$$
\frac{1}{2}\left(1-P_{e^{-}} P_{e^{+}}\right) \equiv \frac{\mathcal{L}_{\text {eff }}}{\mathcal{L}}
$$

which defines an effective luminosity $\mathcal{L}_{\text {eff }}$. If both beams are polarized this can be enhanced, as illustrated in Table 4. 
Table 3: Scaling factors for $W W$ and $Z Z$ production. These mechanisms are the dominant backgrounds for new physics searches, so smaller scaling factors correspond to better background suppression.

\begin{tabular}{|c|c|c|c|}
\hline \hline$P_{e^{-}}$ & $P_{e^{+}}$ & $e^{+} e^{-} \rightarrow W^{+} W^{-}$ & $e^{+} e^{-} \rightarrow Z Z$ \\
\hline \hline 0 & 0 & 1.0 & 1.0 \\
\hline 0.8 & 0 & 0.2 & 0.76 \\
\hline-0.8 & 0 & 1.8 & 1.25 \\
\hline 0.8 & -0.6 & 0.1 & 1.05 \\
\hline-0.8 & 0.6 & 2.85 & 1.91 \\
\hline \hline
\end{tabular}

Table 4: Fraction of colliding particles $\left(\mathcal{L}_{\text {eff }} / \mathcal{L}\right)$ and the effective polarization $\left(P_{\text {eff }}\right)$ for different beam polarization configurations, which are characteristic for $(\mathrm{V}-\mathrm{A})$ processes in the $s$-channel [13].

\begin{tabular}{|c|c|c|c|c|c|c|c|}
\hline \hline$P_{e^{-}}$ & $P_{e^{+}}$ & $\mathrm{RL}$ & $\mathrm{LR}$ & $\mathrm{RR}$ & $\mathrm{LL}$ & $P_{\text {eff }}$ & $\mathcal{L}_{\text {eff }} / \mathcal{L}$ \\
\hline \hline 0 & 0 & 0.25 & 0.25 & 0.25 & 0.25 & 0. & 0.5 \\
\hline-1 & 0 & 0 & 0.5 & 0 & 0.5 & -1 & 0.5 \\
\hline-0.8 & 0 & 0.05 & 0.45 & 0.05 & 0.45 & -0.8 & 0.5 \\
\hline-0.8 & +0.6 & 0.02 & 0.72 & 0.08 & 0.18 & -0.95 & 0.74 \\
\hline \hline
\end{tabular}

\subsubsection{Physics beyond the Standard Model}

Beam polarization is of particular importance for the study of physics beyond the standard model. Supersymmetry (SUSY) is a leading candidate for new physics. However, even the simplest version, the Minimal Supersymmetric Standard Model (MSSM), leads to 105 new free parameters. If SUSY exists, one of the most important studies to be performed will be the determination of the SUSY parameters in as model independent a way as possible and to prove the underlying SUSY assumptions, e.g., that the SUSY particles carry the same quantum numbers (with the exception of the spin) as their Standard Model counterparts.

For example, SUSY transformations associate chiral (anti)fermions to scalars $e_{L, R}^{-} \leftrightarrow \tilde{e}_{L, R}^{-}$ but $e_{L, R}^{+} \leftrightarrow \tilde{e}_{R, L}^{+}$. Both beams have to be polarized in order to prove these associations. [14]. The process $e^{+} e^{-} \rightarrow \tilde{e}^{+} \tilde{e}^{-}$occurs via $\gamma$ and $Z$ exchange in the $s$-channel and via neutralino $\tilde{\chi}_{i}^{0}$ exchange in the $t$-channel. The association can be directly tested only in the $t$-channel 
and the use of polarized beams serves to separate out this channel. Fig. 4 shows an example of this where the selectron masses are close together, $m_{\tilde{e}_{L}}=200 \mathrm{GeV}, m_{\tilde{e}_{R}}=190 \mathrm{GeV}$, so that $\tilde{e}_{L}, \tilde{e}_{R}$ decay via the same decay channels (in this example the other SUSY parameters are taken from the reference scenario SPS1a [15]). The $\tilde{e}_{L}^{-} \tilde{e}_{R}^{+}$pair can be enhanced by the $L L$ configuration of the initial beams. From the Figure, it is seen that just having the electron beam polarized will not help. With $P_{e^{-}}=-80 \%, P_{e^{+}}=0 \%$ the cross section for the combinations $\sigma\left(\tilde{e}_{L}^{-} \tilde{e}_{R}^{+}\right)=102 \mathrm{fb}$ and $\sigma\left(\tilde{e}_{L}^{-} \tilde{e}_{L}^{+}\right)=108 \mathrm{fb}$ are close together. This will essentially not change even if $P_{e^{-}}=-100 \%$ were available.

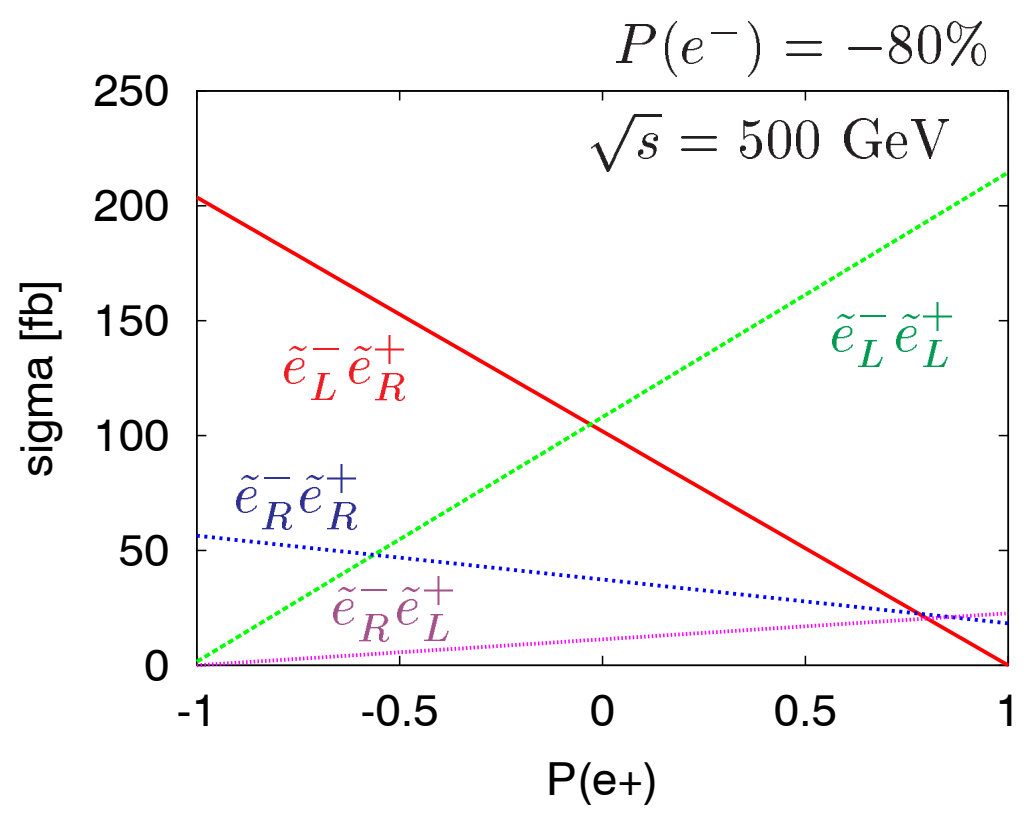

Figure 4: Separation of the selectron pair $\tilde{e}_{L}^{-} \tilde{e}_{R}^{+}$in $e^{+} e^{-} \rightarrow \tilde{e}_{L, R}^{-} \tilde{e}_{L, R}^{+}$with longitudinally polarized beams in order to test the association of chiral quantum numbers to scalar fermions in SUSY transformations [13].

However, if polarized positrons are available, a separation of the different combinations might be possible: $P_{e^{-}}=-80 \%, P_{e^{+}}=-40 \%$ result in $\sigma\left(\tilde{e}_{L}^{-} \tilde{e}_{R}^{+}\right)=143 \mathrm{fb}$ and $\sigma\left(\tilde{e}_{L}^{-} \tilde{e}_{L}^{+}\right)=$ $66 \mathrm{fb}$. With $P_{e^{-}}=-80 \%, P_{e^{+}}=-60 \%, \sigma\left(\tilde{e}_{L}^{-} \tilde{e}_{R}^{+}\right)=163 \mathrm{fb}, \sigma\left(\tilde{e}_{R}^{-} \tilde{e}_{R}^{+}\right)=49 \mathrm{fb}$ and $\sigma\left(\tilde{e}_{L}^{-} \tilde{e}_{L}^{+}\right)=$ $44 \mathrm{fb}$ are obtained ( Fig. 4).

For many SUSY analyzes other SUSY processes are the most important background. Positron polarization can again be used to suppress the undesired process, as illustrated for selectron production in [16].

\subsubsection{Transversely Polarized Beams}

Recently, theoretical interest has increased into the physics opportunities transversely polarized lepton beams offer [17]. The cross section involving transversely polarized leptons is given by

$$
\sigma=\left(1-P_{e^{+}} P_{e^{-}}\right) \sigma_{\mathrm{unpol}}+\left(P_{e^{-}}^{L}-P_{e^{+}}^{L}\right) \sigma_{\mathrm{pol}}^{L}+P_{e^{-}}^{T} P_{e^{+}}^{T} \sigma_{\mathrm{pol}}^{T} .
$$


Access to the physics of the transverse cross section $\sigma_{\text {pol }}^{T}$ requires therefore that both beams be polarized.

It has been shown in [18] that transversely polarized beams project out $W_{L}^{+} W_{L}^{-}$final states, which are particularly important for studying the origin of electroweak symmetry breaking. When studying the azimuthal asymmetry, which is very pronounced at high energies reaching about $10 \%$ and peaks at larger angles, one has direct access to the $L L$ mode of $W W$ production without complicated final-state analyzes.

The azimuthal asymmetry is also a crucial observable when studying signals of extra dimension in the process $e^{+} e^{-} \rightarrow f \bar{f}[19]$. With the use of transversely polarized beams it is possible to probe spin-2 graviton exchange to about twice the sensitivity of "conventional" methods for analyzing contact interactions. In Fig. 5 the differential azimuthal asymmetry distribution is shown whose asymmetric distribution is the signal for the graviton spin-2 exchange.

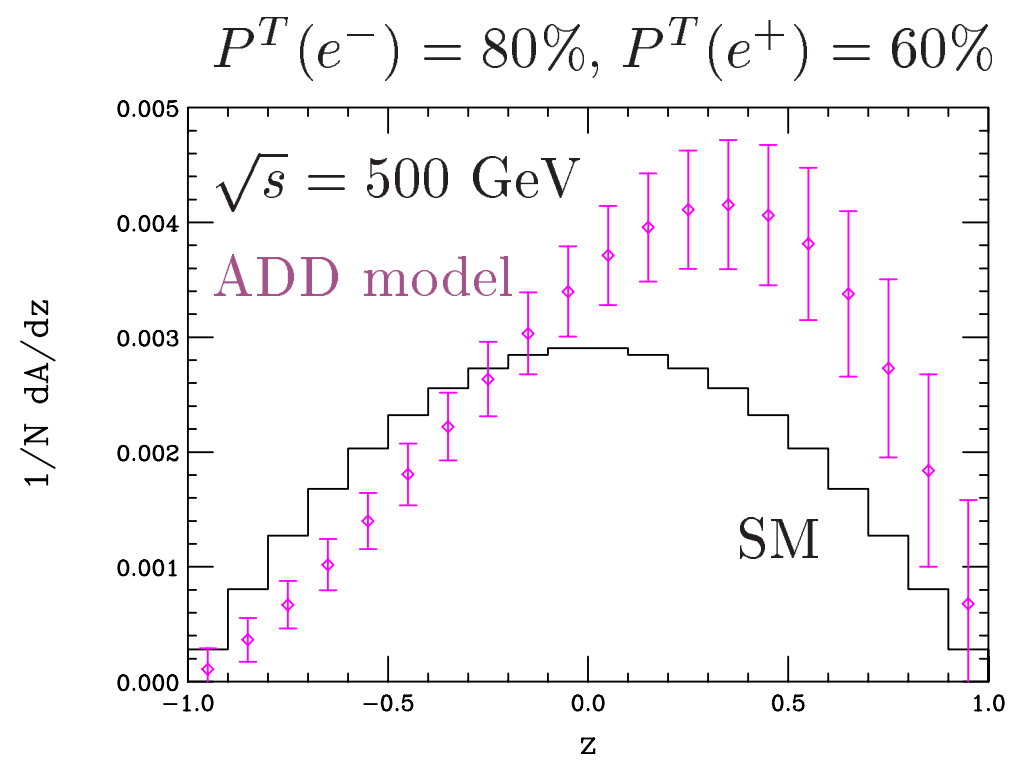

Figure 5: Search for large extra dimensions in the ADD model in $e^{+} e^{-} \rightarrow$ $f \bar{f}$ with transversely polarized beams. Shown is the differential azimuthal asymmetry distribution whose asymmetric distribution is the signal for the graviton spin-2 exchange. From [19].

\subsubsection{Precision Measurement of Beam Polarization}

A Linear Collider operating at energies around the $Z^{0}$ pole is a very powerful instrument to probe the precision structure of the standard model. Beam polarization contributes greatly to the physics potential of this option. In order to exploit this physics potential a extremely precise knowledge of the degree of polarization is needed. At the moment no method exists to directly measure the beam polarization to well below $0.1 \%$.

However if both the electron and the positron beams are polarized, the degree of polarization can be measured from the events themselves. In this so-called extended Blondel scheme 
the left-right asymmetry is directly measurable from observing counting rates in different machine configurations:

$$
A_{L R}=\sqrt{\frac{\left(\sigma^{++}+\sigma^{+-}-\sigma^{-+}-\sigma^{--}\right)\left(-\sigma^{++}+\sigma^{+-}-\sigma^{-+}+\sigma^{--}\right)}{\left(\sigma^{++}+\sigma^{+-}+\sigma^{-+}+\sigma^{--}\right)\left(-\sigma^{++}+\sigma^{+-}+\sigma^{-+}-\sigma^{--}\right)}} .
$$

With this scheme an accuracy of the electroweak mixing angle of $\delta\left(\sin ^{2} \theta_{\text {eff }}\right)=0.00001$ and $\delta\left(M_{W}\right)=6 \mathrm{MeV}[20]$ seems possible.

\subsubsection{Summary of the Physics Potential}

Although already polarized electrons by themselves offer exciting physics opportunities, the addition of polarized positrons extends the physics reach of a Linear Collider significantly by increasing the level of control one has over the mixture of processes present in the events observed. Using polarized $e^{-}$and $e^{+}$beams simultaneously improves the power to determine quantum numbers of new particles, provides higher sensitivity to non-standard model couplings and helps to reveal the structure of the underlying model. In many cases rates and purities of particular signals will be enhanced, even if only moderate (e.g. 40$60 \%$ ) positron polarization can be achieved. Additional and often unique information can be gained if not only longitudinally but also transversely polarized electrons and positrons are made available. 


\section{Principles of the Experiment}

\subsection{Overview}

This section discusses the key physical processes of E-166. As discussed above, a highenergy electron beam in combination with a helical undulator is used to produce circularly polarized photons. These photons pair-produce longitudinally polarized positrons in a thin target. The polarization of the photons is determined through measurements of the relative photon transmission through a magnetized iron absorber for positive and negative polarity of the iron magnetization. The polarization of the positrons is determined by first making polarized photons from the positrons in a reconversion target and subsequently measuring the relative transmission through a magnetized iron absorber of the regenerated photons.

For E-166, each 50-GeV electron produces on average 0.4 photons. Each photon pair produces at a rate of about $0.5 \%$ positrons/photon. The transmission efficiency of positrons to the reconversion target is about $2 \%$. Reconversion occurs at a rate of about 1 photon per positron. There is about a $0.25 \%$ transmission efficiency from the reconversion target into the detector. Thus, for $1 \times 10^{10}$ electrons per pulse, $4 \times 10^{9}$ photons per pulse are made; resulting in $2 \times 10^{7}$ positrons per pulse. Of these, $4 \times 10^{5}$ positrons per pulse are incident on the reconversion target and about $1 \times 10^{3}$ photons per pulse arrive at the detector in the positron polarimeter. In the case of the photon polarimeter, the transmission through the iron absorber is about $1 \%$, such that $4 \times 10^{7}$ photons per pulse arrive at the detector.

\subsection{Production of Circularly Polarized $\gamma$-Rays}

\subsubsection{Overview}

Polarized positrons are to be produced by conversion of circularly polarized $\gamma$-rays in a thin target. The $\gamma$-rays are the result of backscattering of an electron beam of energy $E_{e}=\gamma m c^{2}$ off the virtual photon of an undulator with period $\lambda_{u}$. To a first approximation the energy of radiation at $0^{\circ}$ is therefore

$$
E_{0} \approx \frac{2 \gamma^{2} h c}{\lambda_{u}}=24[\mathrm{MeV}] \frac{\left(E_{e} / 50[\mathrm{GeV}]\right)^{2}}{\lambda_{u}[\mathrm{~mm}]} .
$$

The highest energy radiation takes on the polarization of the undulator field, so that a helical undulator leads to circularly polarized $\gamma$-rays, the conversion of which leads to polarized positrons.

To create positrons, we need $\gamma$-rays of at least a few $\mathrm{MeV}$, so we desire the highest possible electron beam energy $E_{e}$, and the shortest possible undulator period $\lambda_{u}$. The highest practical beam energy at SLAC is $50 \mathrm{GeV}$, in which case an undulator period $\lambda_{u}$ of only 2.4 $\mathrm{mm}$ is required to obtain $10-\mathrm{MeV} \gamma$-rays.

The aperture of the undulator must be smaller than its period, and will be of order $1 \mathrm{~mm}$. Thus, the electron beam must have low emittance to pass through the undulator without scraping, and we are led to take advantage of the excellent beam quality of the SLAC Final Focus Test Beam (FFTB). 
The intensity of the $\gamma$-rays depends on the intensity of the virtual photons of the undulator, and hence on the square of its magnetic field strength. It is conventional to measure the field strength of an undulator in terms of a dimensionless parameter $K$ defined as,

$$
K=\frac{2 \pi e B_{0} \lambda_{u}}{m c^{2}}=0.09 B_{0}[\mathrm{~T}] \lambda_{u}[\mathrm{~mm}]
$$

in which $\mathrm{B}_{0}$ is the peak, transverse magnetic field in the undulator. Then the rate of undulator radiation is roughly $2 \alpha K^{2}$ per undulator period $\lambda_{u}$, where $\alpha$ is the fine-structure constant. Because of the small undulator period required for use with a $50-\mathrm{GeV}$ electron beam, the highest practical value for $K$ is about 0.2 . In this case the number of $\gamma$-rays emitted per electron in a 1-m-long undulator of $\lambda_{u}=2.4 \mathrm{~mm}$ is $2(1 / 137)(0.2)^{2}(1 / 0.0024) \approx 0.2$.

\subsubsection{Details}

Detailed descriptions of helical undulator radiation can be found in [21, 22, 23, 24, 25]. Past applications of helical undulators include the generation of $200-\mathrm{eV}$ circularly polarized photons to measure the polarization of the $650-\mathrm{MeV}$ positron beam at the VEPP-2M storage ring [26], and generation of $0.5-1 \mathrm{keV}$ photons with nearly $100 \%$ circular polarization at SLAC's SPEAR facility [27].

For small values of $K$, the number of photons $d N_{\gamma} / d L$ emitted per meter of an undulator is

$$
\frac{d N_{\gamma}}{d L}=\frac{4}{3} \frac{\pi \alpha}{\lambda_{u}} \frac{K^{2}}{1+K^{2}}=\frac{30.6}{\lambda_{u}[\mathrm{~mm}]} \frac{K^{2}}{1+K^{2}} \text { photons } / \mathrm{m} / e^{-} .
$$

The photon number spectrum is relatively flat up to the maximum energy $E_{c 10}$ of first harmonic radiation,

$$
E_{\mathrm{c} 10}=\frac{2 \gamma^{2} h c / \lambda_{u}}{1+K^{2}+2 \gamma \lambda_{C} / \lambda_{u}} \approx \frac{2 \gamma^{2} h c / \lambda_{u}}{1+K^{2}} \approx 24[\mathrm{MeV}] \frac{\left(E_{e} / 50[\mathrm{GeV}]\right)^{2}}{\lambda_{u}[\mathrm{~mm}]\left(1+K^{2}\right)}
$$

where $\lambda_{C}=h / m c=2.4 \times 10^{-12} \mathrm{~m}$ is the Compton wavelength of the electron. Small numbers of photons are emitted at higher energies, corresponding to higher multipole radiation by the electrons. The detailed photon number spectrum is illustrated in Fig. 6(a) for the proposed experimental parameters: $E_{e}=50 \mathrm{GeV}, \lambda_{u}=2.4 \mathrm{~mm}$ and $K=0.17$.

There is a fixed kinematic relation between energy and angle of emission of the photons due to $n^{\text {th }}$-order multipole radiation,

$$
E_{\gamma}(n, \theta)=\frac{n E_{\mathrm{c} 10}}{1+(\gamma \theta)^{2} /\left(1+K^{2}\right)}
$$

As seen from (9), the upper half of the energy spectrum is emitted into a cone of angle $\theta=\sqrt{1+K^{2}} / \gamma$, where $\gamma=E_{e} / m c^{2}$.

As previously mentioned, the polarization $P_{\gamma}$ of the $\gamma$-rays produced at $0^{\circ}$ is the same as that of the undulator, but falls off for larger angles (which corresponds to lower energies). This behavior is illustrated in Fig. 6(b) for the proposed experimental parameters. The polarization of higher harmonic radiation approaches unity at the corresponding higher cutoff energies, but the rates are very low there. 

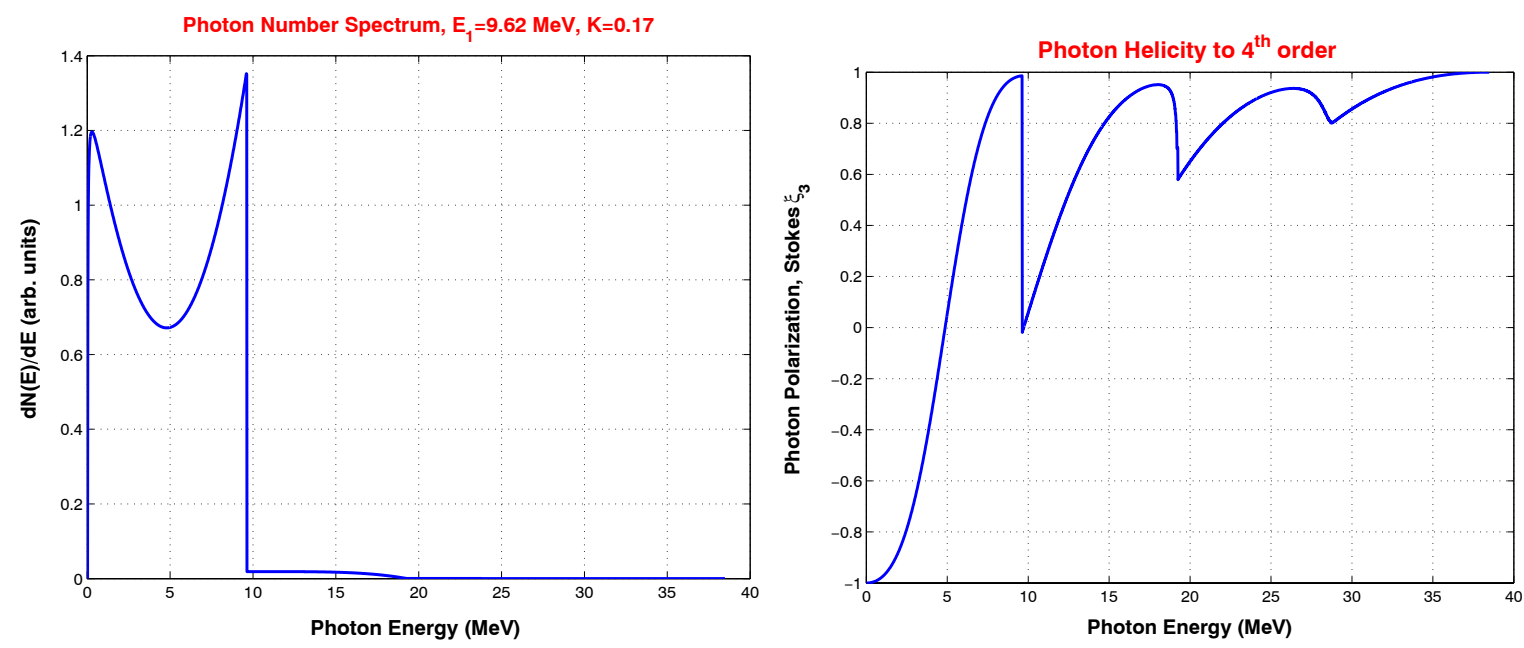

Figure 6: (a) The photon number spectrum, intensity spectrum, of undulator radiation, integrated over angle, for electron energy $E_{e}=50 \mathrm{GeV}$, undulator period $\lambda_{u}=2.4 \mathrm{~mm}$ and undulator strength parameter $K=0.17$. The peak energy $E_{c 10}$ of the first harmonic (dipole) radiation is $9.62 \mathrm{MeV}$. (b) The polarization $P_{\gamma}$ of the undulator radiation as a function of energy.

If the photons are observed in a calorimeter, an appropriate measure of their effective polarization $P_{\gamma}$ is the energy-weighted average polarization, obtained by multiplying the number of photons of energy $E$ by $E$ times the polarization at that energy. For the undulator photons considered here, the energy-weighted polarization is $49 \%$.

The radiated power $d^{2} U_{u} / d L d t$ per meter of undulator by an electron beam is

$$
\frac{d^{2} U_{u}}{d L d t}=2.32 \times 10^{-4} \frac{E_{e}^{2}[\mathrm{GeV}] K^{2}}{\lambda_{u}^{2}[\mathrm{~mm}]} n_{e}\left[\times 10^{10}\right] f_{\mathrm{rep}}[\mathrm{Hz}] \quad \mathrm{W} / \mathrm{m},
$$

in which $n_{e}$ is the number of electrons per pulse and $f_{\text {rep }}$ is the pulse repetition rate.

Figure 7 illustrates the $\gamma$-ray intensity spectrum (integrated over $0<\theta<\pi / 2$ ) and angular distribution of undulator radiation for the proposed experimental parameters. A small contribution of quadrupole (2nd harmonic) and higher-order radiation can be seen for energies of 10-20 MeV, which peaks at a nonzero angle of emission.

\subsection{Production of Polarized Positrons}

\subsubsection{Overview}

When a circularly polarized photon creates an electron-positron pair in a thin target, the polarization state of the photon is transferred to the outgoing leptons according to the cross sections derived by Olsen and Maximon in 1959 [28]. Positrons with an energy close to the energy of the incoming photons are 100\% longitudinally polarized, while positrons with a lower energy have a lower longitudinal polarization (see Fig. 8). At energies below $25 \%$ of the photon energy the sign of the positron polarization is opposite to that of the photon. 

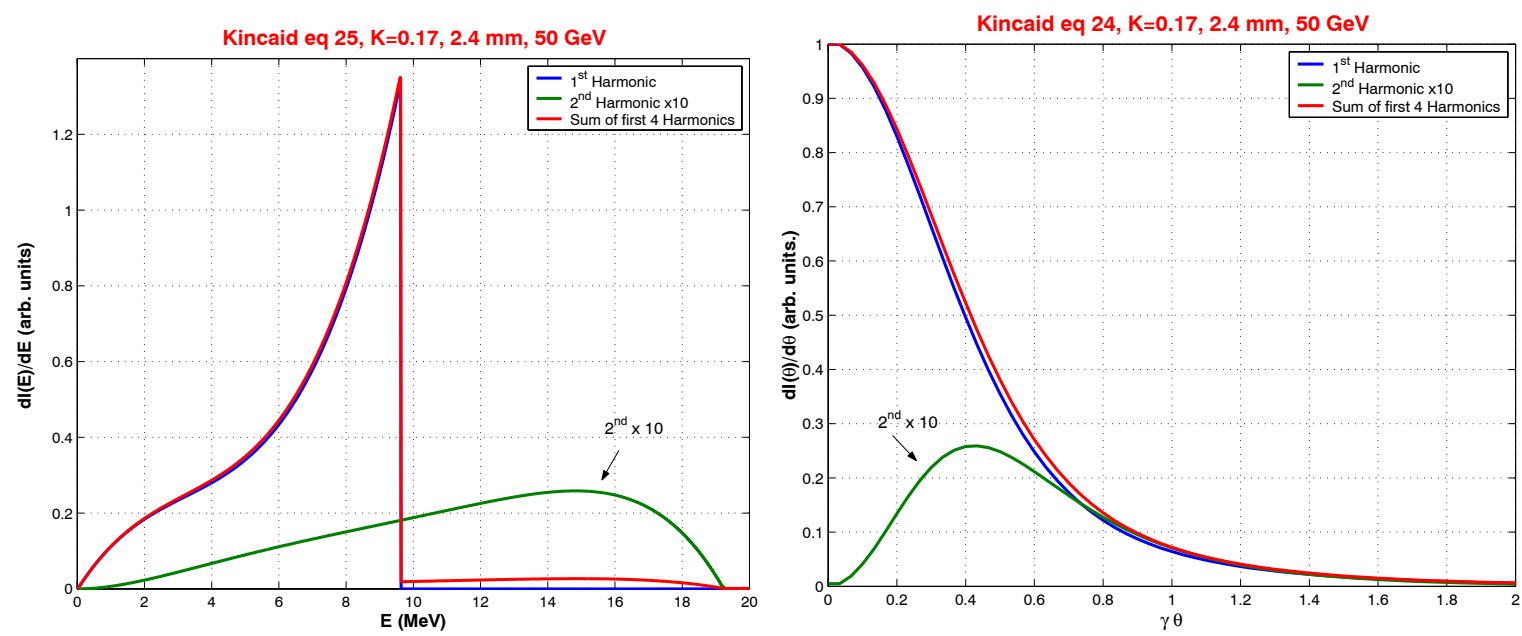

Figure 7: (a) The intensity spectrum of undulator radiation, integrated over angle, for a 50-GeV electron beam incident on an undulator with period $\lambda_{u}=$ $2.4 \mathrm{~mm}$ and strength parameter $K=0.17$. (b) Intensity, integrated over energy, vs. emission angle $\gamma \theta$.

The probability for the production of positrons is roughly independent of the fractional energy $E_{e^{+}} / E_{\gamma}$ in the pair-production process, so that positrons with all energies up to the photon energy are produced (with initial polarization as shown in Fig. 8). However, even in a thin target, low-energy positrons are stopped due to the ionization loss (which rises sharply for energies below $1 \mathrm{MeV}$ ), while high-energy positrons loose a fraction of their energy due to bremsstrahlung. The energy loss by bremsstrahlung is accompanied by a slight loss of polarization; however, the energy loss is stronger than the polarization loss. As a result, the low-energy portion of the positron spectrum is repopulated with positrons from the higher energy portion, and the polarization of positrons of a given energy is higher in targets of up to $\approx 0.5$ radiation length than in an infinitely thin target [4], as shown in Fig. 9 .

For targets thicker than about 0.5 radiation length the polarization decreases again. Hence, positrons are unpolarized in a conventional thick-target positron source even if the incoming electrons are polarized. (At very low yield polarized positrons may also be produced from polarized electrons using thin targets [29].)

\subsubsection{Details}

The basic processes of polarized electromagnetic cascades are well known, but understanding of the interplay of all processes in a shower requires simulation with a Monte-Carlo code. To this end, the programs EGS4 [29, 30] and GEANT3 [31] have been modified to include effects of polarization. ${ }^{2}$

The polarized versions of these codes include the effects for pair production, bremsstrahlung and Compton scattering (with the exception of scattering asymmetries which are not consid-

\footnotetext{
${ }^{2}$ Improvements of the polarized Monte-Carlo codes are ongoing, in parallel to the preparation of the experiment, in cooperation with colleagues from Byelorussia.
} 


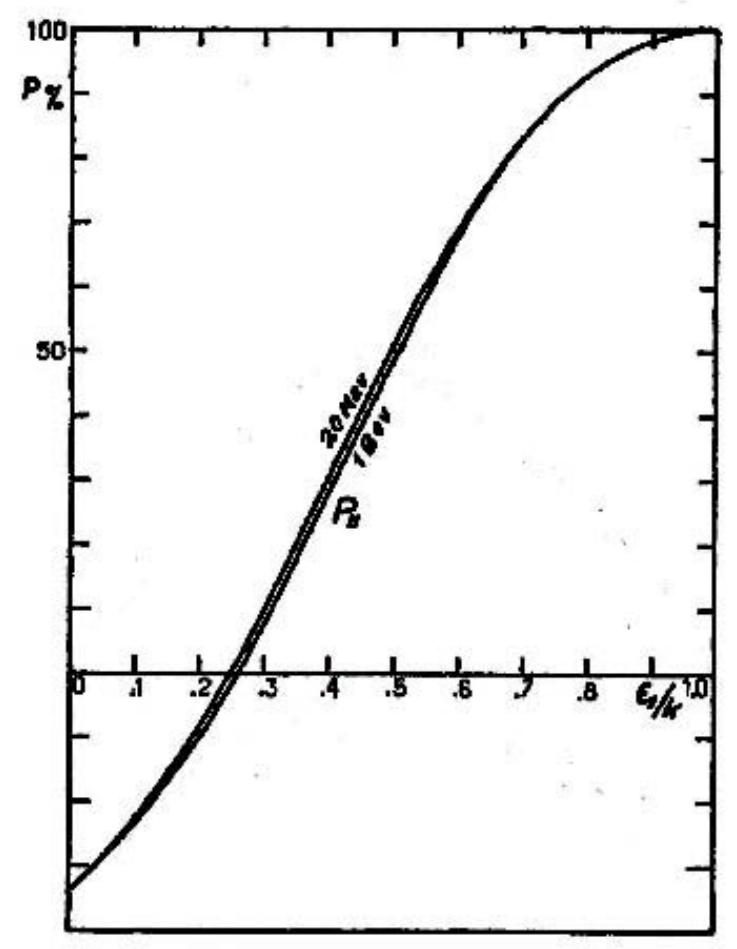

Figure 8: Longitudinal polarization of positrons (or electrons) produced by conversion of monochromatic circularly polarized photons in an infinitely thin target, as a function of the ratio of positron to photon energies. From [28].

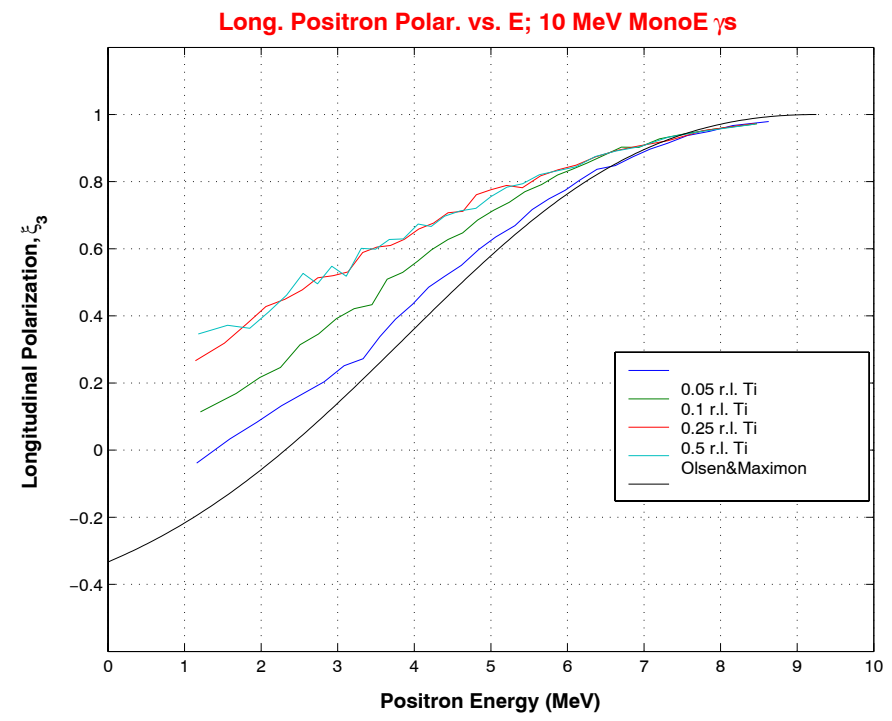

Figure 9: Longitudinal polarization of positrons produced by conversion of 10$\mathrm{MeV}$ circularly polarized photons in targets of various thickness in radiation lengths, as a function of the positron energy. 
ered) [4]. The effects of other processes on the polarization, e.g., multiple Coulomb scattering, are not taken into account yet. A semi-classical approach is followed, by assigning an average polarization to individual particles. The polarized cross sections of Olsen and Maximon [28] for pair production and bremsstrahlung and of Lipps and Tollhoek [32] for Compton scattering are utilized. Various simplifications have been made in the simulations; for example, a $1 / \gamma$ angular distribution of the outgoing particles is assumed for the bremsstrahlung and pair production cross sections by Olsen and Maximon, while EGS4 offers more accurate angular sampling at lower energies.
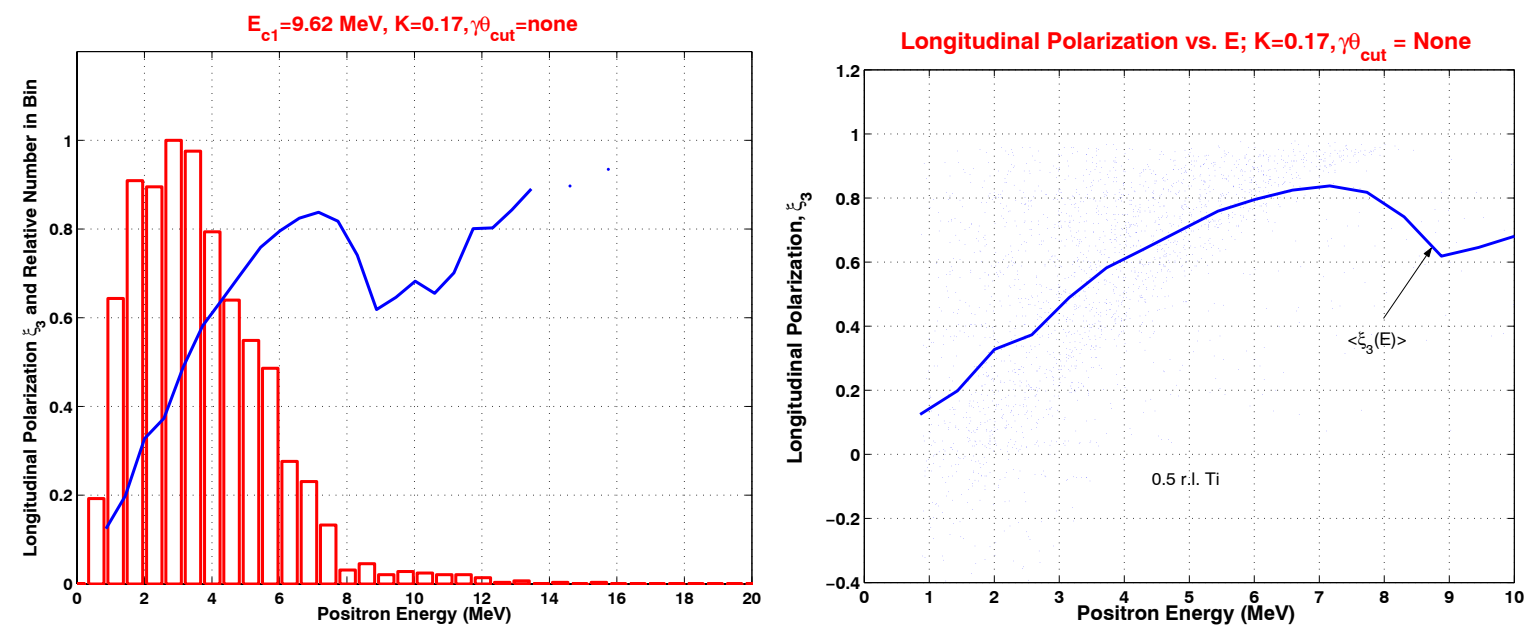

Figure 10: (a) Longitudinal polarization (solid curve) and energy spectrum (histogram) of positrons emitted from a 0.5-rad.-len.-thick Ti target that has been irradiated with the photon energy and polarization spectra of Figs. 6 and 7. (b) Positron longitudinal polarization as a function of energy. The solid lines in (a) and (b) are polarization averaged over a $0.5-\mathrm{MeV}$ energy slice. The dip in the polarization at $9 \mathrm{MeV}$ is due to the corresponding dip in photon polarization at about $10 \mathrm{MeV}$ as seen in Fig. 6(b).

Figure 10 shows the energy and polarization spectra of positrons produced in $0.5 \mathrm{rad}$. len. of Ti by photons from a helical undulator whose spectra are shown in Figs. 6a and 6b. The solid curve in each plot is the average polarization within a $0.5-\mathrm{MeV}$ energy slice. As expected, the higher-energy positrons have generally higher polarization. The dip in the polarization, at positron energies of about $9 \mathrm{MeV}$, is due to the corresponding dip in photon polarization at $10 \mathrm{MeV}$ as seen in Fig. 6(b). The composite polarization of the total sample of positrons in Figures 10 is about $53 \%$.

Figure 11 shows energy spectra of positrons for different lengths of a Ti convertor by undulator photons whose spectra are shown in Figs. 6 and 7. As the target thickness increases, the positron yield initially improves but decreases for a thickness of more than $0.25 \mathrm{rad}$. len. The energy spectra do not vary significantly with target thickness. For the conditions of Fig. 11, the yield is in the range of $0.3-0.5 \%$ positrons/photon for Ti thicknesses of $0.05-0.5$ rad. len.

The conversion efficiency from low-energy $\gamma$-rays to positrons in a thin target will be 

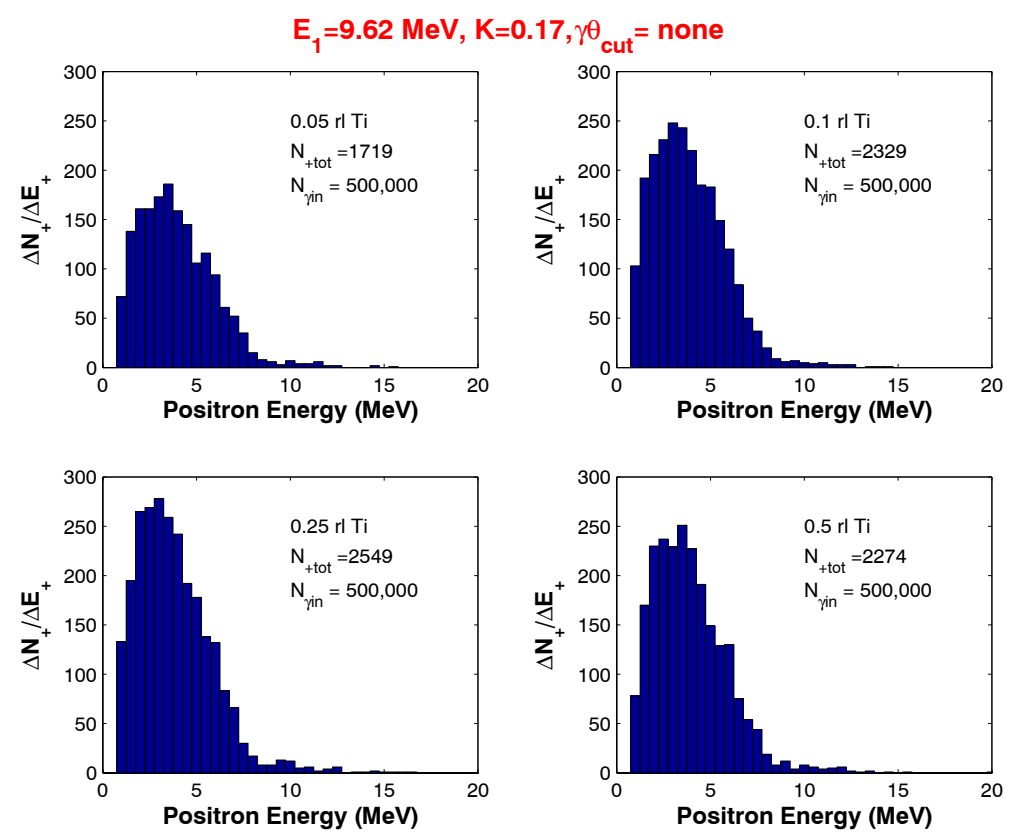

Figure 11: Positron energy spectrum for Ti targets of thickness 0.05, 0.1, 0.25, and $0.5 \mathrm{rad}$. len. The target thickness, total number of emitted positrons, and total number of incident photons are indicated in each frame. The incident photon spectrum is shown in Fig. 6(a) and 7(a).

about 0.005 , so the number of positrons produced per beam electron will be about 0.001 .

\subsection{Polarimetry of MeV $\gamma$-Rays}

Measurements of the circular polarization of energetic photons are most commonly based on the spin dependence of Compton scattering off atomic electrons [33, 34]. One can either observe the scattered electrons and/or photons emerging from a thin, magnetized iron foil [35], or measure the transmission of unscattered photons through a thick, magnetized iron absorber [36, 37, 38].

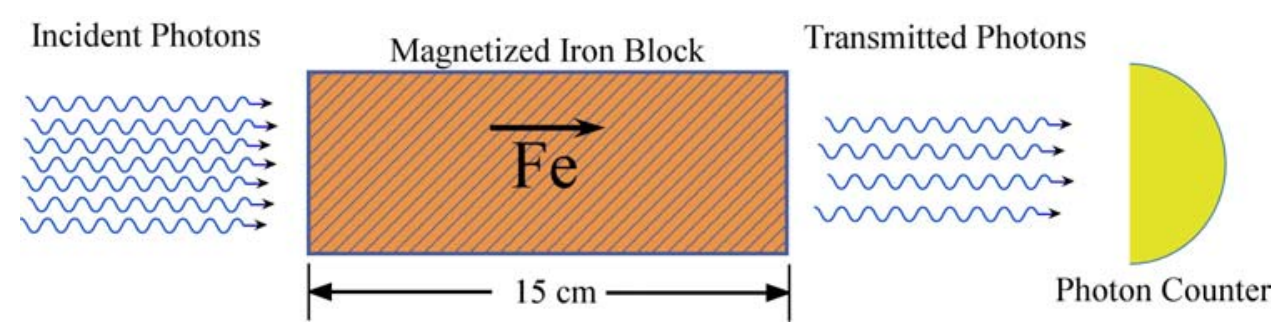

Figure 12: The concept of transmission polarimetry, in which the survival rate is measured for photons that pass through a magnetized iron absorber. 
Because of its simplicity, we will use the latter technique, transmission polarimetry, whose concept is sketched in Fig. 12. In the first approximation, a single Compton scatter of a photon removes it from the transmitted signal. The Compton scattering cross section can be written

$$
\sigma=\sigma_{0}+P_{\gamma} P_{e} \sigma_{P}
$$

where $\sigma_{0}$ is the unpolarized cross section,

$$
\sigma_{0}=\frac{\pi r_{0}^{2}}{k_{0}}\left[\left(1-\frac{2}{k_{0}}-\frac{2}{k_{0}^{2}}\right) \ln \left(1+2 k_{0}\right)+\frac{1}{2}+\frac{4}{k_{0}}-\frac{1}{2\left(1+2 k_{0}\right)^{2}}\right]
$$

$P_{\gamma}$ is the net polarization of the photons, $P_{e}$ is the net polarization of the atomic electrons (naively $\pm 2 / 26$ for $\mathrm{Fe}$, but more accurately determined to be $\pm 7.92 \%$ for iron at saturation), and $\sigma_{P}$ is the polarized cross section given by

$$
\sigma_{P}=\frac{2 \pi r_{0}^{2}}{k_{0}}\left[\frac{1+4 k_{0}+5 k_{0}^{2}}{\left(1+2 k_{0}\right)^{2}}-\frac{1+k_{0}}{2 k_{0}} \ln \left(1+2 k_{0}\right)\right] \text {, }
$$

where $r_{0}=e^{2} / m c^{2}$ is the classical electron radius and $k_{0}=E_{\gamma} / m c^{2}$. The cross sections $\sigma_{0}$ and $\sigma_{P}$ for iron are shown in Fig 13, and the attenuation of photons in iron based on cross section $\sigma_{0}$ is illustrated in Fig. 14.
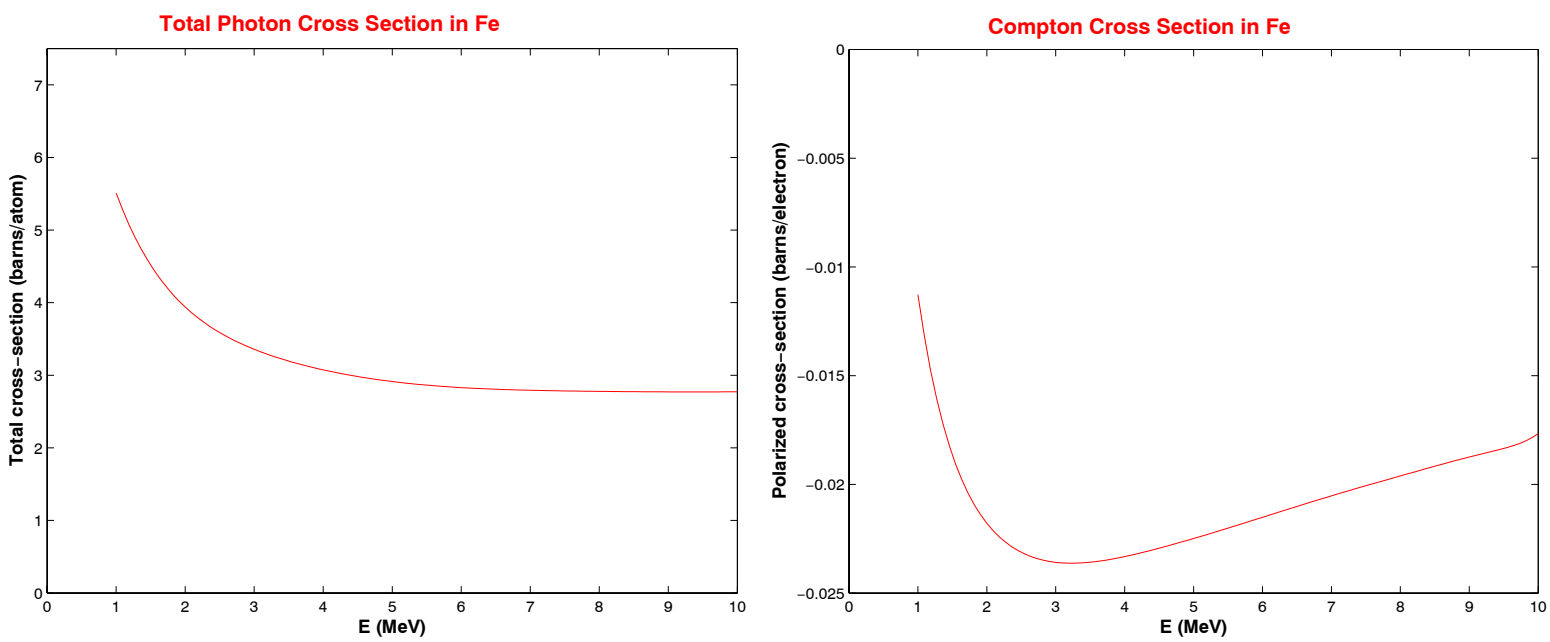

Figure 13: (a) The unpolarized photon cross section $\sigma_{0}$ per iron atom, and (b) the polarized Compton cross section $\sigma_{P}$ per electron in iron. From [39].

The transmission probability $T^{ \pm}(L)$ for photons of helicity $-P_{\gamma}$ through a piece of magnetized iron whose length is $L$ can be written as

$$
T^{ \pm}(L)=e^{-n L \sigma}=e^{-n L \sigma_{0}} e^{ \pm n L P_{e} P_{\gamma} \sigma_{P}},
$$

where $n$ is the number density of atoms in iron and the $+(-)$ in $T^{ \pm}$applies if the electron spin in the iron is parallel (antiparallel) to the direction of the incident photons. The asymmetry 

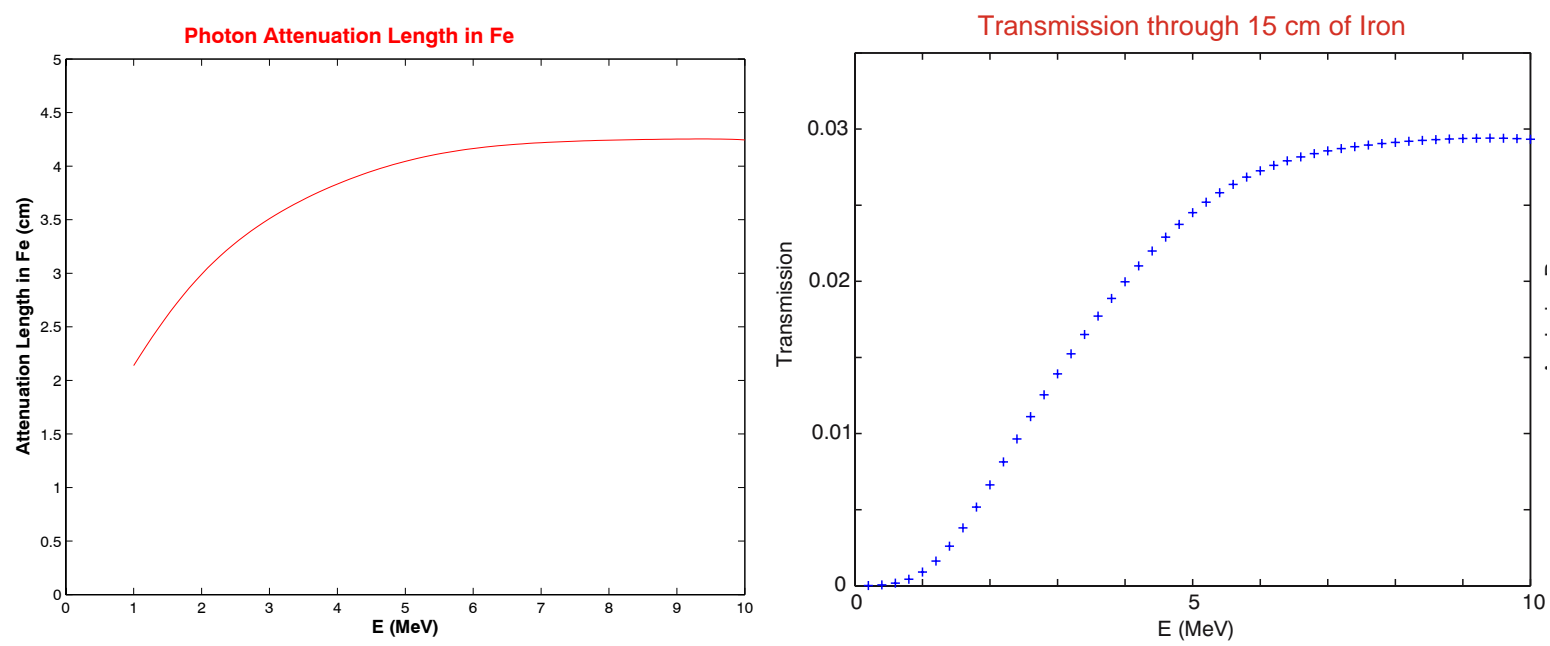

Figure 14: (a) The attenuation length for $\mathrm{MeV}$ photon in iron, based on the unpolarized Compton cross section (8). (b) The transmission of unpolarized photons through $15 \mathrm{~cm}$ of iron.

$\delta$ in transmission of photons through the iron absorber when the sign of $P_{e}$ is reversed, corresponding to a reversal of the magnetization of the iron, is

$$
\delta(L)=\frac{T^{+}(L)-T^{-}(L)}{T^{+}(L)+T^{-}(L)}=\tanh \left(n L P_{e} P_{\gamma} \sigma_{P}\right) \approx n L P_{e} P_{\gamma} \sigma_{P}
$$

This asymmetry is shown in Fig. 15(a) for various lengths of iron. The peak asymmetry is in the range of 1-6\% for photon energies in the range of several $\mathrm{MeV}$ and for lengths of iron of $3-15 \mathrm{~cm}$.

For small asymmetries such that the final form of eq. (15) holds, we can define an "analyzing power" $A_{\gamma}$ for transmission polarimetry according to

$$
A_{\gamma}(L) \equiv \frac{\delta(L)}{P_{e} P_{\gamma}} \approx n L \sigma_{P}
$$

Then, a measurement of the asymmetry $\delta$, plus knowledge of the electron polarization $P_{e}$ in the magnetized iron, determines the photon polarization to be

$$
P_{\gamma}=\frac{\delta}{P_{e} A_{\gamma}} .
$$

The relative error on a measurement of the polarization varies inversely as the product of the analyzing power $A_{\gamma}$ and the square root of the transmission factor $T$. We can therefore define a figure of merit for transmission polarimetry as $A_{\gamma}^{2} T$, where larger values are better. This figure of merit is shown in Fig. 15(b) for 7.5-MeV photons. We see that at this energy an 8-cm-long magnetized iron absorber is optimal. 

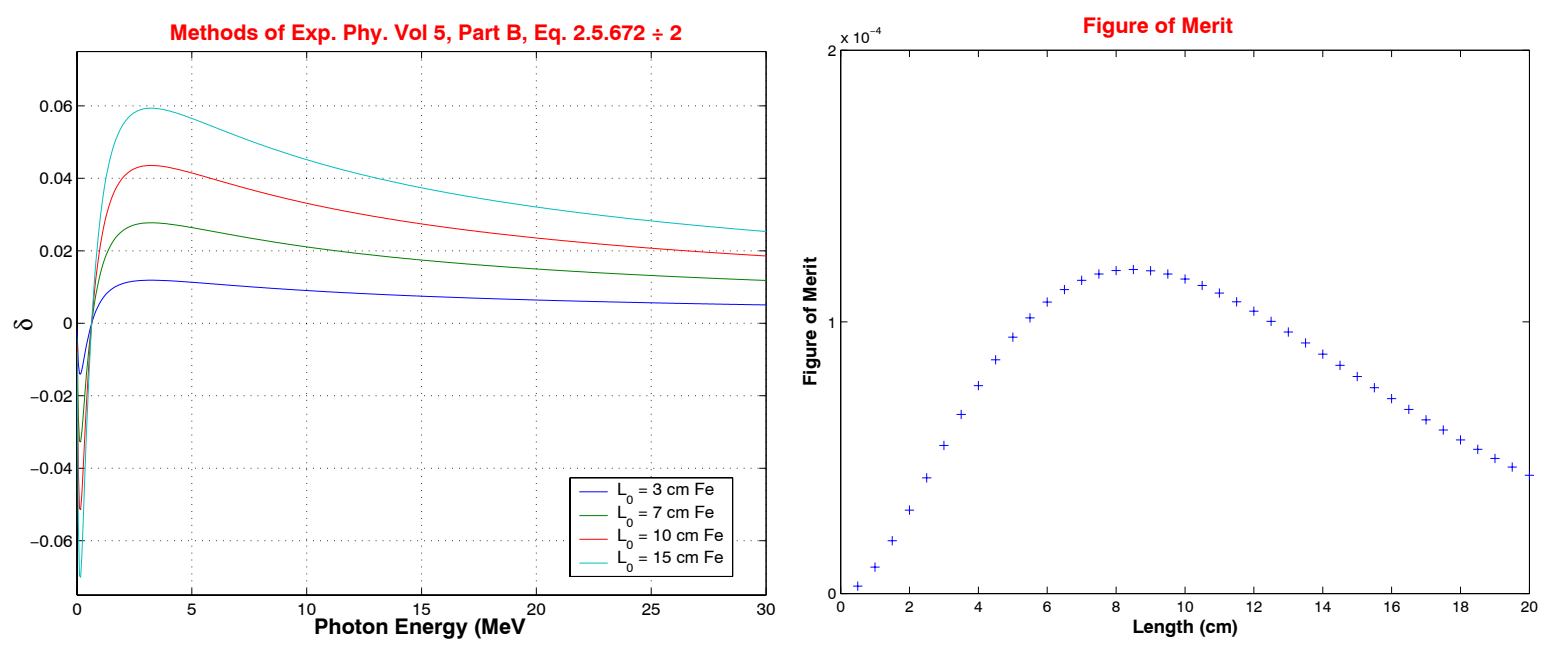

Figure 15: (a) The asymmetry $\delta$ defined in eq. (15) for transmission polarimetry of $\mathrm{MeV}$ photons in various lengths of iron. (b) The figure of merit $A_{\gamma}^{2} T$ for 7.5 MeV photons as a function of the length of a transmission iron polarimeter

\subsection{Polarimetry of MeV Positrons}

The polarization of the positrons can be analyzed by measurement of asymmetries in annihilation from $[28,40,41,42,47]$, or Bhabha scattering off [43, 44, 45, 46, 47], polarized electrons in a thin iron foil, and in Compton scattering off a circularly polarized laser [26]. Good precision can be obtained with a thin iron foil when a coincidence experiment is performed [47]. However, the simplest technique applicable to a high rate environment (where coincidences cannot be identified) is the method of transmission polarimetry in which the positrons are converted back into photons (either by annihilation [40, 41] or by bremsstrahlung $[28,48,49])$ and the latter are sent through a thick iron absorber $[50,51,52,53,54,55]$. A measurement of the transmission asymmetry for magnetic fields (in the iron) parallel and antiparallel to the positron beam direction then allows one to infer the polarization of the positrons.

The positrons can, in principle, become depolarized by atomic interactions prior to the emission of the photons that pass through the transmission polarimeter. However, this effect is at the level of a few percent for relativistic electrons [56, 57, 58], and is simulated in the EGS4 code that includes polarization.

The transfer of polarization from positrons to photons ("reconversion") in a thin foil is illustrated in Fig. 16. The average polarization of the photons from a $10-\mathrm{MeV}$ positron is only $21 \%$ of that of the positron.

The photons that have been created by the positrons can then be analyzed in a transmission polarimeter as discussed in sec. 2.5. An asymmetry $\delta$ in the number of transmitted photons is measured by reversing the polarization $P_{e^{-}}$of the electrons in the iron absorber. 


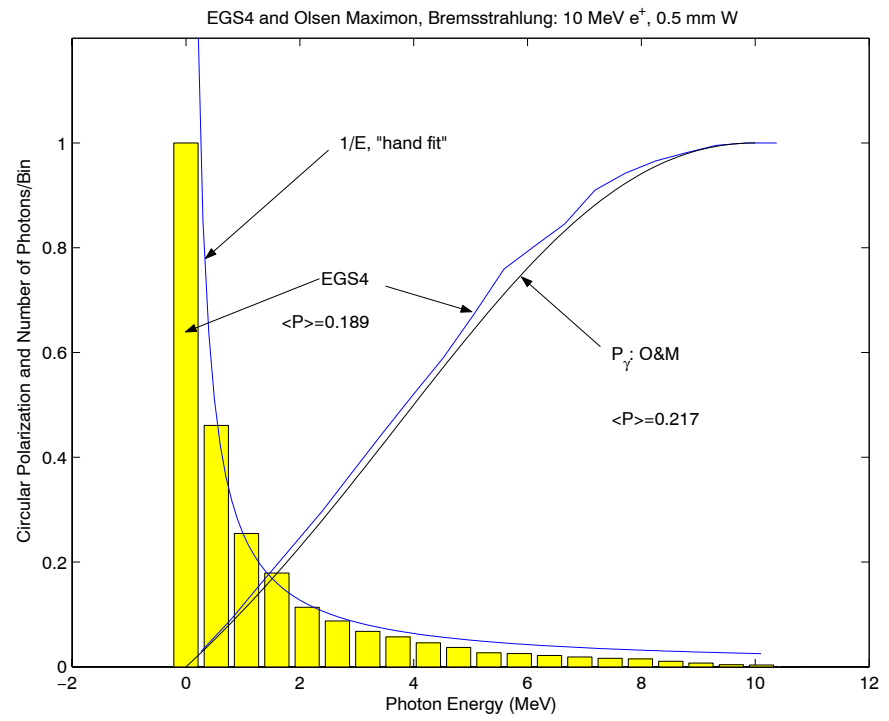

Figure 16: Solid curve: the polarization of photons generated by a 10$\mathrm{MeV}$ positron incident on $0.5 \mathrm{~mm}$ of tungsten, as a function of photon energy. Histogram: the energy spectrum the photons, which are mainly due to bremsstrahlung.

The polarization $P_{e^{+}}$of the parent positrons can then be inferred according to

$$
P_{e^{+}}=\frac{\delta}{P_{e^{-}} A_{e^{+}}},
$$

in terms of an analyzing power $A_{e^{+}}$that can be calculated in a simulation that combines the processes of polarization transfer from positron to photon and transmission of the photons through the iron absorber. Because the reconverted photons have a nearly isotropic angular distribution (due to the large multiple scattering of the parent positrons in the reconversion target), the computation of the analyzing power $A_{e^{+}}$is more complicated than for $A_{\gamma}$ in the case of transmission polarimetry of a collimated photon beam.

Figure 17 shows the analyzing power $A_{e^{+}}$for the example of a $7.5-\mathrm{cm}-$ long iron absorber and the polarimeter geometry shown in Fig. 19 and 26. 


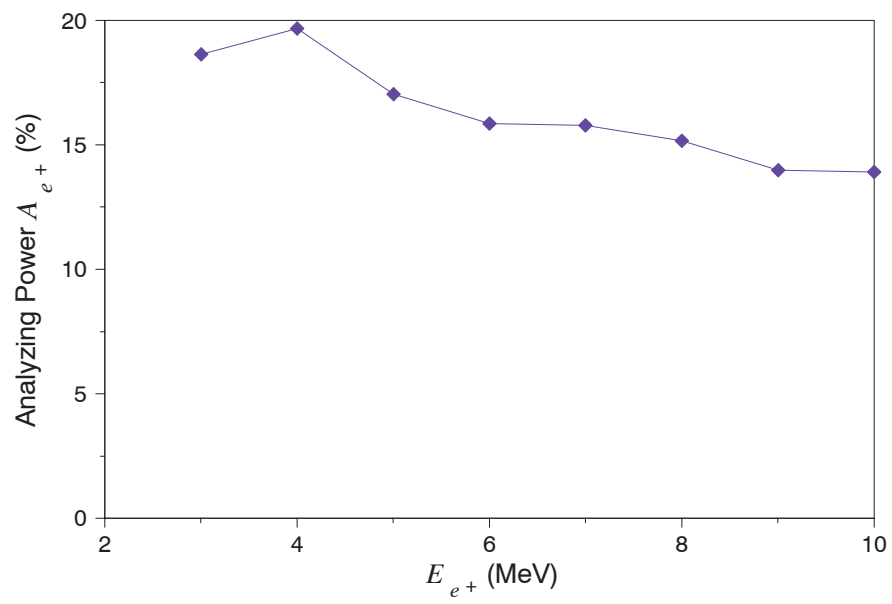

Figure 17: The analyzing power $A_{e^{+}}$for positron polarimetry as a function of positron energy, when the positrons are reconverted into photons that pass through a 7.5 -cm-long magnetized iron absorber and the polarimeter geometry shown in Fig. 19 and 26. 


\section{The Apparatus}

\subsection{Overview}

The goal of the experiment is

- To measure the yield and polarization of the photons produced by passing an electron beam through a helical undulator.

- To measure the yield and polarization of the positrons produced by conversion of undulator photons in a thin target.

- To compare the results to simulations.

A schematic layout of the experiment is shown in Fig. 18 with emphasis on the particle beams, while Fig. 19 shows the layout of the detectors to measure the flux and polarization of the photons and positrons.

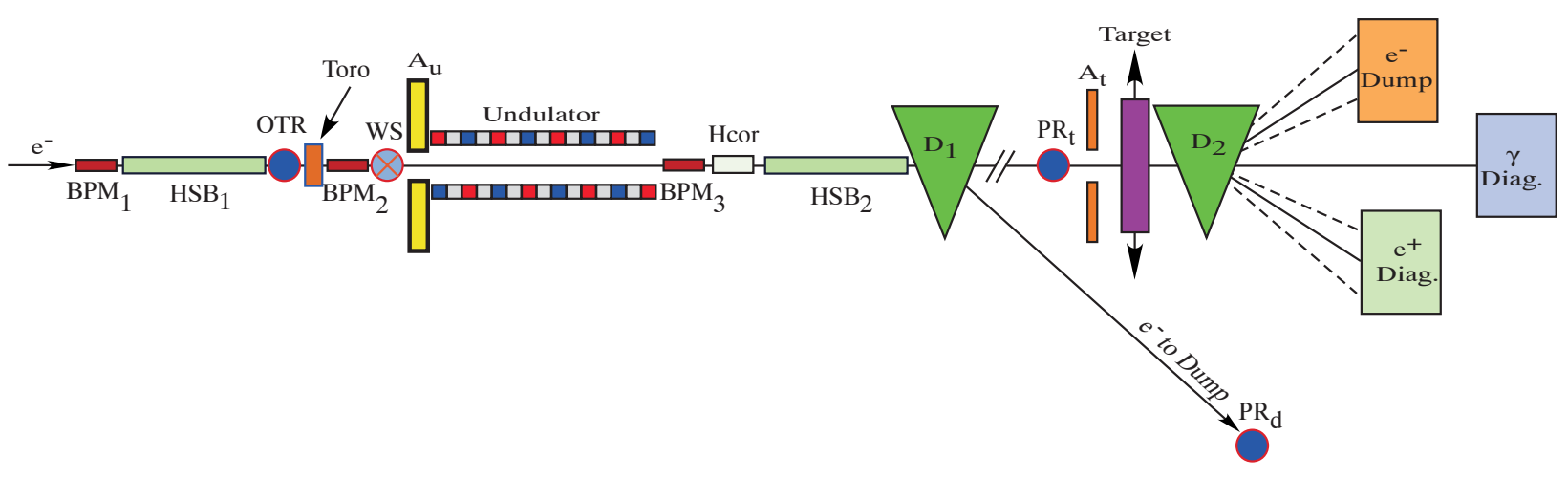

Figure 18: Conceptual layout (not to scale) of the experiment to demonstrate the production of polarized positrons in the SLAC FFTB. 50-GeV electrons enter from the left and are dumped using magnet $\mathrm{D}_{1}$ after traversing the undulator. The positron conversion target as well as the positron and photon diagnostics are located $35 \mathrm{~m}$ downstream of the undulator. $\mathrm{BPM}_{i}=$ beam-position monitor; $\mathrm{HSB}_{i}=$ "hard" soft bend; OTR = optical-transition-radiation beamprofile monitor; Toro = beam-current toroid; WS = wire scanner; $\mathrm{A}_{i}=$ aperture limiting collimators; Hcor $=$ horizontal steering magnet; $\mathrm{D}_{1}=$ FFTB primary beam dump bend-magnet string; $\mathrm{PR}_{d}=$ dumpline beam-profile monitor; $\mathrm{PR}_{t}=e^{+}$target beam-profile monitor; $\mathrm{D}_{2}=$ analyzing magnet.

The experiment uses a low-emittance, 50-GeV electron beam (sec. 3.2.2) in the SLAC Final Focus Test Beam (FFTB) plus a 1-meter-long, short-period $\left(\lambda_{u}=2.4\right.$-mm, $\left.K=0.17\right)$, pulsed helical undulator (sec. 3.3), to produce circularly polarized photons of energies up to $10 \mathrm{MeV}$. These polarized photons are then converted to polarized positrons through pair production in a Ti target which has a nominal thickness of $0.5 \mathrm{rad}$. len. The polarizations 


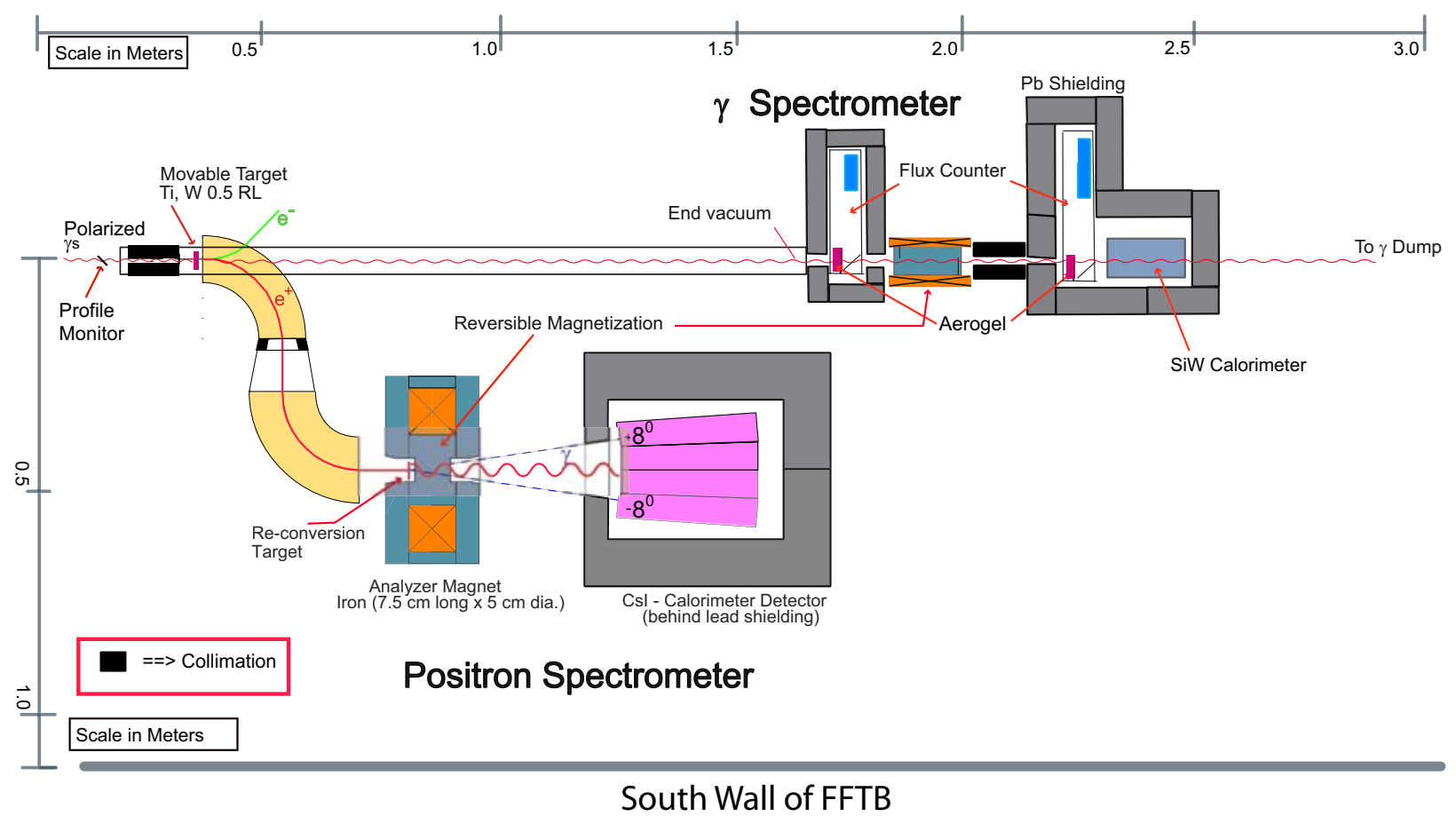

Figure 19: Conceptual layout of the $\mathrm{E}-166$ positron generation and photon and positron diagnostic systems.

of the photons and positrons are measured by the Compton transmission method using a magnetized iron absorber [37].

This experiment is a demonstration of undulator-based production of polarized positrons for Linear Colliders at a scale of $1 \%$ in length and intensity:

- Photons are produced in the same energy range and polarization as in a Linear Collider;

- The same target thickness and material are used as in the Linear Collider;

- The polarization of the produced positrons is expected to be in the same range as in a Linear Collider.

- The simulation tools being used to model the experiment are the same as those being used to design the polarized positron system for a Linear Collider: EGS4 [30] and GEANT3, both modified to include spin effects for polarized $e^{+}$production, and BEAMPATH [59] for collection and transport. 


\subsection{The Beamline}

\subsubsection{Layout}

Figure 18 shows the layout of the proposed experiment in the SLAC FFTB. 50-GeV, low-emittance electrons are sent through a helical undulator to produce circularly polarized photons. After the undulator, the $50-\mathrm{GeV}$ electrons are bent vertically downward and sent to the FFTB dump. The photons drift in the zero-degree line for a distance of about $35 \mathrm{~m}$ where they are either analyzed or converted to positrons in a thin target.

\subsubsection{Beam Parameters}

Table 5 lists the requested E-166 beam parameters. Radiation shielding considerations limit the maximum beam power in the FFTB enclosure to less than $2.5 \mathrm{~kW}$. For $50-\mathrm{GeV}, 30$ $\mathrm{Hz}$ operation this corresponds to a beam current $\leq 1 \times 10^{10} e^{-} /$pulse. The emittances of the electron beam for fully coupled damping ring operation, and low beam charge, are expected to be less than $\gamma \epsilon_{x}=\gamma \epsilon_{y}=3.0 \times 10^{-5} \mathrm{~m}$-rad or less. For $\beta$ of $5.2 \mathrm{~m}$, the corresponding beam size is about $40 \mu \mathrm{m}$ (rms); the angular divergence of the electron beam at the undulator is smaller than the characteristic angular spread $1 / \gamma$ of the undulator radiation (see Table 5 ).

Table 5: E-166 beam parameters.

\begin{tabular}{|c|c|c|c|c|c|c|}
\hline \hline$E_{e}$ & $f_{\text {rep }}$ & $N_{e}$ & $\gamma \epsilon_{x}=\gamma \epsilon_{y}$ & $\beta_{x}, \beta_{y}$ & $\sigma_{x}, \sigma_{y}$ & $\sigma_{E} / E$ \\
\hline \hline $\mathrm{GeV}$ & $\mathrm{Hz}$ & $e^{-}$ & $\mathrm{m}-\mathrm{rad}$ & $\mathrm{m}$ & $\mu \mathrm{m}$ & $\%$ \\
\hline 50 & 30 & $1 \times 10^{10}$ & $3 \times 10^{-5}$ & $5.2,5.2$ & 40 & 0.3 \\
\hline \hline
\end{tabular}

The requested beam energy of $50 \mathrm{GeV}$ is necessary to produce the highest possible photon energy. Recent experience with E158 shows that a nominal energy of $50 \mathrm{GeV}$ at the end of the linac is possible at the requested bunch charge of $1 \times 10^{10}$ electrons per bunch with 16 spare klystrons (maximum linac energy of $54 \mathrm{GeV}$ at $1 \times 10^{10}$ electrons per bunch and $\left.\sigma_{z}=500 \mu \mathrm{m}\right)$. This number of spare klystrons is sufficient for continuous E-166 operation with negligible interruption from beam energy issues.

The requested $30-\mathrm{Hz}$ rate and charge of $1 \times 10^{10}$ electrons per bunch matches the FFTB radiation shielding design limit at $50 \mathrm{GeV}$. Neither the rate nor the charge per bunch goals are not expected to be difficult to achieve. Additional electrical power costs for $30 \mathrm{~Hz} v s$. a lower repetition rate are offset by the water-heater loads required to set the accelerator structure operating temperature. A 1-Hz keep-alive rate during PEP II fills is important for maintaining the beam trajectory and quality to ensure rapid resumption of E-166 upon completion of ring fills. 
Table 6: E-166 photon beam parameters for an undulator strength parameter of $K=0.17$ and distance from undulator to positron production target of $D=35 \mathrm{~m} . \sigma_{\text {eff }}^{\prime}=\left(1 / \gamma^{2}+\sigma_{x}^{2} / D^{2}+\sigma_{x^{\prime}}^{2}\right)^{1 / 2}$ and $\sigma_{t}=D / \gamma_{\mathrm{eff}}$.

\begin{tabular}{|r|c|c|c|c|c|c|c|c|}
\hline \hline$E_{e}$ & $E_{c 10}$ & $\gamma \epsilon_{x}=\gamma \epsilon_{y}$ & $\beta_{x}, \beta_{y}$ & $\sigma_{x}, \sigma_{y}$ & $\sigma_{x^{\prime}}, \sigma_{y^{\prime}}$ & $1 / \gamma$ & $\sigma_{\text {eff }}^{\prime}$ & $\sigma_{t}$ \\
\hline $\mathrm{GeV}$ & $\mathrm{MeV}$ & $\mathrm{m}-\mathrm{rad}$ & $\mathrm{m}$ & $\mu \mathrm{m}$ & $\mu \mathrm{rad}$ & $\mu \mathrm{rad}$ & $\mu \mathrm{rad}$ & $\mu \mathrm{m}$ \\
\hline \hline 50 & 9.62 & $2 \times 10^{-5}$ & $7.8,7.8$ & 40 & 5.1 & 10.2 & 11.5 & 402 \\
\hline 50 & 9.62 & $4 \times 10^{-5}$ & $3.9,3.9$ & 40 & 10.2 & 10.2 & 14.5 & 507 \\
\hline 47.5 & 8.68 & $2 \times 10^{-5}$ & $7.4,7.4$ & 40 & 5.4 & 10.8 & 12.1 & 423 \\
\hline 47.5 & 8.68 & $4 \times 10^{-5}$ & $3.7,3.7$ & 40 & 10.8 & 10.8 & 15.3 & 534 \\
\hline \hline
\end{tabular}

A limiting constraint for E-166 is the $0.885 \mathrm{~mm}$ I.D. aperture of the undulator. To prevent background generation due to beam interception, a beam size of $40 \mu \mathrm{m}$ rms has been adopted. This gives an undulator radius-to-beam size ratio of $11 \sigma$. To achieve this beam size at $50 \mathrm{GeV}$, the $\beta$ functions at IP1 must be set to the range of $7.8 \mathrm{~m}$ to $3.9 \mathrm{~m}$ for $\gamma \epsilon=2-4 \times 10^{-5} \mathrm{~m}$-rad. The computer code DIMAD has been run to find magnet values for $\beta$ function at IP1 of $10 \mathrm{~m}$ and $2.5 \mathrm{~m}$. The required magnet strengths are well within the magnet fabrication specifications and power supply operating ranges. An emittance at the lower end of the expected tuning range, $\gamma \epsilon=2 \times 10^{-5} \mathrm{~m}$-rad, is preferred for ease of attaining the $40-\mu \mathrm{m}$ rms beam size through the undulator.

A nominal value of $\gamma \epsilon=3 \times 10^{-5} \mathrm{~m}$-rad is listed in Table 5 . For the purpose of making comparisons, Table 6 summarizes the effects of the range of electron beam emittance on the E-166 photon beam energy $E_{c 10}$ (first harmonic cutoff), angular divergence $\sigma_{e f f}^{\prime}$, and spot size at the converter target $\sigma_{t}$ at beam energies of $50 \mathrm{GeV}$ and $47.5 \mathrm{GeV}$. In Table 6 , the $\beta$ function at IP1 is adjusted to keep a $40 \mu \mathrm{m}$ electron beam size through the undulator.

An rms energy spread of $\sigma_{E} / E \leq 0.3 \%$ is a factor of 1.5-2 times larger than expected for the nominal bunch current with an rms bunch length of $\sigma_{z}=500 \mu \mathrm{m}$. This requirement on the energy spread is to limit the possibility of background generation in the FFTB due to beam loss in regions of large dispersion. Little is known about the exact details of backgrounds due to transmission of large energy spreads through the FFTB, but it is prudent to keep the energy spread $\sigma_{E} / E$ to as low a value as reasonable. Since the dispersion through the IP1 area is negligible, energy spread is not a concern in regards to the beam focusing nor potential background generation in the vicinity of the undulator.

E-164 was run in March, 2003 with a nominal beam current of $1 \times 10^{10}$ electrons per bunch and measured emittances of $\gamma \epsilon_{x}=3.6 \times 10^{-5} \mathrm{~m}-\mathrm{rad}$ and $\gamma \epsilon_{y}=0.4 \times 10^{-5} \mathrm{~m}$-rad. Coupling in the damping ring would bring these emittances to $\gamma \epsilon_{x}=\gamma \epsilon_{y}=2 \times 10^{-5} \mathrm{~m}-\mathrm{rad}$. The energy spread of the E-164 beam was about $0.6 \%$ due to the $100-\mu \mathrm{m}$ rms bunch length. A more typical bunch length of $500 \mu \mathrm{m}$ rms would have an rms energy spread of less than $0.2 \%$. The longer bunch length results in increased transverse wakefields, which are not 
expected to be problematic at the requested beam current of $1 \times 10^{10}$ electrons per bunch.

Multiple accesses to the FFTB housing will be required after the beam has been turned on. These accesses are to install the undulator after initial beam setup and for detector background shielding adjustments. During access, the $50-\mathrm{GeV}$ beam is put onto the tuneup dump in the beam switchyard. Additionally, all power supplies and magnets remain energized during an FFTB entry. These two features, along with existing FFTB beam steering feedback systems, ensure rapid beam recovery after an access.

In summary, the requested beam and optics requirements are within the design specifications of the linac and FFTB. Furthermore, these criteria are less stringent than recent (March, 2003) operating experience.

\subsubsection{Synchrotron Radiation Background}

To avoid noise in the detectors from synchrotron radiation by electrons in upstream beam transport magnets and in the dumpline magnets, a pair of soft bends is included in the electron beamline just before and after the undulator (devices $\mathrm{HSB}_{1}$ and $\mathrm{HSB}_{2}$ in Fig. 18). These bends have the same polarity and give a vertical downward kick to the electron beam. This is similar to the geometry that was used successfully in experiment E-144 [60], albeit only one set of "hard" soft bends are used for E-166 whereas E-144 used both "hard" soft bends and "soft" soft bends. Table 7 lists expected photon parameters from the undulator and bend magnets in the immediate vicinity of the experiment. The expected flux from the undulator is significantly higher in photon energy and number. In Table $7, \hbar \omega_{c}$ is the critical energy of the synchrotron radiation emitted by the bends; $\Delta L(3 / \gamma)$ is the length of bend required to produce a $3 / \gamma$ angular deflection in the beam; and the $\Delta N_{\gamma}$ and $\Delta P_{B}$ are the radiated flux and power from the bends for the $\Delta L(3 / \gamma)$ length segment of bend.

\subsubsection{Collimators}

Fig. 18 shows two aperture-limiting collimators for the experiment, $\mathrm{A}_{u}$, which protects the undulator from possible mis-steering of the primary electron beam, and $\mathrm{A}_{t}$, which defines the photon beam used to create the polarized positrons. These devices are 30 -cm-long $(\sim 20$ rad. len.) cylinders of copper with a $0.85-\mathrm{mm}$ ID through hole for electron beam transmission in $\mathrm{A}_{u}$, while collimator $\mathrm{A}_{t}$ has a $3-\mathrm{mm}$ ID aperture for photon beam transmission.

Collimator $\mathrm{A}_{u}$ is water cooled because of the possibility of primary beam interception; collimator $\mathrm{A}_{t}$ does not require water cooling.

$A_{u}$ is required to protect the undulator assembly from being hit head-on by the primary electron beam. With $\mathrm{A}_{u}$, failure of the soft bends could result in a glancing incidence of the electron beam on the undulator. Preliminary calculations indicate that such interception would not damage the undulator in a single shot; protection ion chambers located at the undulator will cause the beam to be turned off after detection of a single shot fault.

Collimator $A_{t}$ is located just upstream of the undulator-photon conversion target, and serves to limit extraneous halo (both photons and charged particles) from entering into the detector region of the experiment. 
Table 7: Photon flux from the helical undulator. For comparison, parameters of the synchrotron radiation flux from the dump magnet $\mathrm{D}_{1}$ and the hard-soft bends $\mathrm{HSB}_{1}$ and $\mathrm{HSB}_{2}$ are also listed.

\begin{tabular}{|l|c|c|c|c|}
\hline \hline Parameter & Units & Undulator & $\mathrm{D}_{1}$ Bend & HS Bend \\
\hline \hline$E_{e}$ & $\mathrm{GeV}$ & 50 & 50 & 50 \\
\hline$n_{e}$ & $\times 10^{10} e^{-}$ & 1 & 1 & 1 \\
\hline$f_{\mathrm{rep}}$ & $\mathrm{Hz}$ & 30 & $\mathrm{dc}$ & $\mathrm{dc}$ \\
\hline$P_{e}$ & $\mathrm{~kW}$ & 2.4 & 2.4 & 2.4 \\
\hline$B_{0}$ & $\mathrm{kG}$ & 7.58 & 4.45 & 0.660 \\
\hline$K$ & - & 0.17 & - & - \\
\hline$d N_{\gamma} / d L$ & $\mathrm{photons} / \mathrm{m} / e^{-}$ & 0.37 & 2.75 & 0.41 \\
\hline $\mathrm{E}_{c 10}\left(\mathrm{E}_{c r i t}\right)$ & $\mathrm{MeV}$ & 9.62 & $(0.739)$ & $(0.110)$ \\
\hline$E_{\text {avg }}$ & $\mathrm{MeV}$ & 4.81 & 0.228 & 0.034 \\
\hline$d P_{u, B} / d L$ & $\mathrm{~mW} / \mathrm{m}$ & 87 & 30 & 0.7 \\
\hline$L_{u}(\Delta L(3 / \gamma))$ & $\mathrm{m}$ & 1 & $(0.01)$ & $(0.08)$ \\
\hline$\Delta N_{\gamma}$ & $\mathrm{photons} / \mathrm{s}$ & $1.1 \times 10^{11}$ & $9.5 \times 10^{9}$ & $9.5 \times 10^{9}$ \\
\hline$\Delta P_{u}\left(\Delta P_{B}\right)$ & $\mathrm{mW}$ & 87 & $(0.35)$ & $(0.05)$ \\
\hline \hline
\end{tabular}

\subsubsection{Alignment}

Absolute component alignment tolerances of $100 \mu \mathrm{m}$ ( $\mathrm{rms}$ ) in the transverse dimensions for the beam-line devices are required for the experiment. Collimator $A_{u}$ is rigidly mounted with the undulator to prevent a relative misalignment between the collimator and undulator. With the exception of the photon collimator, $\mathrm{A}_{t}$, none of the devices requires remote mover capability.

Because of the long lever arm $(\sim 35 \mathrm{~m})$ from the end of the undulator to the measurement area, remote movers for $A_{t}$ are incorporated into the design. The 100- $\mu \mathrm{m}$ tolerance does, however, require consideration in the design of various supports and has been taken into account. As expected, the tolerances along the beamline are very loose and are essentially set by what is required to match up and seal the vacuum chambers. 


\subsubsection{Instrumentation}

A variety of beam-line instrumentation is shown in the layout (Fig. 18). In addition to their role during beam set up, the profile monitors will be used to monitor the beam quality over the duration of the experiment.

Three beam-position monitors (BPM's) will be used in the automated beam-steering feedback to keep the beam away from the undulator and directed onto the dump.

A beam-current toroid (Toro) is used to measure the electron current on a pulse-to-pulse basis with an absolute accuracy of a few percent and a relative accuracy of a few tenths of a percent.

Four transverse beam-profile monitors (OTR, WS, $\mathrm{PR}_{d}$, and $\mathrm{PR}_{t}$ ) are shown. The OTR and WS are used in the initial optical set up of the beamline to adjust to the requisite beam size through the undulator. Monitor $\mathrm{PR}_{t}$ has been included in front of collimator $\mathrm{A}_{t}$ for observation of the photon beam. $\mathrm{PR}_{d}$ is a fixed position dumpline screen used for observing the electron beam as it enters the dump. The profile monitors OTR and $\mathrm{PR}_{t}$ are invasive monitors. Wire scanner WS provides non-invasive beam-size monitoring; however, backgrounds in the detector are likely to increase when WS is scanned through the beam.

So-called LIONS (long ion chambers) are located along the beamline wall and are used to detect secondaries caused by possible beam interception. A discrete protection ion chamber will be installed next to the undulator to detect beam loss in the undulator.

The precision and accuracy of the required instrumentation does not exceed the normal performance of the standard FFTB equipment. All of the beam-line hardware (power supplies and instrumentation) will be controlled and monitored through the existing SLAC Control System.

\subsection{The Undulator}

The helical undulator is $1 \mathrm{~m}$ long with a period $\lambda_{u}$ of $2.4 \mathrm{~mm}$ [61]. It consists of a 0.6 mm-diameter copper wire bifilar helix, wound on a 1.068-mm-O.D., stainless-steel support tube; the I.D. of the tube is $0.889 \mathrm{~mm}$. The undulator I.D. is thus \pm 11 times the rms beam size of $40 \mu \mathrm{m}$ (see Section 3.2.2).

Fig. 20 shows a 23 -cm-long prototype model built to test the winding procedure, support constraints, and voltage handling capability of the device [61]. As shown in the figure, three G10 rods and rings hold the helical coil in place.

The on-axis field in the undulator is $0.76 \mathrm{~T}$ for a $2300-\mathrm{A}$ excitation, resulting in an undulator parameter of $K=0.17$ (see eq. (5)). The presence of the stainless-steel support tube reduces the field by $<3 \%$.

Fig. 21 shows a schematic of the undulator configuration and the associated pulse-forming network. Fig. 22 shows the undulator vacuum vessel with the power supply connections entering at the center of the envelope. Modeling of the undulator has been done using MERMAID [62].

For a $30-\mu$ s-long current pulse, the temperature rise is about $3^{\circ} \mathrm{C} /$ pulse and the average power dissipation for $30-\mathrm{Hz}$ operation is about $260 \mathrm{~W}$. The undulator is immersed in an oil 

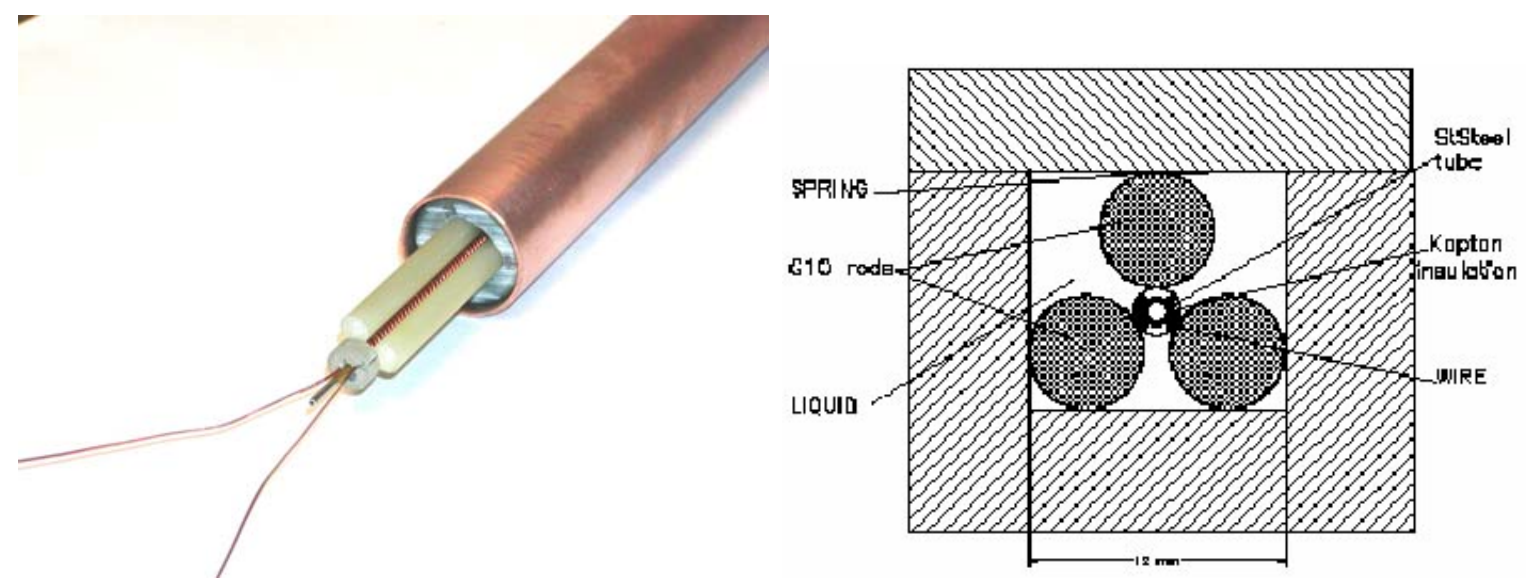

Figure 20: Left: One end of a 23-cm-long prototype of the helical undulator. Right: Cross section of the undulator. From [61].

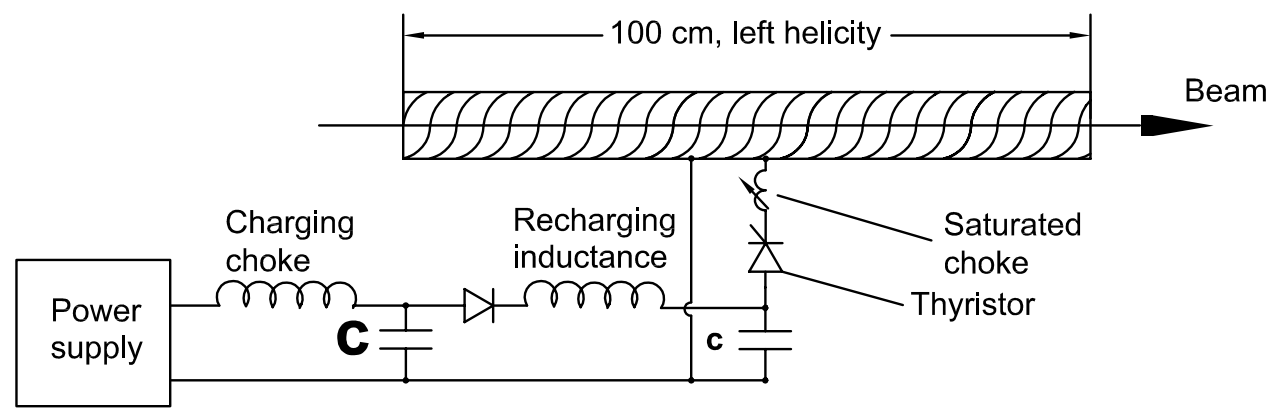

Figure 21: Schematic representation of the undulator with pulsing circuit. From [61].

bath for cooling. A water cooled heat exchanger loop is required to remove the heat from the oil.

Table 8 lists various undulator system parameters, and Table 7 lists parameters of the photon beam that emerges from the undulator.

\subsection{The Photon Polarimeter}

The concept of the photon polarimeter has been introduced in sec. 2.4 and sketched in Fig. 12. The basic components are a magnetized iron absorber and a detector that measures the photons that penetrate through the absorber.

On reversing the sign of the magnetization of the absorber, an asymmetry $\delta$ is measured in the rate of transmitted photons, which is the product of the photon polarization $P_{\gamma}$ (and the polarization $P_{e^{-}}$of the electrons in the iron) and the analyzing power $A_{\gamma}$ of eq. (16).

The implementation of the photon polarimeter for E-166 is sketched in Fig. 19. The photon polarimeter will include two types of photon detectors, a total absorption calorimeter and a Čerenkov detector. 


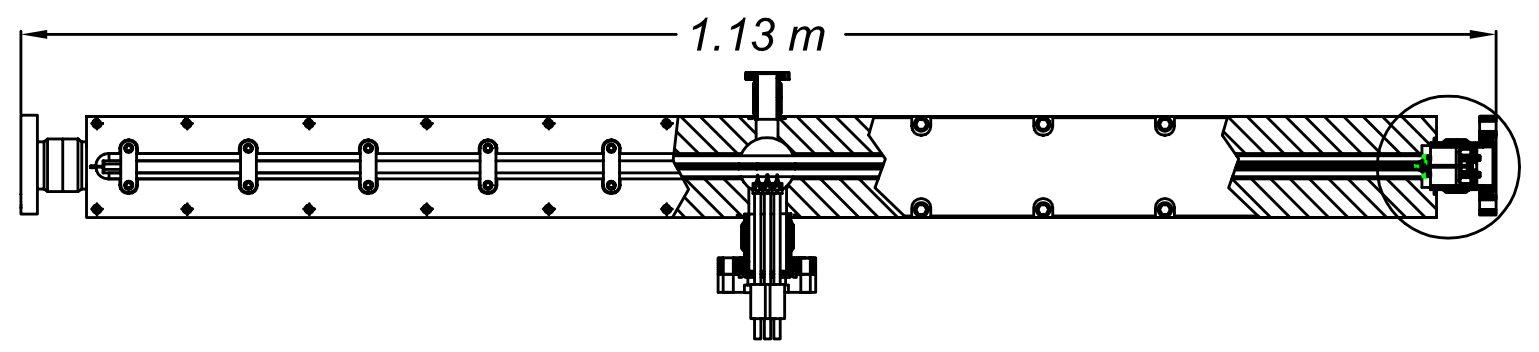

Figure 22: The 1-m-long helical undulator is mounted in a 1.13-m-long vacuum vessel; the power supply feed-through is located at the middle of the vessel. From [61].

Table 8: Parameters of the helical undulator system.

\begin{tabular}{|l|c|c|}
\hline \hline Parameter & Units & Value \\
\hline \hline Number of Undulators & - & 1 \\
\hline Length & $\mathrm{m}$ & 1.0 \\
\hline Inner Diameter & $\mathrm{mm}$ & 0.89 \\
\hline Period & $\mathrm{mm}$ & 2.4 \\
\hline Field & $\mathrm{kG}$ & 7.6 \\
\hline$K$ Undulator Parameter & - & 0.17 \\
\hline Current & $\mathrm{Amps}$ & 2300 \\
\hline Peak Voltage & $\mathrm{Volts}$ & 540 \\
\hline Pulse Width & $\mu \mathrm{s}$ & 30 \\
\hline Inductance & $H$ & $0.9 \times 10^{-6}$ \\
\hline Wire Type & - & $\mathrm{Cu}$ \\
\hline Wire Diameter & $\mathrm{mm}$ & 0.6 \\
\hline Resistance & $\Omega$ & 0.110 \\
\hline Repetition Rate & $\mathrm{Hz}$ & 30 \\
\hline Power Dissipation & $\mathrm{W}$ & 260 \\
\hline$\Delta T /$ pulse & ${ }^{0} \mathrm{C}$ & 2.7 \\
\hline \hline
\end{tabular}




\subsubsection{Magnetized Iron Absorber}

In sec. 2.4 it is shown that the optimal length of the magnetized iron absorber is about $8 \mathrm{~cm}$ when measuring the polarization of photons with energies of a few MeV. However, the iron absorber also intercepts some of the synchrotron radiation, whose critical energy is 110 $\mathrm{keV}$ (Table 7), from the soft bend magnets that surround the undulator. To minimize the transmission of this background radiation into the photon detector, a $15 \mathrm{~cm}$ long magnetized iron absorber will be used.

The probability of transmission of photons through the 15-cm-thick magnetized iron absorber is shown in Fig. 23.

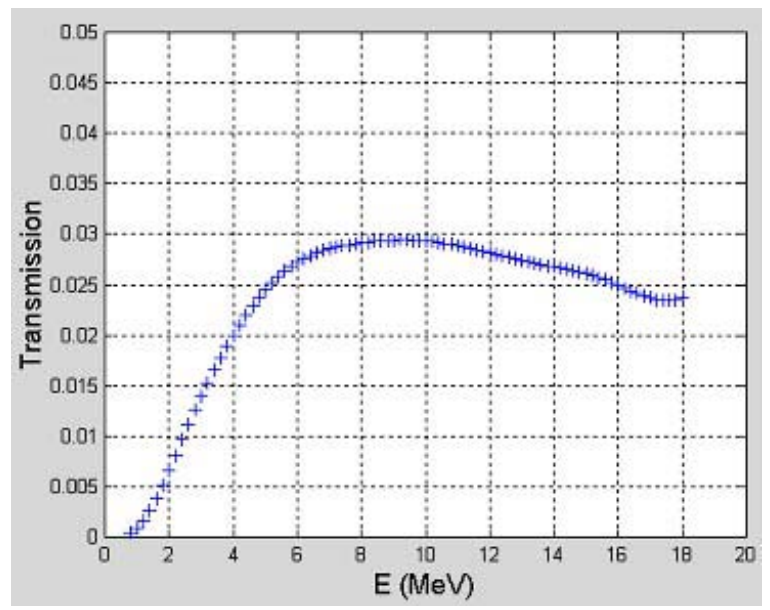

Figure 23: The probability of transmission of a photon through $15 \mathrm{~cm}$ of iron, as a function of the photon energy.

\subsubsection{Silicon-Tungsten Calorimeter}

The total absorption calorimeter for the transmitted photons is a silicon-tungsten sampling calorimeter, similar to that employed in experiment E-144 [60]. As shown in Fig. 24, this device consists of 20 plates of tungsten, each $1 \mathrm{rad}$. len. thick, separated by silicon detectors in the form of a $4 \times 4$ array of pads, each $1.6 \times 1.6 \mathrm{~cm}^{2}$ in area. The pads are read out in longitudinal groups of 5 , for a total of 64 readout channels. The resulting transverse and longitudinal segmentation of the calorimeter will permit confirmation that the energy deposited in the calorimeter has the profile expected from the signal of undulator photons, rather than that of possible backgrounds of scattered electrons and photons.

The resolution of a similar sampling calorimeter has been measured to be [60]

$$
\sigma_{\mathcal{E}}^{2}=(0.19)^{2} \mathcal{E}+(0.4)^{2}
$$

where $\mathcal{E}$ is the electron energy in $\mathrm{GeV}$. For a pulse of $10^{10}$ electrons, about $100 \mathrm{TeV}$ of energy in the undulator photons that reach the calorimeter is expected, leading to a relative error on that energy of only $0.06 \%$. 


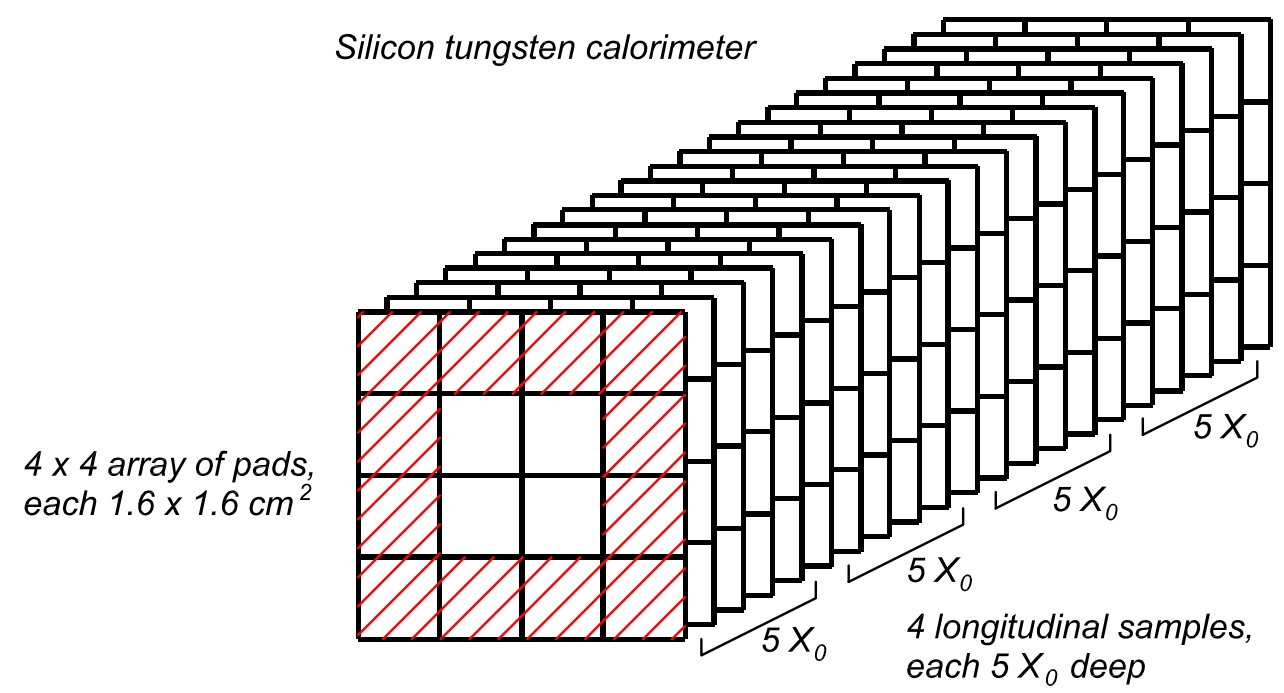

Figure 24: The silicon tungsten calorimeter consists of 20 longitudinal samples of $1 X_{0}$ each, grouped into 4 segments of $5 X_{0}$ each. The transverse sampling is via a $4 \times 4$ array of pads, each $1.6 \times 1.6 \mathrm{~cm}^{2}$ each.

\subsubsection{Aerogel Flux Counters}

A complementary measurement of the transmitted photon flux will be made with a pair of aerogel Cerenkov counters with index of refraction $n=1.007$. This extremely low-index material is available from the BELLE experiment. The two flux counters are deployed before and after the magnetized iron absorber, as shown in Fig. 19.

The signal in the aerogel flux counter is generated by conversion of undulator photons in the aerogel, after which electrons and positrons of energy greater than $4.3 \mathrm{MeV}$ will emit Cerenkov light. This light is observed in a photomultiplier that views the aerogel through an air light pipe, as shown in Fig. 25.

Because of their threshold energy of $4.3 \mathrm{MeV}$, the aerogel flux counters are insensitive to synchrotron radiation in the beam. Hence, a pair of aerogel flux counters that are placed upstream and downstream of the magnetized iron absorber, as shown in Fig. 19, can confirm the attenuation of this absorber on photons of energy above $5 \mathrm{MeV}$, independent of possible backgrounds of lower-energy photons.

Aerogel of index 1.007 has mass density $0.033 \mathrm{~g} / \mathrm{cm}^{3}$. The conversion probability of an undulator photons in a 2 -cm-thick slab of aerogel (nominally $0.002 \mathrm{rad}$. len.) is only about 0.0003 because of the low energy of the photons. The number of photons of energy above $5 \mathrm{MeV}$ that penetrate the iron absorber is about $3 \times 10^{7}$ per pulse of $10^{10}$ electrons, so the number of conversions is about $10^{4}$. There will be about $50 \theta_{C}^{2} \approx 5$ photoelectrons per conversion from the Cerenkov radiation in the aerogel, for a signal of about 50,000 photoelectrons per pulse in the downstream flux counter. 


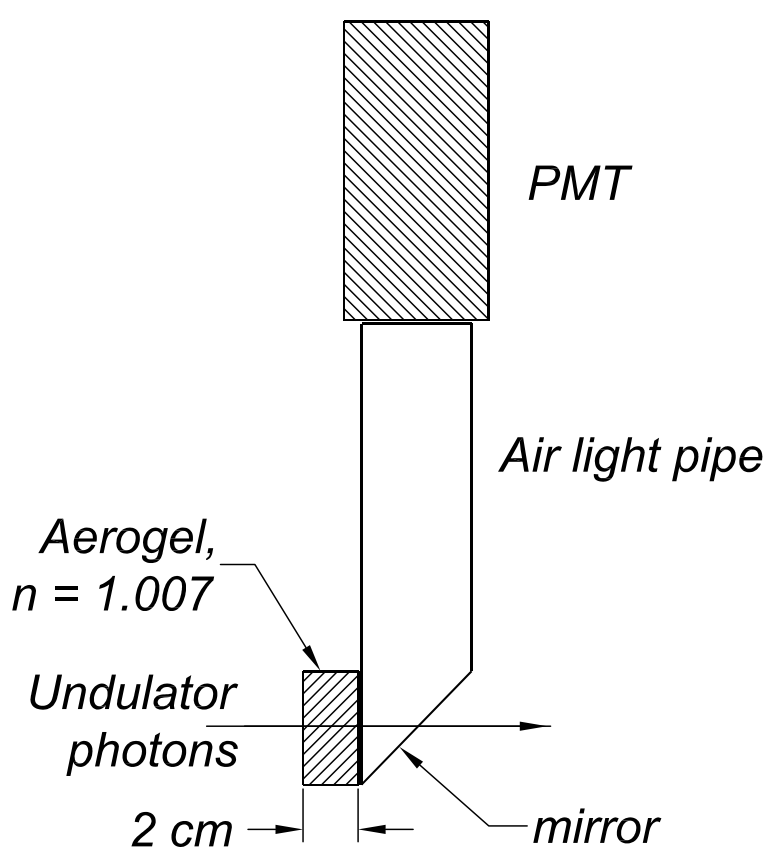

Figure 25: The photon flux counter, consisting of a 2-cm-thick block of aerogel of index $n=1.007$, viewed by a photomultiplier tube at the end of an air light pipe.

\subsection{The Positron Production Target}

For a Linear Collider the primary target material of choice is a high-strength titanium(Ti)alloy; a tungsten(W)-rhenium(Re)-alloy is considered as a backup. In comparison to the W-Re-alloy, the Ti-alloy exhibits better energy deposition characteristics and produces a somewhat higher total positron polarization. W-Re-alloy by contrast gives a higher yield and is the preferred material in conventional positron sources due to its high strength and short radiation length, $X_{0}$. The advantage of a short $X_{0}$ is less important when an external photon source is used because the emittance of the generated positrons is governed by the incident photon beam size rather by the development of a cascade shower (as in a conventional source).

If W-Re-alloy is substituted for $\mathrm{Ti}$ as the target material, the resultant positron fluxes are qualitatively very similar to the those for Ti. For W-Re-alloy targets, the raw yield is higher, the polarization is lower, and the emittance is lower. The energy spectra are very similar. Table 9 lists positron properties for both $\mathrm{Ti}$ and W-25\%Re targets of different lengths when irradiated by the E-166 photon beam with a transverse Gaussian spatial distribution of 450 $\mu \mathrm{m}$ rms. In Table 9, the raw yield is the ratio of the total number of emitted positrons to the number of incident photons; the raw polarization is the mean longitudinal positron polarization average of all positrons; $\sigma_{x \prime}$ is the standard deviation of the horizontal angular distribution of positrons; and the transverse emittance is the rms horizontal emittance normalized by the (mean positron energy) $/ \mathrm{mc}^{2}$. The mean positron energy is about $3.9 \mathrm{MeV}$ for $\mathrm{Ti}$ and $4.0 \mathrm{MeV}$ for $\mathrm{W}-25 \% \mathrm{Re}$; the standard deviation of the energy spectra is $2 \mathrm{MeV}$ 
for all cases.

Table 9: Properties of positrons generated in $\mathrm{Ti}$ and $\mathrm{W}$-Re alloy targets, assuming incident photons from a helical undulator with $K=0.17$, firstharmonic cutoff energy of $E_{c 10}=9.62 \mathrm{MeV}$, and an rms spot size of $450 \mu \mathrm{m}$.

\begin{tabular}{|l|c|c|c|c|c|}
\hline \hline Material & Thickness & Raw Yield & Raw Polarization & $\sigma_{x \prime}$ & Transv. Emit. \\
\hline \hline $\mathrm{Ti}$ & (rad. len.) & $(\%)$ & $(\%)$ & $(\mathrm{rad})$ & $(\pi$ m-rad $)$ \\
\hline $\mathrm{Ti}$ & 0.05 & 0.34 & 42 & 1.1 & 0.005 \\
\hline $\mathrm{Ti}$ & 0.1 & 0.46 & 48 & 1.4 & 0.008 \\
\hline $\mathrm{Ti}$ & 0.25 & 0.51 & 52 & 1.3 & 0.009 \\
\hline $\mathrm{W}-25 \% \operatorname{Re}$ & 0.05 & 0.43 & 53 & 0.7 & 0.005 \\
\hline $\mathrm{W}-25 \% \operatorname{Re}$ & 0.1 & 0.67 & 36 & 1.3 & 0.005 \\
\hline $\mathrm{W}-25 \% \operatorname{Re}$ & 0.25 & 0.93 & 41 & 1.2 & 0.005 \\
\hline $\mathrm{W}-25 \% \operatorname{Re}$ & 0.5 & 0.93 & 51 & 1.5 & 0.006 \\
\hline \hline
\end{tabular}

E-166 will start with $0.5 \mathrm{rad}$. len. of Ti-alloy and scan through Ti targets of different thicknesses. As time permits, the experiment will investigate the production of polarized positrons using a W-25\%Re target.

\subsection{The Positron Polarimeter}

As discussed in sec. 2.5, the measurement of positron polarization is to be made by first transferring the polarization to photons, and then using a photon-transmission polarimeter. Measurements of the asymmetry $\delta$ in the rate of transmitted photons can be related to the positron polarization $P_{e^{+}}$via a calculable analyzing power $A_{e^{+}}$, as discussed in sec. 2.5.

The layout of the positron polarimeter has been shown in Fig. 19, and is shown again in Fig. 26. A double $90^{\circ}$-bend magnet transports the positrons to the reconversion target $(0.5$ rad. len. of tungsten). The photons that emerge from the target are then incident on an 7.5cm-long magnetized iron absorber. The photons that are transmitted through the absorber are detected in a CsI array. The latter device was chosen, rather than a Si-W calorimeter, because the typical energy of photons reaching the detector in the positron polarimeter is only about $1 \mathrm{MeV}$; the energy resolution of a CsI calorimeter for such energies is about 2.5\%, compared to $20 \%$ for a Si-W device.

Some of the parameters of the positron polarimeter are summarized in Table 10. 


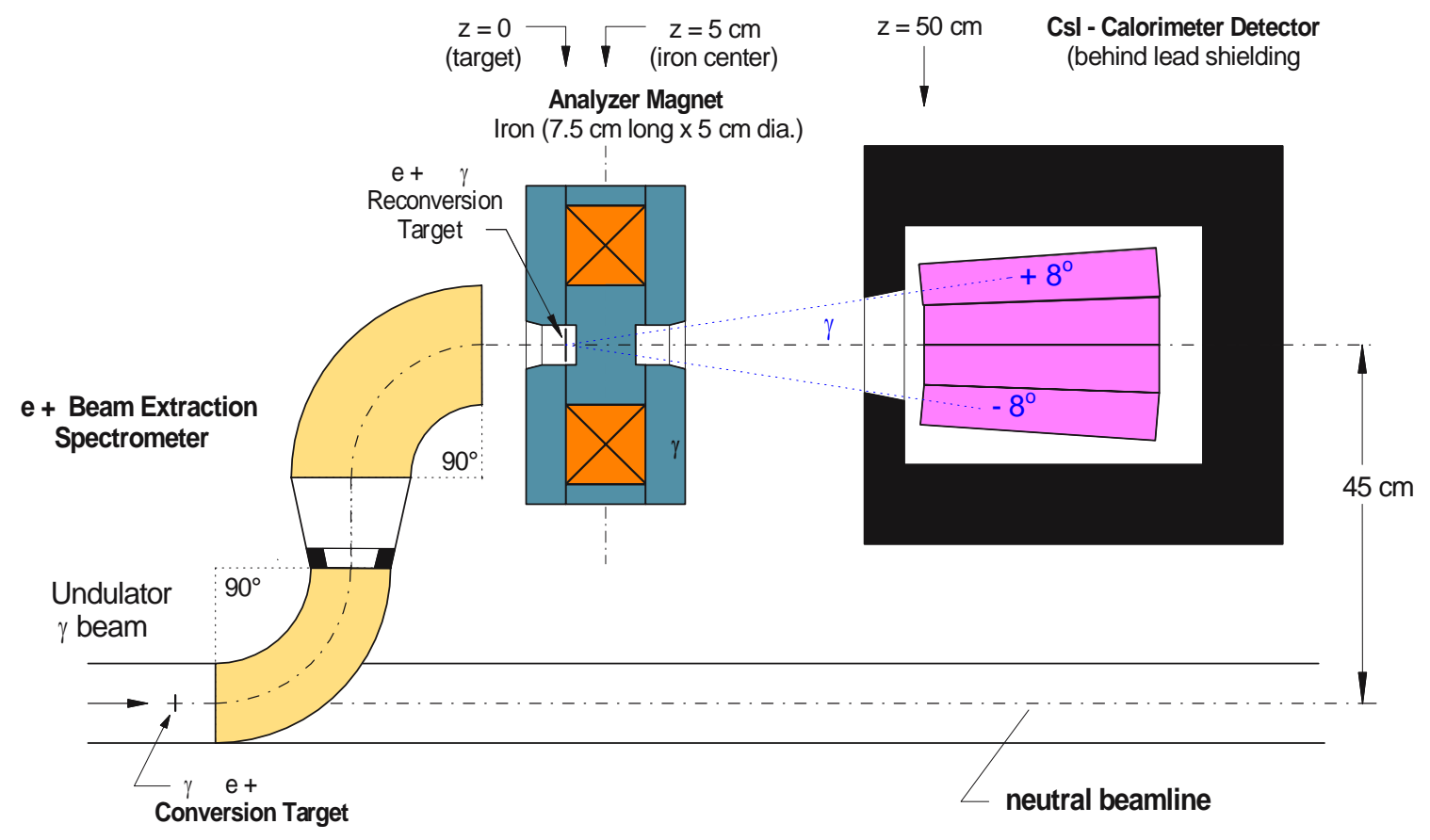

Figure 26: Layout of the positron polarimeter.

Table 10: Parameters of the positron polarimeter.

\begin{tabular}{lc}
\hline \hline e+ Energy spread & $\pm 20 \%$ \\
W Reconverter thickness & $0.175 \mathrm{~cm}$ \\
Fe absorber thickness & $7.5 \mathrm{~cm}$ \\
Fe absorber radius & $2.5 \mathrm{~cm}$ \\
CsI Detector aperture & $7 \mathrm{~cm}($ radius $)$ \\
Distance between reconverter and iron & $1.25 \mathrm{~cm}$ \\
Distance between iron and CsI detector & $41.25 \mathrm{~cm}$ \\
\hline \hline
\end{tabular}

\subsubsection{The Positron Transport System}

The positron polarimeter includes a transport system between the target in which the positrons are created and the reconversion target in which their polarization is transferred to photons. This transport selects positrons within a momentum band of about $\pm 20 \%$, as shown in Fig. 27, while rejecting electrons, and minimizing the transport of background photons to the reconversion target.

The suppression of background photons, generated in the primary production target or when positrons and electrons hit the walls of the spectrometer vacuum chamber, is the prin- 


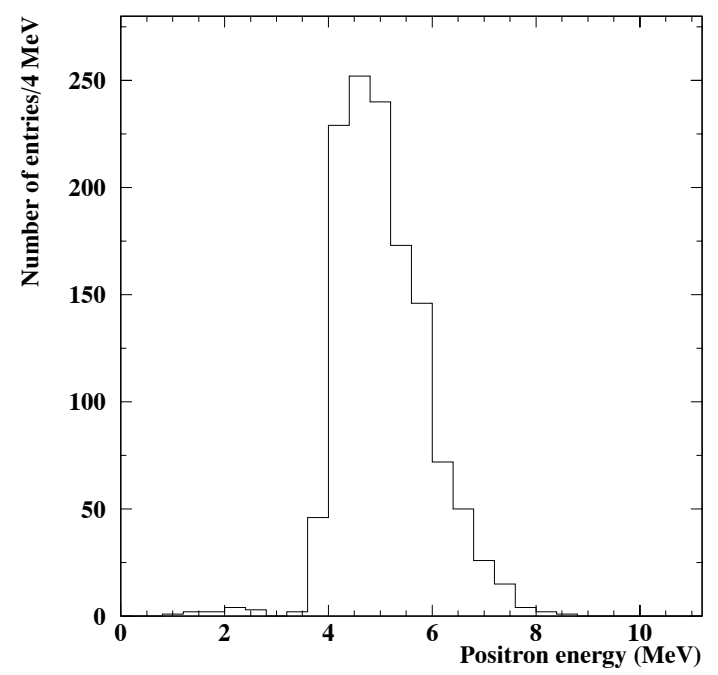

Figure 27: Momentum acceptance of the positron polarimeter when tuned to a central momentum of $5 \mathrm{MeV} / c$.

cipal design criterion of the beam transport, because such background photons outnumber positrons by 200 to 1 just after the production target. A double $90^{\circ}$ system of bend magnets accomplishes this task by providing relatively dispersion-free transport of the positrons through a "dogleg" and onto a beamline $45 \mathrm{~cm}$ offset from the undulator photon beamline.

A set of jaws with variable aperture is placed between the two $90^{\circ}$ bend magnets to provide the option for high resolution momentum scans, and for background studies with the jaws closed.

Figure 28 shows how background photons from the positron production target, or from positrons and electrons that intercept the walls of spectrometer vacuum chamber, can reach the reconversion target only after two or more scatters. Positrons that pass through the momentum-selecting jaws are refocused onto the reconversion target by the second bend magnet, as shown in Fig. 29.

Figure 30 shows the result of a GEANT simulation which indicates that the transport system can deliver about $2 \%$ of all positrons produced at the primary target onto the reconversion target, for central momenta of the transport around $5 \mathrm{MeV}$. Around $0.5 \%$ of the positrons which arrive at the reconversion target are registered at the CsI-detector. Figure 31 shows the background fraction expected at the CsI detector as a function of the mean energy of the signal positrons. For momenta above around $5 \mathrm{MeV}$ this fraction is below $5 \%$.

\subsubsection{The Magnetized Iron Absorber}

Magnetized iron represents a convenient polarized electron target. Of the 26 electrons in the iron atom only 2 can be spin polarized, giving an overall polarization of $2 / 26 \simeq 0.07$ or $7 \%$ at saturation. Quantitatively the electron polarization is given by 


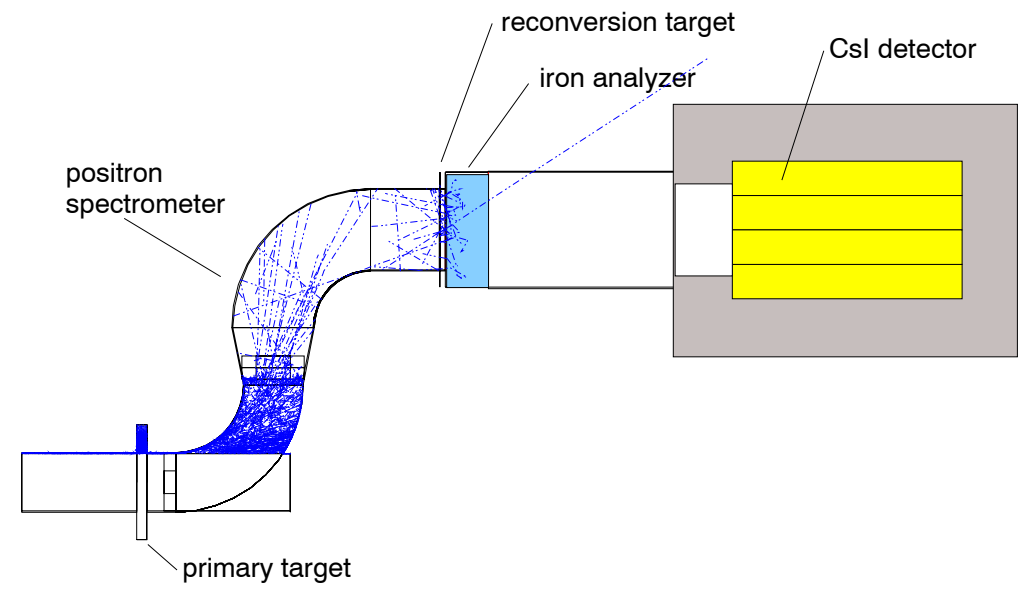

Figure 28: Examples of trajectories of photons in the upstream part of the positron polarimeter.

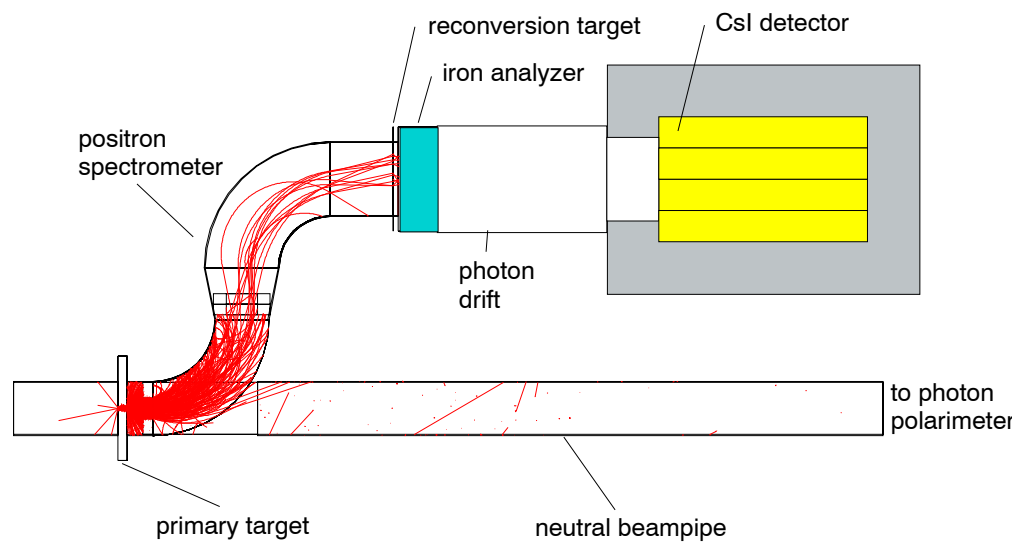

Figure 29: Examples of trajectories of positrons in the upstream part of the positron polarimeter.

$$
P_{e}=2 \cdot \frac{g^{\prime}-1}{g^{\prime}} \cdot \frac{M}{n \mu_{B}}
$$

where $M=\left(B-B_{o}\right) / \mu_{o}$ is the magnetization, $\mathrm{n}$ is the number of electrons per unit volume, $\mu_{B}$ is the Bohr magneton, and $g^{\prime}$ is the gyromagnetic ratio which is known from Einstein-de Haas type experiments [63].

In order to attain the maximum possible polarization the iron must be magnetized to saturation in the volume where the photons traverse the material. Fig. 32 shows the field distribution of a suitable iron and coil geometry that we have calculated and optimized with the Opera-2d software from Vector Graphics.

The water-cooled coil has 360 turns and is operated at 100 Amperes. The volume of interest is $7.5 \mathrm{~cm}$ long $\mathrm{x} 5 \mathrm{~cm}$ diameter and will be fully in saturation, except for small regions at the entrance and exit faces. 


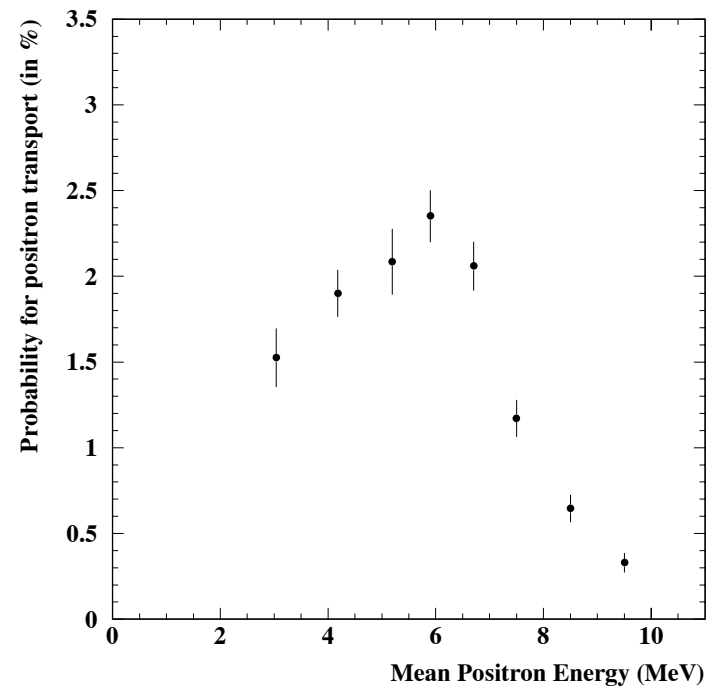

Figure 30: The fraction, in per cent, of all positrons produced at the primary target that reach the reconversion target in the positron polarimeter, as a function of the central momentum of the polarimeter.

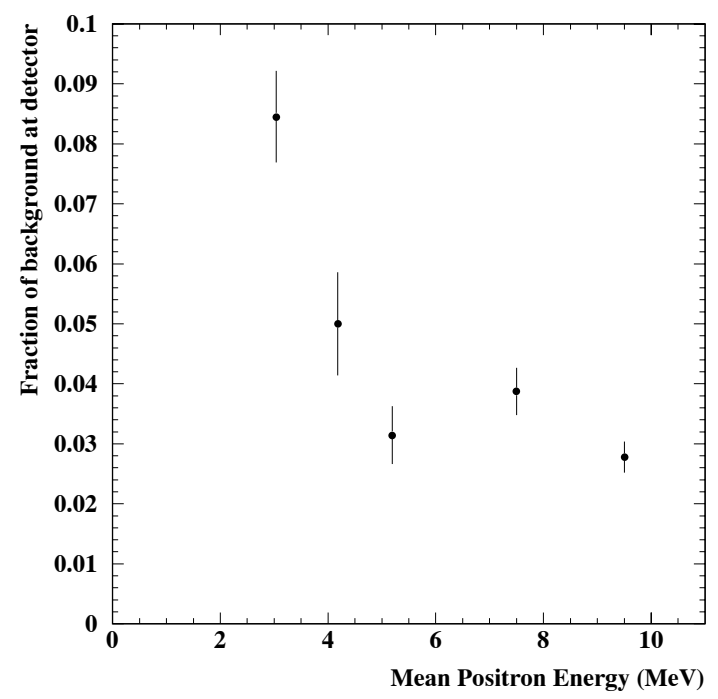

Figure 31: Fraction of background events in the CsI detector, as a function of the central momentum of the polarimeter.

\subsubsection{The CsI Calorimeter}

The calorimeter for the detection of the photons radiated from the polarized positrons will be based on the technology developed for the BaBar experiment [64]. It will be a crystal calorimeter with thallium-doped CsI crystals of typical dimensions: front face $4.7 \times 4.7 \mathrm{~cm}^{2}$, 


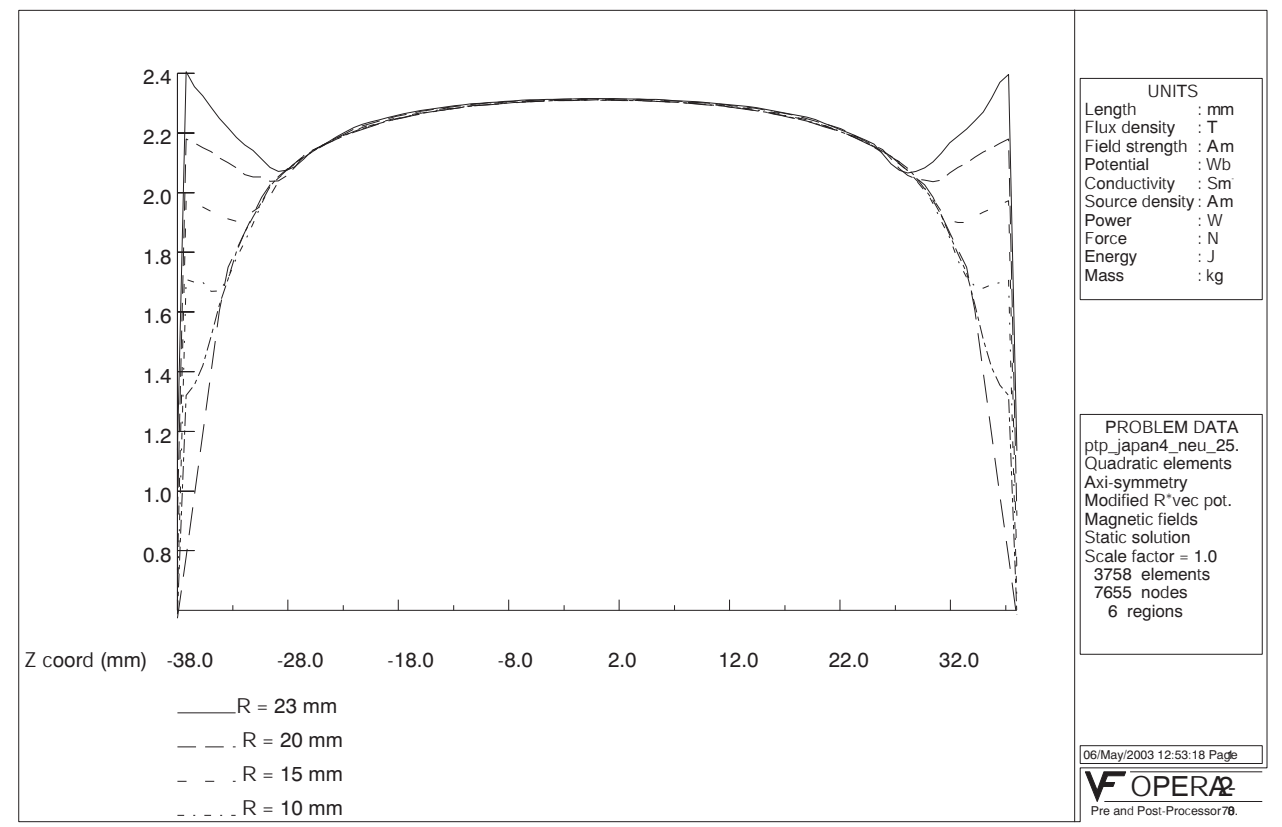

Figure 32: Calculated field map along the axis of the magnetized iron absorber. The vertical scale is the field in Tesla. The lowermost curve (unlabeled) is for the field along the axis $(R=0)$.

rear face $6.0 \times 6.2 \mathrm{~cm}^{2}$, and length $30 \mathrm{~cm}$. Sixteen crystals will be stacked in a $4 \times 4$ array inside a light-tight box. The box will be placed inside a lead housing to protect the calorimeter against background radiation. At the front of the housing there is a circular opening that defines the acceptance of the calorimeter.

The scintillation of the crystals is detected from the rear end by two large area photo diodes (Hamamatsu S2744-08, active area $2 \mathrm{~cm}^{2}$ each). A preamplifier is mounted directly behind the diodes, from where the signal is transferred to ADC's. We plan to use the BaBar preamps, but conventional ADC's. BaBar has achieved light yields between 5000 and 10000 photo electrons per $\mathrm{MeV}$ with two photo diodes.

The two diodes are glued onto a thin glass plate to improve the optical matching to the CsI. The glass plate is then pressed against the back surface of the CsI by two springs. Optical grease will be used to improve the light coupling. The two diodes, the glass plate and the preamp will be mounted inside an aluminum housing (see Fig. 33. A cooling loop will run along the walls to remove the heat created by the preamps. Flourinert will be used as coolant to protect the crystals in case of leakage.

The 16 CsI crystals needed for the present experiment will be provided by SLAC (group E) out of the pool of spare crystals for the BaBar experiment,purchased from SLAC stores. These crystals have photodiodes already mounted onto the coupling plates. A set of 32 preamps and housing are also available.

\subsection{Data-Acquisition System}

For the measurements forseen by E-166, several parameters need to be set and data from 


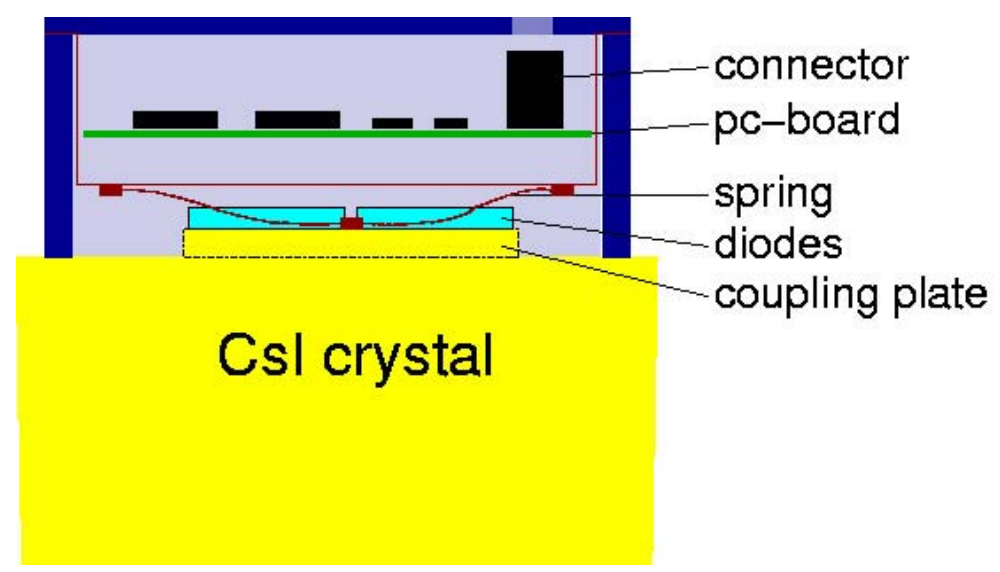

Figure 33: Mounting of the readout electronics on the end of a CsI crystal.

the apparatus need to be recorded. Table 11 shows some of these quantities.

The data acquisition and control (DAQ) system has to record and display these data, and possibly set other parameters. As the channel count and trigger frequency $(30 \mathrm{~Hz})$ are rather modest, an existing SLAC-provided PC running LabView is sufficient. This PC is interfaced to a CAMAC crate housing the front-end electronics (ADC's and such) with a GPIB card; other in- and output may be done with a PCI I/O board also installed in the PC.

The DAQ system is synchronized to the SLC/FFTB timing through a SLC PDU (Programmable Delay Unit), which provides trigger pulses with a programmable delay, either generated only when beam is present ('TRIG'), or always present ('TRBR'). For data-taking with beam, trigger pulses are used to make gates pulses for the ADC conversions; after a suitable delay the PC will read out the digitized data from the CAMAC crate, display them, and write them to disk on the PC. The data will then be transferred to a SLAC Unix storage for later off-line analysis.

A separate LabView process can read out a 'Smart Analog Module' (SAM) also present in the CAMAC crate, asynchronously with the beam; the SAM collects beam data (energy,positions, flux) from the SLAC Control System. 
Table 11: Elements of the data-acquisition system. SLC = SLAC Control System, DAQ = E-166 Data Acquisition System.

\begin{tabular}{|l|l|c|c|c|}
\hline \hline Device & $\begin{array}{l}\text { Quantity } \\
\text { Measured }\end{array}$ & $\begin{array}{c}\text { No. of } \\
\text { Channels }\end{array}$ & $\begin{array}{c}\text { Recorded by } \\
\text { / set in }\end{array}$ & $\begin{array}{c}\text { Pulsed (P) } \\
\text { or Slow (S) }\end{array}$ \\
\hline \hline $\begin{array}{l}\text { Cerenkov/ } \\
\text { Aerogel }\end{array}$ & Particle flux & 2 & DAQ & $\mathrm{P}$ \\
\hline Si/W Calor. & $\begin{array}{l}\text { Integrated } \\
\text { photon energy }\end{array}$ & 64 & DAQ & $\mathrm{P}$ \\
\hline CsI Calor. & $\begin{array}{l}\text { Integrated } \\
\text { photon energy }\end{array}$ & 32 & DAQ & $\mathrm{P}$ \\
\hline Iron magnet & Field direction & 2 & DAQ & $\mathrm{S}$ \\
\hline Iron magnet & Current & 2 & DAQ & $\mathrm{S}$ \\
\hline Undulator & Current & 1 & SLC & $\mathrm{S}$ \\
\hline Toroid & $e^{-}$flux & 1 & SLC & P \\
\hline & Beam energy & 1 & SLC & S \\
\hline \hline & Bunch length & 1 & SLC & S \\
\hline \hline
\end{tabular}




\section{Measurements of Photon and Positron Polarization}

The principal measurements in the proposed experiment are those of the flux and polarization of the photon beam created in the helical undulator (sec. 3.3), and those of the flux and polarization of the positrons that are obtained from the undulator photons by conversion in a thin target (sec. 3.5). The polarization measurements are made with the technique of transmission polarimetry, whose principles have been introduced in secs. 2.3-4, with apparatus described in secs. 3.4 and 3.6.

Each $50-\mathrm{GeV}$ electron is expected to produce 0.4 photons on average. In the case of the photon polarimeter, the transmission through the iron absorber is about $1 \%$. Thus, for $1 \times 10^{10}$ electrons per pulse, $4 \times 10^{9}$ photons per pulse are made, of which $4 \times 10^{7}$ photons arrive at the detector in the photon polarimeter.

For the positron studies, each photon pair-produces at a rate of about 0.005 positrons per photon in the primary target. About $2 \%$ of these positrons are transported to the reconversion target of the positron polarimeter, where roughly 1 photon per positron is regenerated. There is about a $0.25 \%$ transmission efficiency of photons from the reconversion target into the detector.

Thus, the $4 \times 10^{9}$ undulator photons per pulse result in $2 \times 10^{7}$ positrons per pulse. Of

these, $4 \times 10^{5}$ positrons are incident on the reconversion target and about $1 \times 10^{3}$ photons arrive at the detector in the positron polarimeter.

\subsection{Undulator Photons}

\subsubsection{Flux}

The flux of undulator photons of energy above 4.4 MeV is measured with the first of the two aerogel flux counters, shown in Fig. 19 and described in sec. 3.4.3. The signal in these flux counters is due to Cerenkov radiation of electrons and positrons produced in interactions of the undulator photons with a block of aerogel of index 1.007. The aerogel was chosen to be insensitive to the synchrotron radiation from the bend magnets $\mathrm{HSB}_{1}$ and $\mathrm{HSB}_{2}$ (Fig. 18), whose critical energy is $110 \mathrm{keV}$ and whose integrated photon number is comparable to that of the undulator radiation. However, only the upper half of the undulator photon energy spectrum (Fig. 6(a)) is thereby monitored.

If it proves desirable to have a confirming measure of the flux of the entire spectrum of undulator photons, the iron absorber of the photon polarimeter could be removed, and the Si-W calorimeter (sec. 3.4.2) used to measure the total energy in the photon beam.

A second aerogel flux counter, identical to the first, will be located downstream of the iron absorber in the photon polarimeter to monitor the flux of photons transmitted through that absorber. The ratio of rates in these two flux counters will be a good measure of the transmission of the photons through the iron, since the transmission coefficient is essentially flat for photons of energy 5-10 MeV, as shown in Fig. 14(b).

\subsubsection{Polarization}

The polarization of undulator photons is to be measured in a transmission polarimeter 
consisting of a 15-cm-long magnetized iron absorber followed by an aerogel flux counter and a Si-W calorimeter (sec. 3.4).

\section{Polarimetry Using the Si-W Calorimeter}

The expected spectrum and polarization of the undulator photons are shown in Figs. 6 and 7 . The energy-weighted average polarization of the undulator photons is $50 \%$ before the iron absorber, and $61 \%$ behind it.

The spectrum of photons that are transmitted through the iron absorber is shown in Fig. 34(a), and the corresponding asymmetry on reversal of the magnetization of the iron is shown in Fig. 34(b).
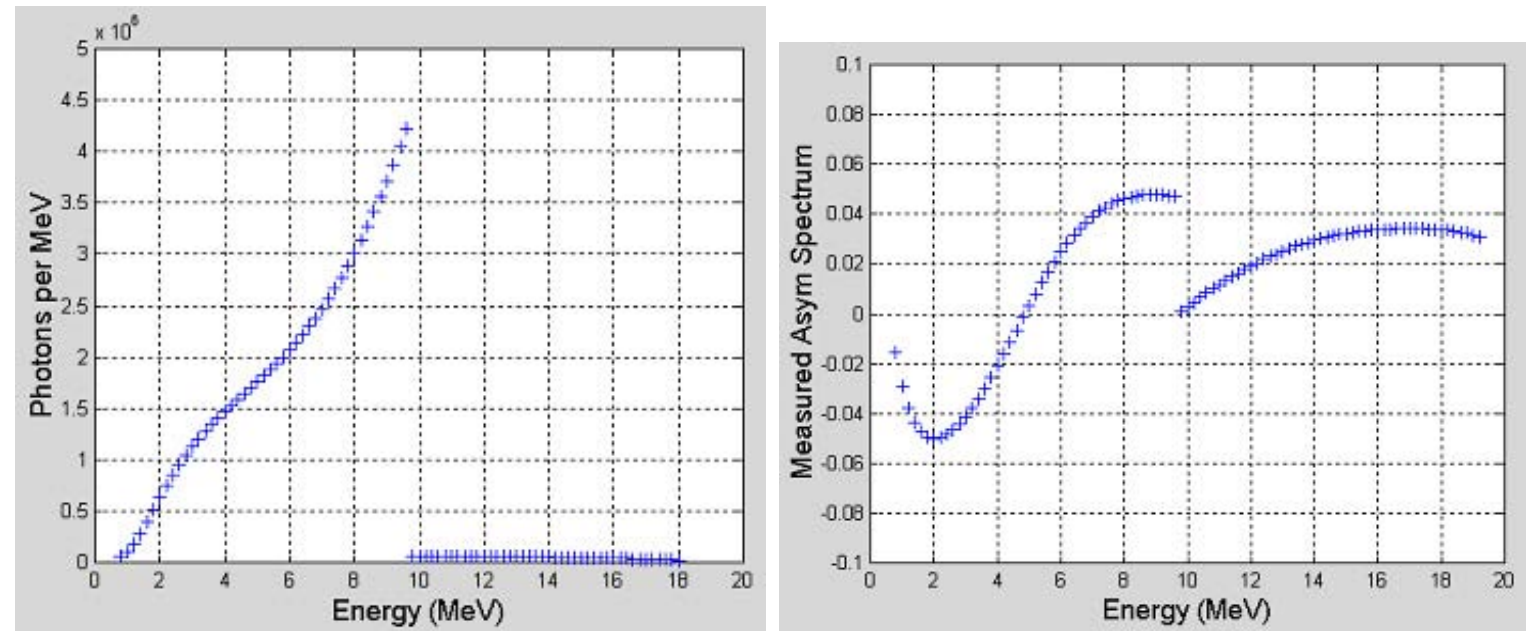

Figure 34: (a) The number spectrum of undulator photons that penetrate through the $15-\mathrm{cm}$ magnetized iron absorber. The spectrum of photons incident on the absorber is shown in Fig. 6(a). (b) The asymmetry of photons transmitted through the iron absorber on reversal of its magnetic field.

When the photons that have penetrated the 15-cm-long iron absorber are observed in the Si-W calorimeter, an energy-weighted asymmetry $\delta$ of $2.66 \%$ is expected, according to the simulations.

We interpret this result as measurement of the energy-weighted mean transmitted photon polarization, from eq. (17),

$$
\left\langle P_{\gamma}^{E}\right\rangle=\frac{\delta}{P_{e}\left\langle A_{\gamma}^{E}\right\rangle},
$$

where $P_{e} \approx 0.07$ is the net polarization of the electrons in the iron absorber, and $\left\langle A_{\gamma}^{E}\right\rangle \approx 0.62$ is the energy-weighted analyzing power. The latter is calculated in a simulation of the transmission asymmetry $\delta$ that would occur for a beam of photons of energy and polarization spectra as in Fig. 6. This gives

$$
\left\langle P_{\gamma}^{E}\right\rangle=\frac{0.0266}{0.07 \cdot 0.62}=0.61
$$




\section{Polarimetry Using the Aerogel Flux Counter}

When the second aerogel flux counter is used for photon polarimetry, it records only photons with energy above roughly $5 \mathrm{MeV}$, and with a signal size roughly independent of energy. In this case the photons are characterized by a number-weighted polarization evaluated above the Čerenkov threshold.

Table 12 gives results of a simulation of photon polarization measurements as a function of the threshold of the Čerenkov detector. For a 5-MeV Cerenkov threshold, the theoretical

number-weighted beam polarization $\left\langle P_{\gamma}^{\text {th }}\right\rangle$ of the undulator photons is $75 \%$. Only $0.84 \%$ of all undulator photons penetrate the 15-cm-long iron absorber and have energy above 5 $\mathrm{MeV}$. The asymmetry in the detector on reversal of the magnetization is $\delta=3.2 \%$ with this threshold, for which the analyzing power $\left\langle A_{\gamma}\right\rangle$ is $60 \%$.

\section{Errors}

The statistical error on the measurement of the photon polarization is, to a good approximation when the asymmetry $\delta$ is small,

$$
\Delta P_{\gamma}=\frac{1}{P_{e}\left\langle A_{\gamma}\right\rangle} \sqrt{\frac{4 N^{+} N^{-}}{N^{3}}} \approx \frac{1}{P_{e}\left\langle A_{\gamma}\right\rangle \sqrt{N}} \approx \frac{24}{\sqrt{N}},
$$

where $N=N^{+}+N^{-}$is the total number of photons observed in the detector. The approximation holds when the asymmetry $\delta$ is small, and the numbers $N^{ \pm}$of photons detected for the two signs of $P_{e}$ are nearly equal.

In an imagined data "run" that consisted of only a single pulse of each sign of the magnetization, the total number of photons that convert in the Cerenkov radiator is 36,000, assuming a detection probability of 0.0003 as discussed in Ch. 3.4.3, and the statistical error on the measurement of the photon polarization would be $12 \%$ according to eq. (23). Hence, a total of 300 pulses (10 sec at $30 \mathrm{~Hz}$ operation) would be sufficient to obtain a statistical error of $1 \%$.

The abundance of statistics is even more impressive for the Si-W calorimeter due to its $100 \%$ detection probability. Since $N^{+} \approx N^{-} \approx 3 \times 10^{7}$ per pulse, an experiment with only a single pulse of each sign of $P_{e}$ would have a statistical error on $P_{\gamma}$ of only $0.3 \%$.

The measurement of the photon polarization will be dominated by systematic uncertainty in the electron polarization $P_{e}$ and in the analyzing power $\left\langle A_{\gamma}\right\rangle$. The uncertainty in $P_{e}$ is due to uncertainty in the degree of saturation of the iron throughout the absorber, and is typically estimated at about 5\%. The uncertainty in the calculation of $A_{\gamma}$ is largely determined by uncertainty in geometric effects on the transmission of photons through the absorber; i.e., on the probability that scattered photons find their way into the detector along with unscattered photons. With careful detector design, this uncertainty should be no more than $1 \%$. 
Table 12: Simulated results of measurements of undulator photon polarization as a function of the threshold energy of the Čerenkov detector downstream of the iron absorber. Only one beam pulse is considered for each sign of the magnetization of the iron. Each of these pulses contains $N_{0}=4 \times 10^{9}$ undulator photons. The number of photons detected in the Cerenkov detector is $N_{\gamma}=$ $2\left(\epsilon_{\gamma}\right)(0.0003) N_{0}$, where 0.0003 is the probability that a photon converts in the Cerenkov radiator.

\begin{tabular}{c|c|c|c|c|c|c|}
\hline \hline $\begin{array}{c}\text { Photon } \\
\text { Threshold } \\
(\mathrm{MeV})\end{array}$ & $\begin{array}{c}\text { Photon } \\
\text { Pol. } \\
\left\langle P_{\gamma}^{\text {th }}\right\rangle(\%)\end{array}$ & $\begin{array}{c}\text { Photon } \\
\text { Transport } \\
\text { Eff. } \epsilon_{\gamma}(\%)\end{array}$ & $\begin{array}{c}\text { Photon } \\
\text { Asym. } \\
\delta(\%)\end{array}$ & $\begin{array}{c}\text { Analyzing } \\
\text { Power } \\
\left\langle A_{\gamma}\right\rangle(\%)\end{array}$ & $\begin{array}{c}N_{\gamma} \\
\text { in 2 pulses }\end{array}$ & $\begin{array}{c}2 \text { pulse } \\
\text { Abs. Error } \\
\Delta P_{\gamma}(\%)\end{array}$ \\
\hline \hline 0 & 42.1 & 2.13 & 1.9 & 64.6 & 47377 & 10 \\
1 & 42.3 & 2.13 & 1.9 & 64.5 & 47328 & 10 \\
2 & 45.4 & 2.08 & 2.0 & 63.8 & 46238 & 10 \\
3 & 53.6 & 1.97 & 2.3 & 62.9 & 43650 & 11 \\
4 & 64.6 & 1.81 & 2.8 & 61.7 & 40169 & 12 \\
5 & 75.1 & 1.64 & 3.2 & 60.3 & 36336 & 12 \\
6 & 83.6 & 1.43 & 3.4 & 58.8 & 31739 & 14 \\
7 & 89.7 & 1.16 & 3.6 & 57.3 & 25813 & 16 \\
8 & 93.1 & 0.81 & 3.6 & 56.0 & 18040 & 19 \\
9 & 92.2 & 0.39 & 3.5 & 54.7 & 8649 & 28 \\
\hline \hline
\end{tabular}

\subsection{Positrons}

\subsubsection{Flux}

A momentum scan of the positrons produced by conversion of undulator photons in the primary target can be made by temporarily removing the reconversion target and the iron absorber from the positron polarimeter (Fig. 26). The jaws between the first and second $90^{\circ}$ bend magnets will be reduced so the momentum acceptance is $\pm 5 \%$ (compared to $\pm 20 \%$ for normal operation of the positron polarimeter). Then, the acceptance of the spectrometer for positrons will be about $1 / 4$ of that shown in Fig. 30, i.e., about $0.5 \%$.

Of the $2 \times 10^{7}$ positrons produced per pulse, about $10^{5}$ would be detected during each pulse of a scan of flux vs. momentum. Hence, a single pulse would suffice for each momentum setting of the flux scan. 


\subsubsection{Polarization}

As discussed in secs. 2.4 and 3.5 and shown in Fig. 26, the polarization of positrons is to be measured by transmission polarimetry of photons obtained by (re)conversion of the positrons on a secondary target.

In a design study of the positron polarimeter [66], it was shown that its analyzing power (introduced in sec. 2.4) is insensitive to the thickness of the reconversion target, as summarized in Fig. 35. Indeed, it appears that it would suffice to use the magnetized iron itself as the reconversion target. However, a separate reconversion target, 1.75-mm of tungsten, will be used. This will permit evaluation of possible background photons that are incident on the reconversion target by short studies when the iron absorber is removed for the flux measurements (sec. 4.2.1).

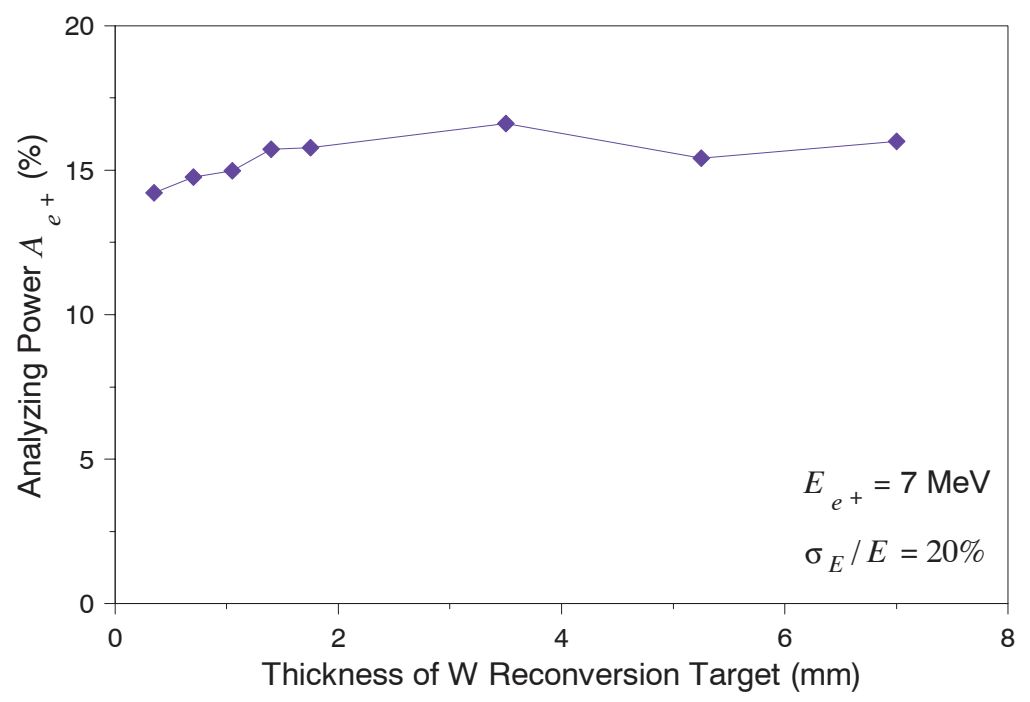

Figure 35: Simulation of the dependence of the energy-weighted analyzing power of the positron polarimeter on the thickness of the reconversion target for a 7.5-cm-long iron absorber and the polarimeter geometry shown in Fig. 26 .

The analyzing power is also insensitive to the size of the momentum acceptance of the polarimeter, as shown in Fig. 36. Therefore, the experiment can run with a relatively large acceptance of $\pm 20 \%$ around the central momentum (Fig. 27), and the rates in the positron polarimeter will be reasonably high.

The analysis of the positron polarization will consist of a scan over the central momentum of the polarimeter from 3 to $10 \mathrm{MeV}$, collecting data for about $15 \mathrm{~min}$ at each setting. Table 13 presents simulated results for such a scan. The expected positron polarization has been shown in Fig. 10. The efficiency of the positron transport from the primary target to the reconversion target is taken from Fig. 30. The expected asymmetry of reconversion photons that are detected in the CsI array on reversal of the magnetization of the iron absorber is based on the positron polarization in the second column and on an electron polarization $P_{e}$ of $7 \%$. The energy-weighted analyzing power $A_{e^{+}}$for the positron polarimeter has been 


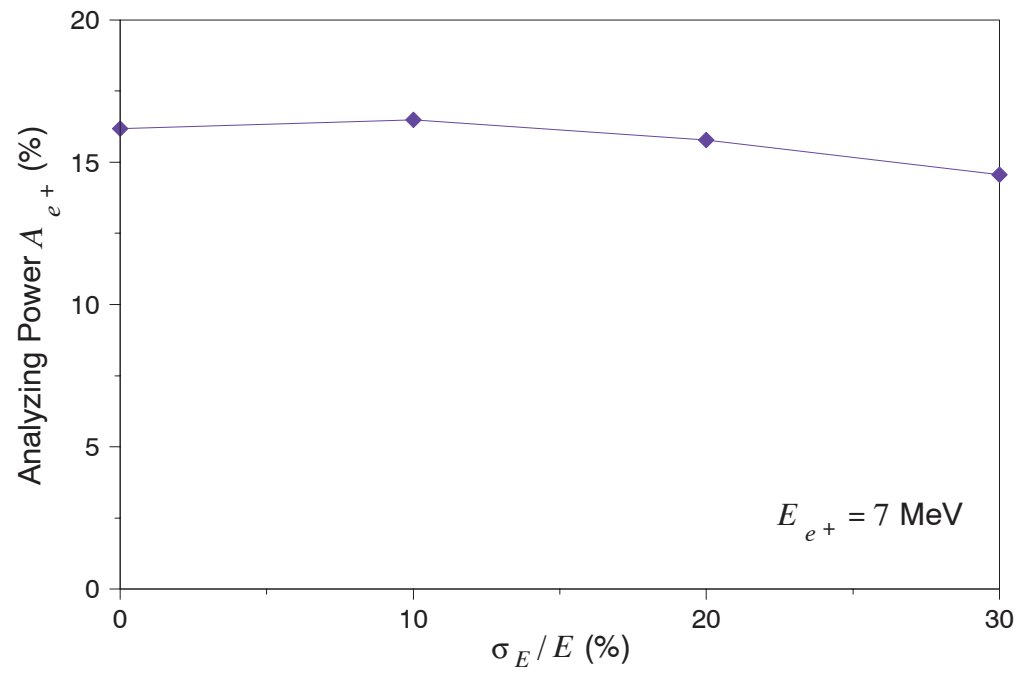

Figure 36: Simulation of the dependence of the energy-weighted analyzing power on the momentum acceptance of the positron polarimeter for a $7.5-\mathrm{cm}$ long iron absorber and the polarimeter geometry shown in Fig. 26.

presented in Fig. 17. In a 15-min run there are 27,000 pulses, for a total of $N_{e^{+}}=5.4 \times 10^{11}$ positrons produced by the undulator photons.

The positron polarization is reconstructed from the measured energy asymmetry $\delta=$ $\left(E^{+}-E^{-}\right) /\left(E^{+}+E^{-}\right)$

$$
P_{e^{+}}=\frac{\delta}{P_{e^{-}} A_{e^{+}}},
$$

where $P_{e^{-}}$is the net polarization of the electrons in the iron analyzer $\left(P_{e^{-}} \approx 0.07\right)$ and $A_{e^{+}}$is the associated energy-weighted analyzing power which must be determined from a simulation of the physical processes for the geometry of the polarimeter.

The statistical error on the measurement of the positron polarization is,

$$
\Delta P_{e^{+}}=\frac{1}{P_{e^{-}} A_{e^{+}} \sqrt{N_{\gamma}}},
$$

as summarized in the last column of Table 13, using $N_{\gamma}=\left(\epsilon_{e^{+}}\right)\left(\epsilon_{e^{+} \rightarrow \gamma}\right)\left(\epsilon_{\gamma}\right) N_{e^{+}}$, where $\epsilon_{e^{+}}$is the efficiency for transport of positrons from the primary target to the reconversion target, $\epsilon_{e^{+} \rightarrow \gamma} \approx 1$ is the reconversion efficiency and $\epsilon_{\gamma}$ is the efficiency for transport of reconversion photons to the CsI array. The statistal errors shown in last column of Table 13 correspond to the polarimeter described in Table 10, a positron intensity of $2 \times 10^{7}$ per pulse, 30 pulses per second, and a spectrometer transmission as shown in Fig. 30. This error only accounts for photon statistics and does not include calorimeter-related resolution effects. The overall error will be dominated by the systematic uncertainty in $P_{e^{-}}$as discussed earlier. 
Table 13: Simulated results of a scan of positron polarization vs. energy for the example of a 7.5-cm-long iron absorber and the polarimeter geometry shown in Fig. 26. For each positron energy, 15 min data are collected, during which $N_{e^{+}}=5.4 \times 10^{11}$ positrons are generated from undulator photons. The number of photons detected in the CsI array of the positron polarimeter is $N_{\gamma}=\left(\epsilon_{e^{+}}\right)\left(\epsilon_{e^{+} \rightarrow \gamma}\right)\left(\epsilon_{\gamma}\right) N_{e^{+}}$, with $\epsilon_{e^{+}}$and $\epsilon_{\gamma}$ as listed and $\epsilon_{e^{+} \rightarrow \gamma} \approx 1$. The 15-min run is divided into two 7.5-min segments with opposite signs of the magnetization of the iron absorber. The detected photon asymmetry $\delta$ and the associated analyzing power $A_{e^{+}}$are given for the energy-weighted signals appropriate for a calorimeter detector.

\begin{tabular}{|c|c|c|c|c|c|c|c|}
\hline $\begin{array}{c}\text { Positron } \\
\text { Energy } \\
(\mathrm{MeV}) \\
\end{array}$ & $\begin{array}{c}\text { Positron } \\
\text { Pol. } \\
P_{e^{+}}(\%)\end{array}$ & $\begin{array}{c}\text { Positron } \\
\text { Transport } \\
\text { Eff. } \epsilon_{e^{+}}(\%)\end{array}$ & $\begin{array}{c}\text { Photon } \\
\text { Transport } \\
\text { Eff. } \epsilon_{\gamma}(\%)\end{array}$ & $\begin{array}{l}\text { Photon } \\
\text { Asym. } \\
\delta(\%) \\
\end{array}$ & $\begin{array}{c}\text { Analyzing } \\
\text { Power } \\
A_{e^{+}}(\%) \\
\end{array}$ & $\begin{array}{c}N_{\gamma} \\
\text { in } 15 \mathrm{~min}\end{array}$ & $\begin{array}{c}15 \text { min } \\
\text { Abs.Ërror } \\
\Delta P_{e^{+}}(\%)\end{array}$ \\
\hline 3 & 42 & 1.5 & 0.045 & 0.55 & 18.6 & $3.7 \times 10^{6}$ & 4.0 \\
\hline 4 & 61 & 1.9 & 0.078 & 0.84 & 19.7 & $8.0 \times 10^{6}$ & 2.6 \\
\hline 5 & 69 & 2.1 & 0.12 & 0.82 & 17.0 & $1.45 \times 10^{7}$ & 2.2 \\
\hline 6 & 78 & 2.3 & 0.20 & 0.87 & 15.9 & $2.44 \times 10^{7}$ & 1.8 \\
\hline 7 & 84 & 1.7 & 0.28 & 0.93 & 15.8 & $2.59 \times 10^{7}$ & 1.6 \\
\hline 8 & 77 & 0.9 & 0.38 & 0.82 & 15.0 & $1.86 \times 10^{7}$ & 2.2 \\
\hline 9 & 64 & 0.4 & 0.50 & 0.63 & 14.0 & $1.09 \times 10^{7}$ & 3.1 \\
\hline 10 & 68 & 0.3 & 0.64 & 0.66 & 13.9 & $1.04 \times 10^{7}$ & 3.2 \\
\hline
\end{tabular}




\section{$5 \quad$ Experimental Measurements and Setup}

\subsection{Overview}

E-166 requests six weeks of beam time in the SLAC FFTB for setting up the experiment and making the required measurements. This request is based on the assumptions that the beam at the end of the linac meets the nominal beam quality requirements for the experiment and that a valid FFTB configuration exists for transport through the FFTB and onto the FFTB dump. These assumptions are reasonably met if E-166 is scheduled during the operating cycle in which the FFTB has been used for other experiments. The assumptions are notably not valid immediately after a long accelerator shutdown and during the time that PEP II is initially being brought online. Removal of the E-166 equipment has not been included in the above six weeks. A period of 2-3 days is required to remove E-166 equipment and to reinstall equipment removed for the E-166 cycle. This removal/reinstallation period can coincide with the installation of the next experiment if the scheduled activity does not occupy the same locations as the E-166 apparatus.

This six-week request is divided into a three-week block for experiment setup activities; i.e., installation, checkout, beam tuning, and initial operations followed by a three-week block for measurements. This section details the measurements that will be made in the second three week period and the setup activities in the first three week period.

\subsection{Experimental Measurements}

The E-166 beam requirements are listed in Table 14. E-166 requires a nominal single bunch beam of $50 \mathrm{GeV}$ at $30 \mathrm{~Hz}$ with a charge of $1 \times 10^{10}$ electrons per bunch, transverse rms emittance in the range of $2 \times 10^{-5} \leq \gamma \epsilon_{x}=\gamma \epsilon_{y} \leq 4 \times 10^{-5}$, and an rms energy spread $\sigma_{E} / E \leq 0.3 \%$. It is expected that the beam rate will drop to $1 \mathrm{~Hz}$ during PEP II fills, which are expected to take approximately $10 \%$ of the scheduled running time.

Table 14: E-166 beam parameter request.

\begin{tabular}{|c|c|c|c|c|c|c|}
\hline \hline$E_{e}$ & $f_{\text {rep }}$ & $N_{e}$ & $\gamma \epsilon_{x}=\gamma \epsilon_{y}$ & $\beta_{x}, \beta_{y}$ & $\sigma_{x}, \sigma_{y}$ & $\sigma_{E} / E$ \\
\hline \hline $\mathrm{GeV}$ & $\mathrm{Hz}$ & $e^{-}$ & $\mathrm{m}-\mathrm{rad}$ & $\mathrm{m}$ & $\mu \mathrm{m}$ & $\%$ \\
\hline 50 & 30 & $1 \times 10^{10}$ & $3 \times 10^{-5}$ & $5.2,5.2$ & 40 & 0.3 \\
\hline \hline
\end{tabular}

As said before, the E-166 measurements to be performed are:

- Photon flux and photon polarization will be measured at an undulator parameter of $K=0.17$.

- Positron flux and polarization will be measured at an undulator parameter of $K=$ 0.17 using a 0.5-rad. len.-thick Ti converter target with the passband of the beam spectrometer set to $7.0 \pm 1.4 \mathrm{MeV}$. The statistical error on the positron polarization 
measurement is about $3 \%$ for 15 minutes of counting with a primary beam current of $1 \times 10^{10} e^{-}$per pulse and $30-\mathrm{Hz}$ pulse rate.

- Photon flux and polarization will be measured as a function of strength parameter $K$ by varying the undulator current.

- Positron flux and polarization will be measured as a function of the central momentum of the spectrometer using an 0.5 r.l. thick pure Ti material converter target.

- Effect of target thickness will be measured with the spectrometer pass band fixed at $7.0 \pm 1.4 \mathrm{MeV}$ for Ti targets of $0.1 \mathrm{rad}$. len. and $0.25 \mathrm{rad}$. len. and for $\mathrm{W}$ targets of $0.1 \mathrm{rad}$. len., $0.25 \mathrm{rad}$. len., and $0.5 \mathrm{rad}$. len. The time to change a target sample is estimated to be about 2 hours.

- Each data set will take about 20 minutes per set, including the time to reverse magnetization in the iron absorber.

It is estimated that less than one-third of the time will be spent on photon beam measurements. The remainder of the time will be spent on positron characterization. The analysis of the results will be prompt so that potential problems can be identified and fixed.

\subsection{Experiment Setup}

E-166 will need a three-week block of FFTB beam-time for installation of equipment, its checkout on the beamline, beam tuning, and initial data taking to ensure that everything works. This initial three-week period is divided as follows:

- 1.5 weeks for installation, prebeam checkout and $50-\mathrm{GeV}$ beam tuning in the linac enclosure;

- 0.5 weeks for beam tuning in the FFTB (primarily beam size tuning at the location of the undulator and upstream collimation for background reduction);

- 0.5 weeks for equipment checkout with beam;

- 0.5 weeks for initial data taking interspersed with tuning, background reduction, and detector shielding.

At the end of this three week period E-166 will have been run as a complete system and will be ready to make measurements.

\subsubsection{Status of Required Beamline Equipment}

Figures 18 and 19 show the layout of the E-166 beamline in the FFTB enclosure in the vicinity of IP1, and $35 \mathrm{~m}$ downstream of IP1, respectively. The devices $\mathrm{BPM}_{1}, \mathrm{HSB}_{1}, \mathrm{OTR}_{\text {, }}$ Toro, and $\mathrm{PR}_{d}$ are presently installed at the desired E-166 locations, while WS, $\mathrm{BPM}_{2}, \mathrm{~A}_{u}$, the undulator, and $\mathrm{HSB}_{2}$ will require new installation (including cabling). Monitor $\mathrm{BPM}_{3}$ is presently installed but will be moved up stream by about $0.3 \mathrm{~m}$; the existing cable for 
$\mathrm{BPM}_{3}$ will reach to the new position. The dump magnet string $\mathrm{D}_{1}$ presently consists of 6 permanent magnet dipoles, of which two are presently degaussed. For 50-GeV operation, a seventh permanent dipole magnet will be added at the downstream end of the $\mathrm{D}_{1}$ string, and all seven magnets re-energized. A power supply and cabling for this is already installed in the FFTB.

The magnet $\mathrm{HSB}_{2}$ is an existing device, but not presently installed in the FFTB; cables need to be pulled and a power supply will be borrowed from the SLC North Final Focus System (NFFS). Monitor $\mathrm{BPM}_{2}$ and associated electronics modules will be borrowed from the NFFS and replaced on completion of E-166. A set of BPM cables will be pulled. Rack space and empty CAMAC slots are available in the Bldg. 407 for the needed additional power-supply and control modules.

The Short Pulse Photon Source (SPPS) diffraction grating chamber and associated vacuum windows (not shown) are presently located about $14 \mathrm{~m}$ downstream of the dump magnet string $\mathrm{D}_{1}$, and will be removed and replaced with a vacuum spool piece.

Profile monitor $\mathrm{PR}_{t}$, collimator $\mathrm{A}_{t}$, and the Target are new installations. $\mathrm{PR}_{t}$ will be borrowed from the SLAC NFFS and replaced upon completion of E-166. The target actuator is a simple in/out device. New cabling is required for these devices.

The photon polarimeter consist of a pair of aerogel Čerenkov counters, a reversible iron magnet and a silicon-tungsten calorimeter installed about two meters downstream of the polarized positron production target.

The positron spectrometer consists of two $90^{\circ}$ dipole bend magnets. These dipoles displace the produced positrons approximately $45-\mathrm{cm}$ horizontally. The positron polarimeter consists of an iron analyzer magnet and a shielded array of CsI crystals.

All these devices are new and will need power, diagnostics and readout cabling to be run from the FFTB enclosure to Bldg. 407.

Table 15 gives a status summary of the E-166 beamline equipment.

\subsubsection{Installation and Check Out Requirements}

Open access to the FFTB enclosure from the IP1 region to the downstream end of the housing is required for a period of 1.5 weeks for installation and prebeam check out of the E166 apparatus. Another 0.5 week of checkout with beam is required prior to commencement of full operations for the experiment. It is anticipated that installation of the beamline components in the IP1 and primary dumpline vicinity will be done in parallel with the installation of the target, spectrometer and polarimeters.

Five consecutive day shifts (Monday-Friday) are needed for installation and prebeam check out of beamline devices in the vicinity of IP1 and the primary beam dumpline. This is standard beamline work that will be performed by SLAC staff and includes installation, alignment, cabling, and pump out. Each new system will be tested and calibrated prior to staging for installation. Because of the 1-mm apertures of the undulator and its protection collimator, the alignment of these devices is important.

The installation and prebeam check-out of the positron target system, spectrometer and positron diagnostics as well as the photon diagnostics will be done by SLAC staff with E-166 physicists also working on swing and owl shifts to make sure that the installed devices work as designed together with the control and data-acquisition system. 
Table 15: Status of E-166 beamline equipment.

\begin{tabular}{|c|c|c|}
\hline Device & Description & Status \\
\hline $\mathrm{BPM}_{1}$ & FFTB BPM & Installed \\
\hline $\mathrm{HSB}_{1}$ & FFTB B04A & Installed \\
\hline OTR & FFTB OTR beam profile monitor & Installed \\
\hline Toro & FFTB Beam Toroid Current Monitor & Installed \\
\hline $\mathrm{BPM}_{2}$ & SLC FFS BPM & Relocate from SLAC NFFS ${ }^{\dagger}$ \\
\hline WS & FFTB Wire Scanner beam profile monitor & Needs Installation ${ }^{\dagger}$ \\
\hline $\mathrm{A}_{u}$ & Undulator protection collimator & $\mathrm{New}^{\dagger}$ \\
\hline Undulator & 1-m helical undulator & $\mathrm{New}^{\dagger}$ \\
\hline $\mathrm{BPM}_{3}$ & FFTB BPM & Installed, needs repositioning \\
\hline Hcor & FFTB horizontal corrector & Installed, needs repositioning \\
\hline $\mathrm{HSB}_{2}$ & FFTB B04B & Exists, needs reinstallation \\
\hline $\mathrm{D}_{1}$ & FFTB Dump magnet string & Needs augmentation \\
\hline $\mathrm{PR}_{d}$ & FFTB Dump Screen & Installed \\
\hline SPPS DGB & SPPS Diffraction Grating Box & Replace w/ spool \\
\hline $\mathrm{PR}_{t}$ & E-166 target screen & Relocate from SLC NFFS ${ }^{\dagger}$ \\
\hline $\mathrm{A}_{t}$ & E-166 target collimator & $\mathrm{New}^{\dagger}$ \\
\hline Target & Target actuator & $\mathrm{New}^{\dagger}$ \\
\hline $\mathrm{D}_{2}$ & E-166 $\mathrm{e}^{+}$spectrometer & $\mathrm{New}^{\dagger}$ \\
\hline$\gamma$ Diag & E-166 photon diagnostics & $\mathrm{New}^{\dagger}$ \\
\hline $\mathrm{e}^{+}$Diag & E-166 positron diagnostics & $\mathrm{New}^{\dagger}$ \\
\hline $\mathrm{e}^{-}$Dump & E-166 electron dump & $\mathrm{New}^{\dagger}$ \\
\hline
\end{tabular}

$\dagger$ Requires cabling 
Checkout with beam is required to ensure that all beamline devices are functioning as specified. This includes tests of the diagnostic devices with beam to compare with the expected performance and of the data acquisition system to ensure that the experimental data can be successfully collected. A period of 0.5 weeks is allocated for this activity. For this checkout, a 10-Hz electron beam to the FFTB dump is required; as discussed below, the E-166 undulator will not be placed in the beamline during this initial beam checkout phase.

The detectors needed for E-166 will be installed, cabled, and commissioned during the first 1.5 weeks corresponding to the prebeam installation time. These devices and detectors are associated with the photon and positron polarimetry and are listed in Table 16.

Table 16: Photon and positron polarimetry devices and detectors.

\begin{tabular}{|l|l|c|}
\hline \hline Photon Polarimetry & & \\
\hline & Incident photon flux monitor (Aerogel) & $\dagger$ \\
\hline & Iron block w/ support and mover & \\
\hline & Trans. photon flux monitor (Aerogel) & $\dagger$ \\
\hline Positron Target & $\begin{array}{l}\text { Trans. photon energy monitor } \\
\text { (Si-W Calorimeter) }\end{array}$ & $\dagger$ \\
\hline Positron Extraction Line & Dipole Pair & \\
\hline Positron Polarimetry & & \\
\hline & Positron-to-Photon converter target & \\
\hline & Iron block w/ support and mover & \\
\hline & CsI Calorimeter & \\
\hline \hline
\end{tabular}

$\dagger$ Detectors also require: HV Power Supplies; Front-end read-out electronics (with LV power); and Cabling from FFTB housing to Data Acquisition electronics in neighboring building.

The detectors will be assembled by E-166 collaborators and bench tested with their associated electronics. Tables and supports for these detectors on will be procured by SLAC and can be installed ahead of the six weeks requested for E-166.

The mechanical installation is expected to be rather straightforward; however the physical space available must be managed very carefully due to the limited space in the FFTB tunnel. After the mechanical installation, a mechanical survey of the beamline components and E-166 apparatus will be done.

Cables for power, control and data acquisition for the experiment must be pulled from Bldg. 407 into the FFTB enclosure. Most of this can be accomplished well before E-166 beam 
time. After the detectors and their associated electronics have been cabled up, connected with the appropriate power supplies $(\mathrm{HV} / \mathrm{LV})$, and linked to the readout electronics and the data-acquisition system in Bldg. 407, about 3 shifts without beam will be needed for the integration of the detectors into the data-acquisition (DAQ) system, and at least one shift with beam to commission them. This task is simplified by the fact that the detectors will have been tested on a similar DAQ system (currently LabView) and various settings such as HV for PMT's and depletion voltages for silicon detectors will have been determined before installation.

Table 17 summarizes the installation and test plan for the detectors.

Table 17: Detector installation and test plan. Some tests might be performed by physicists during the weekend preceding beam delivery.

\begin{tabular}{|l|l|}
\hline \hline Day & Activity \\
\hline \hline 1 & Install supports \\
\hline $2-4$ & Install detectors, iron blocks, cables, alignment survey \\
\hline $4-5$ & Connect detectors to HV/LV and DAQ \\
\hline $5-8$ & Test electronic readouts, DAQ system \\
\hline \hline
\end{tabular}

A further 1-2 shifts is required for experiment commissioning with beam, for the final setting of trigger times, and possible last fixes of DAQ software and repair of any bad hardware and dead read-out channels. 


\subsubsection{Beam Tuning and System Integration}

Prior to E-166 running, the linac modulators, klystrons, and SLED systems will be prepared for $50-\mathrm{GeV}$ operation at $30 \mathrm{~Hz}$. When the FFTB is in Controlled Access, the full energy linac beam is parked on 52SL1 and 52SL2 using dipole 50B1; screen PR55 allows observation of the energy-resolved beam. This allows for linac tuning for $50-\mathrm{GeV}$ operation to the beam switchyard during the 1.5 weeks of installation activities. The major steps in beam tuning for E-166 through to the FFTB dump are listed below:

- Starting with a low emittance $30 \mathrm{GeV}$ beam at end of linac, raise the linac energy to $50 \mathrm{GeV}$; steer, tune, and save configurations (done during the E-166 installation period).

- Re-establish 50-GeV beam from switchyard through to the FFTB dump. Start with the last known good FFTB configuration scaled to $50 \mathrm{GeV}$ and the last saved FFTB "gold" BPM orbit. With undulator removed, and a nominal 2-cm-I.D. beam pipe installed, steer beam to FFTB dump. Less than one shift will be needed to re-establish $50-\mathrm{GeV}$ beam from switchyard through to the FFTB dump.

- Spend one shift checking diagnostics with beam (everything should work as per prebeam checkout.)

- Tune the nominal 40- $\mu \mathrm{m}$ rms spot size by loading in the DIMAD configuration and focusing on the wire scanner before the undulator. Once the beam has been focused onto the wire scanner, the beam waist can be shifted downstream by about $1 \mathrm{~m}$ to the center of the undulator using standard operating procedures. The estimated tuneup time after nominal beam is on dump is expected to be six shifts.

- Once tuned, save reference orbits for steering feedbacks, save gold orbit, magnet settings.

- Shut off beam and install undulator. This is expected to take one shift.

- Bring back beams at low rate until full transmission through the undulator is achieved.

- Increase repetition rate to $10 \mathrm{~Hz}$ for diagnostics.

- Run up undulator current (should have negligible effect on beam), and look for undulator photons at $\mathrm{PR}_{t}$.

Three days are required to tune $50-\mathrm{GeV}$ beam to FFTB dump, checkout diagnostics, and tune a small spot at IP1 with the undulator moved off the beamline. At this point the undulator is moved onto the beamline and E-166 is ready for commissioning the complete system. This first week of commissioning will be spent making preliminary data runs, testing equipment, and repairing and replacing equipment as necessary. 


\section{References}

[1] V.E. Balakin and A.A. Mikhailichenko, The Conversion System for Obtaining High Polarized Electrons and Positrons, Budker Institute of Nuclear Physics, Preprint BINP 79-85 (1979).

[2] K.C. Moffeit, Positron Source: First Fifty Nanoseconds, SLAC-CN-268 (May 1984); F. Bulos et al., Design of a High Yield Positron Source, SLAC-PUB-3635 (April 1985), IEEE Trans. Nucl. Sci. NS32, 1832 (1985), http://www.slac.stanford.edu/pubs/slacpubs/3000/slac-pub-3635.html

[3] The NLC Collaboration, N. Phinney ed., 2001 Report on the Next Linear Collider, SLAC-R-571 (June 2001), sec. 5.4, http://www.slac.stanford.edu/pubs/slacreports/slac-r-571.html

[4] K. Floettmann, Investigations Toward the Development of Polarized and Unpolarized High Intensity Positron Sources for Linear Colliders, Ph.D. Thesis, DESY 93-161.

[5] New Kinds of Positron Sources for Linear Colliders, Proc. Stanford Workshop (March 4-7, 1997), SLAC-R-0502

[6] T. Okugi, Y. Kurihara, M. Chiba, A. Endo, R. Hamatsu, T. Hirose, T. Kumita, T. Omori, Y. Takeuchi, and M. Yoshioka, Proposed Method to Make a Highly Polarized e+ Beam for Future Linear Colliders, Jpn.J.Appl.Phys. Vol.35 (1996) 3677.

[7] T. Omori, T. Aoki, K. Dobashi, T. Hirose, Y. Kurihara, T. Okugi, I. Sakai, A. Tsunemi, J. Urakawa, M. Washio, and K. Yokoya, Design of a Polarized Positron Source for Linear Colliders, Nucl. Instr. Meth A500, 232 (2003) and Nucl. Instr. Meth. A503, 658 (2003).

[8] TESLA Technical Design Report, Chap. 4, http://tesla.desy.de/new_pages/TDR_CD/PartII/chapter04/chapter04.pdf

[9] Y.J. Batygin and J.C. Sheppard, Start-to-End Simulation of Polarized Positron Collection in the NLC, NLC Tech Note LCC-0117 (March 2003), http://www-project.slac.stanford.edu/lc/ilc/TechNotes/LCCNotes/PDF/LCC0117\%20text.pdf

[10] P. Raimondi et al., Recent Luminosity Improvements at the SLC, 17th Intl. Conf. on High-Energy Accl (HEACC 98), Dubna, Russia, SLAC-PUB-7955 (September 1998), http://www.slac.stanford.edu/cgi-wrap/getdoc/slac-pub-7955.pdf

[11] T. Hirose et al., Polarized Positron Source for the Linear Collider, JLC, Nucl. Instr. and Meth. A455, 15 (2000); K. Dobashi et al., Design of polarized-positron generation system, ibid. A455, 32 (2000).

[12] J.A. Aguilar-Saavedra et al., [ECFA/DESY LC Physics Working Group Collaboration], TESLA Technical Design Report Part III: Physics at an $e^{+} e^{-}$Linear Collider, hepph/0106315; T. Abe et al., [American Linear Collider Working Group Collaboration], 
Linear Collider Physics Resource Book for Snowmass 2001 - Part 2: Higgs and Supersymmetry Studies, in Proc. of the APS/DPF/DPB Summer Study on the Future of Particle Physics (Snowmass 2001), ed. by N. Graf, hep-ex/0106056.

[13] G. Moortgat-Pick and H.M. Steiner, Physics Opportunities with Polarized $e^{-}$and $e^{+}$ Beams at TESLA, EPJdirect C, vol. 1, C6, 1 (2001), hep-ph/0106155; G. MoortgatPick, A Future Linear Collider with polarised beams: Searches for New Physics, hepph/0303234.

[14] C. Blochinger, H. Fraas, G. Moortgat-Pick and W. Porod, Selectron Pair Production at $e^{-} e^{-}$and $e^{+} e^{-}$Colliders with Polarized Beams, Eur. Phys. J. C 24, 297 (2002), hep-ph/0201282.

[15] B.C. Allanach et al., The Snowmass Points and Slopes: Benchmarks for SUSY Searches, in Proc. of the APS/DPF/DPB Summer Study on the Future of Particle Physics (Snowmass 2001 , ed. by N. Graf, Eur. Phys. J. C 25, 113 (2002), [eConf C010630, 125 (2001), hep-ph/0202233.

[16] M. Dima et al., Mass Determination Method for the Right and Left Selectron Above Production Threshold, Phys. Rev. D 65, 071701 (2002), hep-ex/0112017.

[17] See also the links on http://www.ippp.dur.ac.uk/ gudrid/power/.

[18] J. Fleischer, K. Kolodziej and F. Jegerlehner, Transverse versus Longitudinal Polarisation Effects in $e^{+} e^{-} \rightarrow W^{+} W^{-}$, Phys. Rev. D 49, 2174 (1994).

[19] T. G. Rizzo, Transverse Polarization Signatures of Extra Dimensions at Linear Colliders, JHEP 0302, 008 (2003), hep-ph/0211374.

[20] R. Hawkings and K. Mönig, Electroweak and CP Violation Physics at a Linear Collider Z-factory, EPJdirect C, Vol. 1, C8, 1 (1999); K. Mönig, LC-PHSM-2000-059; J. Erler, S. Heinemeyer, W. Hollik, G. Weiglein and P. M. Zerwas, Physics Impact of GigaZ, Phys. Lett. B 486, 125 (2000), hep-ph/0005024.

[21] D.F. Alferov, Yu.A. Bashmakov and E.G. Bessonov, Generation of Circularly Polarized Electromagnetic Radiation, Sov. J. Tech. Phys. 21, 1408 (1976).

[22] B.M. Kincaid, A Short-period Helical Wiggler as an Important Source of Synchrotron Radiation, J. Appl. Phys. 48, 2684 (1977).

[23] J.P. Blewett and R. Chasman, Orbits and Fields in the Helical Wiggler, J. Appl. Phys. 48, 2962 (1977).

[24] A.A. Mikhailichenko, Pulsed Helical Undulator for Test at SLAC. The Polarized Production Scheme, Cornell CBN 02-10, SLAC LCC106 (Sept. 16, 2002), http://www-project.slac.stanford.edu/lc/ilc/TechNotes/LCCNotes/PDF/LCC-0106

[25] J.C. Sheppard, Helical Undulator Radiation, SLAC LCC-0095 (July 2002), http://www-project.slac.stanford.edu/lc/ilc/TechNotes/LCCNotes/PDF/LCC-0095 
[26] G.Ya. Kezerashvili et al., Collding Beam Polarization Measurement Using Superconducting Helical Undulator at the VEPP-2M Storage Ring, Nucl. Instr. and Meth. A314, 15 (1992).

[27] R. Carr et al., Performance of the Elliptically Polarizing Undulator on SPEAR, SLACPUB-6627 (Oct. 1995), Rev. Sci. Instrum. 66, 1862 (1995), http://www.slac.stanford.edu/pubs/slacpubs/6000/slac-pub-6627.html

[28] H. Olsen and L.C. Maximon, Photon and Electron Polarization in High-Energy Bremsstrahlung and Pair Production with Screening, Phys. Rev. 114, 887 (1959).

[29] J.C. Liu et al., Polarization Study for NLC Positron Source Using EGS4, SLAC-PUB8477 (June 16, 2000), http://www.slac.stanford.edu/cgi-wrap/getdoc/slac-pub-8477.pdf

[30] W.R. Nelson, H. Hirayama and D.W.O. Rogers, The EGS4 Code System, SLAC-Report265, (1985).

[31] H. Hoogduin, Electron, Positron and Photon Polarimetry, Ph.D. Thesis, University of Groningen, ISBN: 90-367-0737-4 (1997).

[32] F.W. Lipps and H.A. Tolhoek, Polarization Phenoma of Electrons and Photons. I. General Method and Application to Compton Scattering, Physica 20, 85 (1954); Polarization Phenoma of Electrons and Photons. II. Results for Compton Scattering, Physica 20, 395 (1954).

[33] L.W. Fagg and S.W. Hanna, Polarization Measurements on Nuclear Gamma Rays, Rev. Mod. Phys. 31, 711 (1959).

[34] H. Frauenfelder and A. Rossi, Determination of the Polarization of Electrons and Photons, in Methods of Experimental Physics. Nucl. Physics, Part B5, ed. by L.C.L. Yuan and C.-S. Wu (Academic Press, New York, 1963), pp. 214-274.

[35] J.C. Wheatley et al., Circular Polarization of Gamma Radiation Emitted by Oriented Co-40 Nuclei, Physica 21, 841 (1955).

[36] S.B. Gunst and L.A. Page, Compton Scattering of $2.62 \mathrm{MeV}$ Gamma Rays by Polarized Electrons, Phys. Rev. 92, 970 (1953).

[37] H. Schopper, Measurement of Circular Polarization of $\gamma$-rays, Nucl. Instr. and Meth. 3, 158 (1958), http://www-project.slac.stanford.edu/lc/local/PolarizedPositrons/doc/ClassicalPapers/ Schopper.pdf

[38] M. Fukuda et al., Polarimetry of Short Pulse Gamma-Rays and Positrons, in Proc. 2nd Asian Part. Accel. Conf. (Beijing, China, 2001), p. 881; 
M. Fukuda, T. Aoki, K. Dobashi, T. Hirose, T. Iimura, Y. Kurihara, T. Okugi, T. Omori, I. Sakai, J. Urakawa, and M. Washio, Polarimetry of Short-Pulse Gamma-Rays Produced through Inverse Compton Scattering of Circularly Polarized Laser Beams, KEK Preprint 2003-05, submitted to Phys. Rev. Lett.

[39] XCOM: Photon Cross Sections Database (Nat. Inst. Standards), http://physics.nist.gov/PhysRefData/Xcom/Text/XCOM.html

[40] L.A. Page, Polarization Effects in the Two-Quantum Annihilation of Positrons, Phys. Rev. 106, 394 (1957).

[41] William H. McMaster, Matrix Representation of Polarization, Rev. Mod. Phys. 33, 8 (1961).

[42] A. Buhler et al., A Measurement of the $e^{+}$Polarization in Muon Decay: The $e^{+}$Annihilation Method, Phys. Lett. 7, 368 (1963).

[43] A.M. Bincer, Scattering of Longitudinally Polarized Fermions, Phys. Rev. 107, 1344 (1957).

[44] G.W. Ford and C.J. Mullin, Scattering of Polarized Dirac Particles on Electrons, Phys. Rev. 108, 477 (1957).

[45] J.D. Ullmann, H. Frauenfelder, H.J. Lipkin, and A. Rossi, Determination of Electron and Positron Helicity with Møller and Bhabha Scattering, Phys. Rev. 122 (1961) 536. http://prola.aps.org/pdf/PR/v122/i2/p536_1

[46] J. Duclos et al., Measurement of the $e^{+}$Polarization in Muon Decay by Means of Bhabha Scattering, Phys. Lett. 9, 62 (1964).

[47] F. Corriveau et al., Measurement of the Positron Longitudinal Polarization in Muon Decay, Phys. Rev. D 24, 2004 (1981).

[48] R.L. Gluckstern, M.H. Hull, Jr., and G. Breit, Polarization of Bremsstrahlung Radiation, Phys. Rev. 90, 1026 (1953).

[49] C. Fronsdahl and H. Überall, Polarization of Bremsstrahlung from Polarized Electrons, Phys. Rev. 111, 580 (1958); Nuovo Cim. 8, 163 (1958).

[50] G. Culligan et al., Nature 180, 751 (1957).

[51] M. Goldhaber, L. Grodzins, and A.W. Sunyar, Evidence for Circular Polarization of Bremsstrahlung Produced by Beta Rays, Phys. Rev. 106, 826 (1957).

[52] P.C. Macq, K.M. Crowe and R.P. Haddock, Helicity of the Electron and Positron in Muon Decay, Phys. Rev. 112, 2061 (1958).

[53] S. Bloom et al., Measurement, by Bremsstrahlung Transmission, of the Polarization of $e^{+}$from $\mu^{+} \rightarrow e^{+}+\nu_{e}+\bar{\nu}_{\mu}$, Phys. Lett. 8, 87 (1964). 
[54] T. Zwart et al., Transmission Polarimetry at MIT Bates: Towards an Absolute Polarimeter?, http://mitbates.mit.edu/pesp2002/talks/ZwartXPO-PESP2002.pdf

[55] T. Hirose, Development of Picosecond CO2 Laser for Production of Polarized Positrons at Linear Colliders (April 30, 2003), http://heal1.phys.metro-u.ac.jp/ kumita/US-Japan/progress-final.pdf

[56] S.M. Harris and R.J. Jabbur, On Depolarization of Fast Positrons by Bremsstrahlung, Nuovo Cimento 32, 258 (1964).

[57] C. Bouchiat and J.M. Levy-Leblond, Depolarization of Electrons in Matter, Nuovo Cimento 33, 193 (1964).

[58] W. Chinowsky, D. Cutts, and R. Stiening, Nuovo Cimento 34, 1431 (1964).

[59] Y. Batygin, Particle-in-cell code BEAMPATH for Beam Dynamics Simulations with Space Charge, ISSN 1344-3877, RIKEN AF-AC-17 (2000).

[60] C. Bamber et al., Studies of Nonlinear QED in Collisions of $46.6 \mathrm{GeV}$ Electrons with Intense Laser Pulses, Phys. Rev. D 60, 092004 (1999).

[61] A. Mikhailichenko, Pulsed Undulator for Test at SLAC of Polarized Positron Production, CBN-03-05 (April 10, 2003), http://puhep1.princeton.edu/ ${ }^{\sim}$ mcdonald/e166/CBN 03-5.pdf

[62] MERMAID/3D, SIM Inc., Novosibirsk, P.O. Box 402, Russia.

[63] G.G. Scott, Review of Gyromagnetic Ratio Experiments, Rev. Mod. Phys. 34, 102 (1962).

[64] B. Aubert et al., The BaBar Detector, Nucl. Instr. and Meth. A479 1, (2002).

[65] M. Woods et al., Design Studies for Flux and Polarization Measurements of Photons and Positrons for SLAC Proposal E166, SLAC LCC-0107 (Nov. 6, 2002), http://www-project.slac.stanford.edu/lc/ilc/TechNotes/LCCNotes/PDF/LCC0107\%20text.pdf

[66] V. Gharibyan and K. P. Schüler, The Positron Polarimeter for the SLAC FFBT Undulator Experiment (May 2003), http://www-project.slac.stanford.edu/lc/local/PolarizedPositrons/internal/doc/2003/200305-12-ps-pospol.ps.gz 